Linköping Studies in Science and Technology

Dissertation No. 2187

Real-time multi-exposure laser speckle contrast imaging of skin microcirculatory perfusion

Martin Hultman
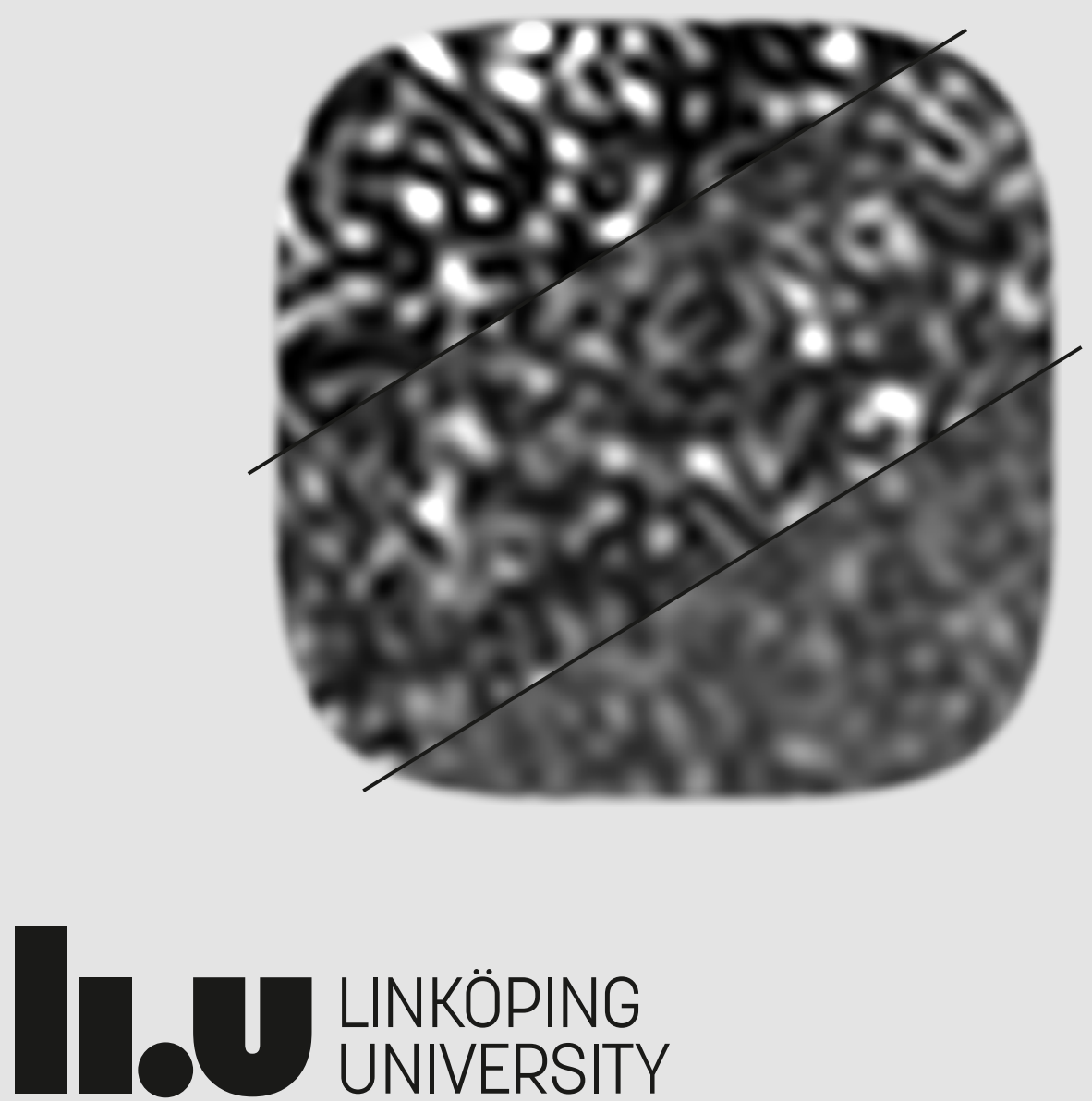

Linköping Studies in Science and Technology

Dissertations, No. 2187

\title{
Real-time multi-exposure laser speckle contrast imaging of skin microcirculatory perfusion
}

\author{
Martin Hultman
}

11.0U UNKKDPNG

Linköping University

Department of Biomedical Engineering

SE-581 83 Linköping, Sweden

Linköping 2021 

International License.

https://creativecommons.org/licenses/by/4.0/

Edition 1.0

(C) Martin Hultman, 2021

ISBN 978-91-7929-105-1 (print)

ISBN 978-91-7929-106-8 (PDF)

ISSN 0345-7524

URL https://doi.org/10.3384/9789179291068

Published articles have been reprinted with permission from the respective copyright holder.

Typeset using $\mathrm{X}_{4} \mathrm{~T}_{\mathrm{E}} \mathrm{X}$

Printed by LiU-Tryck, Linköping 2021 


\section{POPULÄRVETENSKAPLIG SAMMANFATTNING}

Mikrocirkulationen är ett samlingsbegrepp för blodflödet i kroppens minsta kärl: kapillärer, venuler, och arterioler. Mikrocirkulationen är mycket viktig eftersom den reglerar tillförseln av syrerikt och näringsrikt blod till de kringliggande cellerna. Utan en fungerande mikrocirkulation kan cellerna inte leva. Vissa sjukdomstillstånd, exempelvis diabetes eller perifer artärsjukdom, kan leda till en försämring i mikrocirkulationens förmåga att förse cellerna med den nödvändiga mängden syre och näringsämnen, vilket kan leda till vilovärk, svårläkta sår, och i värsta fall amputering eller död. Tekniker för att på ett snabbt och säkert sätt kunna utvärdera mikrocirkulationens funktion har stor potential att förbättra vården för dessa patienter.

En av dessa tekniker är så kallad laser-speckel-kontrast-avbildning (eng. laser speckle contrast imaging, LSCI) där mikrocirkulatorisk perfusion kan mätas med en kamera genom att belysa vävnaden med laserljus. Perfusion är ett mått som beror på både mängden blod i vävnaden och på blodets hastighet, och är en viktig parameter att mäta för att undersöka mikrocirkulationens funktion. När laserljus belyser en vävnad, till exempel huden, skapas ett mönster av ljusa fläckar, så kallade speckel, som rör på sig till följd av blodets rörelse i vävnaden. Genom att analysera rörelsen i bilden kan man därför beräkna vävnadens perfusion. Sedan LSCI uppfanns i början av 1980-talet har ett av de största hindren i tekniken varit en begränsning till relativa mätningar i okända enheter. Med andra ord går det att mäta hur mycket perfusionen förändras i en och samma mätning, men det är mycket svårare att jämföra mätningar mellan olika personer, olika mättillfällen, eller olika vävnader. Det här gör det svårt att dra slutsatser från LSCI och tekniken har därför inte blivit rutin i många kliniska applikationer, trots lovande forskningsresultat inom många fält. I början av 2010-talet presenterades en variant av LSCI kallad multi-exponerings-LSCI (eng. multiexposure LSCI, MELSCI). Genom att ta flera bilder av vävnaden med olika exponeringstid återfås mer information om blodets rörelse, vilket leder till bättre modeller och därmed mer korrekta perfusionsmått. Nackdelen med MELSCI är att det är en mycket långsammare metod än LSCI på grund av den stora datamängd och de komplicerade algoritmer som krävs för att göra den möjlig. Det har lett till att MELSCI, trots möjligheten till bättre perfusionsmått, inte heller har fått kliniskt genomslag.

Den här avhandlingen presenterar ett nytt system för MELSCI, som med hjälp av parallella hårdvarunära algoritmer implementerade i så kallad FPGA (eng. field-programmable gate array) kan utföra de nödvändiga algoritmerna i realtid. Nya modeller för att beräkna perfusion har också utvecklats, specifikt genom att använda maskininlärning. De nya måtten är mer verklighetsnära och mindre känsliga för brus än tidigare modeller, och kan också beräknas i realtid. Det här ger helt nya möjligheter att följa långa dynamiska förlopp i mikrocirkulationen, vilket också utvärderas i en klinisk studie där patienter behandlas för kritisk underbens-ischemi.

Utvecklingen av MELSCI som presenteras i den här avhandlingen har en stor potential att göra tekniken mer användbar i kliniska applikationer, och att göra resultaten mer lättolkade och jämförbara. Det här kan i framtiden leda till nya och förbättrade undersökningar och behandlingar för patienter med en icke-fungerande mikrocirkulation. 


\begin{abstract}
The microcirculation, the blood flow in the smallest blood vessels in the body, has a vital function as this is where oxygen and nutrients diffuses from the blood to to the surrounding cells. An important quantity is the tissue perfusion, a measure of the microcirculation's capacity to provide oxygen and nutrients to the cells. Laser speckle contrast imaging (LSCI) is a non-invasive optical technique that captures images of the microcirculatory perfusion by analysing the local contrast in the laser speckle pattern that forms when tissue is illuminated by a laser. LSCI has seen extensive use in clinical research due to the easy and cheap measurement setup, and high spatial and temporal resolution. Despite this, clinical acceptance and routine use remains low. Some of the drawbacks of the technique is a limitation to relative measurements in arbitrary units, as well as high susceptibility to measurement noise and confounding properties of the tissue. This makes comparisons difficult, especially between patients. An extension of LSCI called multi-exposure laser speckle contrast imaging (MELSCI) was proposed to deal with some of these issues, although the more complicated data acquisition and models prevented real-time use. MELSCI has instead been used exclusively as an offline technique where data is post-processed, and the clinical use has been non-existent. Furthermore, existing models for LSCI and MELSCI are designed for tissues where individual vessels are visible, such as the surface of the brain or on the retina. For measurements in the diffuse regime, such as on skin tissue, these models are no longer physiologically accurate, resulting in incorrect perfusion estimates.
\end{abstract}

This thesis presents a MELSCI-based perfusion imaging instrument that is simultaneously fast and physiologically accurate for measurements of skin. There are three main parts to this work; development of a real-time MELSCI system, development of perfusion models for skin, and demonstration of the system in a clinical feasibility study.

A real-time MELSCI instrument was developed based on a high-speed CMOS camera tightly integrated with algorithms in a field programmable gate array (FPGA). The algorithm was based on synthetic multi-exposure, where a set of 64 individual 1-ms images were digitally added to create multi-exposure images at $1,2,4,8,16,32$, and $64 \mathrm{~ms}$. The resulting multiexposure data was demonstrated to have high quality and less susceptibility to measurement noise than previous models. The instrument enabled continuous acquisition and analysis of MELSCI data in real-time at 15.6 frames per second, sufficiently fast to capture the temporal dynamics of the skin perfusion.

To enable real-time estimation of accurate and physiologically relevant perfusion from the MELSCI data, two artificial neural networks were trained on synthetic data from a mathematical model of skin. The first estimated perfusion as computed by conventional laser Doppler flowmetry (LDF), demonstrating a high correlation between the two methods. The second estimated true perfusion in absolute units $\% \mathrm{RBC} \times \mathrm{mm} / \mathrm{s}$ separated into three distinct speed components, $0-1 \mathrm{~mm} / \mathrm{s}, 1-10 \mathrm{~mm} / \mathrm{s}$ and $>10 \mathrm{~mm} / \mathrm{s}$. The ANNs removed the need for iterative optimization algorithms, resulting in more than 1000x speed-up over previous methods, and enabled real-time use in an imaging setting.

The instrument was demonstrated in controlled experiments on healthy volunteers, using standardized occlusion-release provocations, and in a clinical feasibility study where the foot perfusion was monitored during endovascular interventions in patients with chronic limb-threatening ischemia. The instrument enabled continuous imaging of perfusion, with sufficiently high framerate to capture the pulsatile dynamics, or lack thereof, at each point in time. The necessity for both high spatial and temporal resolution to properly asses the microcirculation was demonstrated.

The advancements to MELSCI proposed in this thesis has the potential to improve the clinical viability of the technique, increase interpretability of the results, and might lead to improved treatments based on a better understanding of the complex processes in the microcirculation. 


\section{Acknowledgments}

This project has simultaneously been the most difficult and most rewarding challenge I have overcome. I suppose that is nothing unusual when starting out as a researcher, but that doesn't make it any less true for me. I have always enjoyed learning new things, and I have had many opportunities to do so in this project. Both about the things I was already interested in, but also about completely new topics that I, five years ago, never knew I would be working with. I am proud of what I have accomplished in this thesis, and I hope that it is only my first step on a much longer journey.

First, I want to give my thanks to Ingemar, Marcus, and Tomas, my fantastic supervisors. For giving me the opportunity to work in such a diverse project, for guiding the way when I have needed it, but also for letting me make my own path at times. You have been, and will continue to be, great sources of inspiration with your knowledge and experience.

I also want to give a special thanks to professor Atila Alvandpour, for your contributions in the beginning of the project and before that, in my master thesis, where this journey began.

I have been fortunate to meet and work with many people in this project, and I want to thank you for your time and valuable help. To my colleagues at the hospital, thank you for guiding me into a world I was entirely unfamiliar with when I first began. I also want to thank the people at Perimed for making this project possible, and I am excited to see where it leads in the future.

Many thanks goes to all the colleagues and friends I have made at IMT. I think that you can only enjoy your work if you feel like you belong there, and I have felt that thanks to you all. I miss the fun times we used to have, back when the world was normal, and I hope that we can soon have that again.

Finally, I want to thank my parents and sister for believing in me, for supporting and encouraging me, and for putting up with all my long expositions about my exciting work. Mamma, Pappa, Johanna. I know I can always count on you to be there. Thank you, and know that I love you dearly.

This project has been financially supported by the Swedish Research Council (grant no. 2014-6141), and Sweden's Innovation Agency Vinnova via the programs MedTech4Health (Grant No. 2016-02211) and Swelife and MedTech4Health (Grant Nos. 2017-01435 and 2019-01522). 



\section{Contents}

$\begin{array}{ll}\text { Abstract } & \text { iii }\end{array}$

Acknowledgments $\quad$ v

Contents vii

List of Papers $\quad$ ix

List of Figures

1 Introduction $\quad 1$

1.1 A short history of speckle-based imaging techniques . . . . . . . 2

1.2 Medical applications . . . . . . . . . . . . . . . . 4

$\begin{array}{llr}2 & \operatorname{Aim} & 7\end{array}$

3 Laser speckles, contrast, and perfusion 9

3.1 Laser speckles . . . . . . . . . . . . . . . . . . . . . . . 9

3.2 Laser Doppler flowmetry - LDF . . . . . . . . . . . . . . . . . . . . . . . . . . . 12

3.3 Speckle contrast . . . . . . . . . . . . . . . . . 13

3.4 Single-exposure laser speckle contrast imaging - LSCI . . . . . . 16

3.5 Multi-exposure laser speckle contrast imaging - MELSCI . . . . 20

4 Computational model of skin $\quad 27$

4.1 Multi-layered skin model . . . . . . . . . . . . . . . . 28

4.2 Overview of the computational model . . . . . . . . . . . 31

4.3 Path-length distributions and measured intensity . . . . . . . . . 32

4.4 Computing the Doppler spectrum . . . . . . . . . . . . 33

4.5 Residual Doppler spectrum . . . . . . . . . . . . . . . . . . . . . . . . . . . 36

4.6 Speed-resolved perfusion . . . . . . . . . . . . . . . 37

4.7 Solving the inverse problem . . . . . . . . . 38

5 Design and implementation of a perfusion imaging instrument 41

5.1 System overview . . . . . . . . . . . . . . . 4 41 
5.2 Synthetic exposure time algorithm . . . . . . . . . . . 43

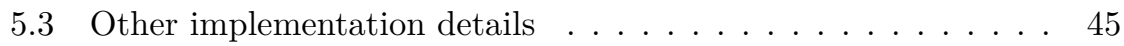

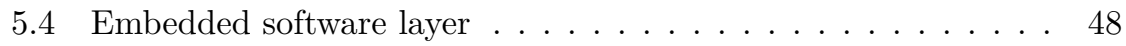

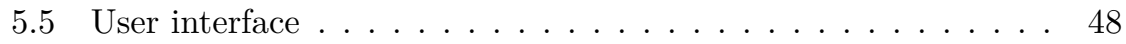

6 Review of papers $\quad 51$

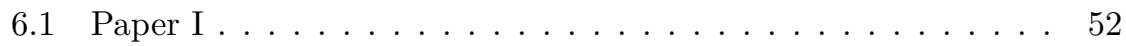

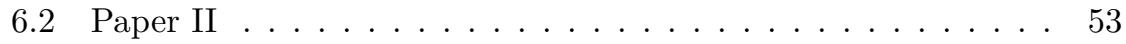

6.3 Paper III . . . . . . . . . . . . . . . . . . . . . 55

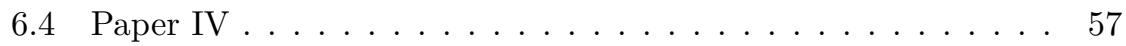

6.5 Paper V . . . . . . . . . . . . . . . . . 60

6.6 Paper VI . . . . . . . . . . . . . . . . . . . 62

7 Discussion $\quad 65$

7.1 Clinical potential . . . . . . . . . . . . . . 65

7.2 Strengths and limitations of the FPGA-based implementation . 67

7.3 Interpretability of artificial neural networks . . . . . . . . . 69

7.4 Assumptions in conventional contrast models . . . . . . . . . 70

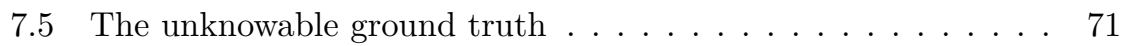

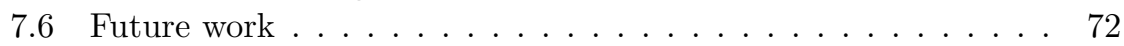

7.7 Concluding remarks $\ldots \ldots \ldots \ldots \ldots \ldots \ldots$

$\begin{array}{ll}\text { Bibliography } & 75\end{array}$

$\begin{array}{lr}\text { Paper I } & 89\end{array}$

$\begin{array}{ll}\text { Paper II } & 101\end{array}$

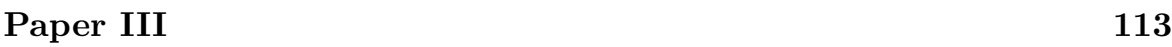

$\begin{array}{ll}\text { Paper IV } & 127\end{array}$

$\begin{array}{ll}\text { Paper V } & 145\end{array}$

$\begin{array}{ll}\text { Paper VI } & 163\end{array}$ 


\section{List of Papers}

This thesis is based on the following six publications, referred to in the text with their roman numeral:

I. M. Hultman, I. Fredriksson, M. Larsson, A. Alvandpour, T. Strömberg A 15.6 frames per second 1-megapixel multiple exposure laser speckle contrast imaging setup Journal of Biophotonics, Volume 11, Issue 2 DOI: 10.1002/jbio.201700069, LiU repository number: 1144580

II. M. Hultman, I. Fredriksson, T. Strömberg, M. Larsson Evaluation of a high framerate multi-exposure laser speckle contrast imaging setup Proceedings of SPIE, Volume 10505 DOI: $10.1117 / 12.2286248, \mathrm{LiU}$ repository number: 1221847

III. I. Fredriksson, M. Hultman, T. Strömberg, M. Larsson Machine learning in multiexposure laser speckle contrast imaging can replace conventional laser Doppler flowmetry

Journal of Biomedical Optics, Volume 24, Issue 1

DOI: 10.1117/1.JBO.24.1.016001, LiU repository number: 1315629

IV. M. Hultman, M. Larsson, T. Strömberg, I. Fredriksson

Real-time video-rate perfusion imaging using multi-exposure laser speckle contrast imaging and machine learning

Journal of Biomedical Optics, Volume 25, Issue 11

DOI: 10.1117/1.JBO.25.11.116007, LiU repository number: 1515574

V. M. Hultman, S. Aronsson, I. Fredriksson, H. Zachrisson, H. Pärsson,

M. Larsson, T. Strömberg

Comprehensive imaging of microcirculatory changes in the foot during endovascular intervention - a technical feasibility study

Submitted to Microvascular Research

VI. M. Hultman, M. Larsson, T. Strömberg, I. Fredriksson

Speed-resolved perfusion imaging using multi-exposure laser speckle contrast imaging and machine learning

In manuscript 



\section{List of Figures}

3.1 Static speckle patterns . . . . . . . . . . . . . . . . . . 10

3.2 A scattering event . . . . . . . . . . . . . . . 10

3.3 The beat effect in interfering waves . . . . . . . . . . . . . . . . . 11

3.4 Speckle movement over time . . . . . . . . . . . . . . . . . 12

3.5 Speckle patterns at different exposure times . . . . . . . . . . . . . . 14

3.6 Example of typical contrast curves . . . . . . . . . . . . . . 16

3.7 Illustration of insufficient information in single-exposure LSCI . . . . . 20

3.8 Multi-exposure acquisition schemes . . . . . . . . . . . . . . . 22

3.9 Multi-exposure noise model $1 \ldots \ldots \ldots \ldots \ldots$. . . . . . . . . . . . . . . . . . . . . . 24

3.10 Multi-exposure noise model $2 \ldots \ldots \ldots$. . . . . . . . . . 24

4.1 Influence of skin properties on contrast . . . . . . . . . . . . . 28

4.2 Distribution of skin properties in the model . . . . . . . . . . . . . . 29

4.3 Example of RBC speed distributions . . . . . . . . . . . . 30

4.4 Overview of the computational model . . . . . . . . . . . . . . 31

4.5 Example path-length distributions . . . . . . . . . . . . . . 32

4.6 Residual Doppler spectrum . . . . . . . . . . . . . . . . . . . 37

4.7 Speckle realization noise model . . . . . . . . . . . . . . . . 40

5.1 Overview of the perfusion imaging system . . . . . . . . . . . . . . . . 42

5.2 System PCB . . . . . . . . . . . . . . . . . . . . 43

5.3 Binary tree accumulation for synthetic exposure times . . . . . . . . . . 44

5.4 Ring-buffered memory . . . . . . . . . . . . . . . . . . . . . 47

5.5 User interface . . . . . . . . . . . . . . . . . . . . . . . . . . 49

6.1 Paper I - Multi-exposure contrast images . . . . . . . . . . . . . . . . 53

6.2 Paper II - Perfusion images . . . . . . . . . . . . . . . . . . 54

6.3 Paper II - MELSCI and LSCI compared to LDF . . . . . . . . . . . . . . . . 54

6.4 Paper III - Model accuracy comparison . . . . . . . . . . . . . . . . 56

6.5 Paper III - Perfusion images . . . . . . . . . . . . . . . . . . . . 57

6.6 Paper IV - Perfusion images . . . . . . . . . . . . . . . . . . . 59

6.7 Paper V - Perfusion images and graphs . . . . . . . . . . . . . . . 61

6.8 Paper V - Possible detection of micro-emboli . . . . . . . . . . . . . . . 61

6.9 Paper VI - Speed-resolved perfusion images . . . . . . . . . . . . . . . . . 63

6.10 Paper VI - Speed-resolved perfusion ROIs . . . . . . . . . . . . . . . . . . 64 



\section{1}

\section{Introduction}

The microcirculation is the common name for the complex network of arterioles, venules, and capillary blood vessels that exist in all living tissue. Perhaps the most important purpose of these vessels is to allow oxygen and nutrients carried by the blood to diffuse into the surrounding cells, and likewise for carbon dioxide and other waste products to diffuse into the blood to be removed from the tissue. Without a steady supply of oxygen and nutrients, the cells cannot function, and a working microcirculation is therefore a necessity for life. If the microcirculation becomes impaired, either due to damage or disease, the ability to investigate the extent of the impairment can be a helpful tool for diagnosis and treatment. But measuring the microcirculatory function in an accurate way, without affecting the blood flow that is measured, is not trivial. Not only is the structure of the vessel network very complex, the vessels themselves are also microscopic, several times thinner than the average human hair. To complicate the matter further, in many tissues the vessels are embedded deep in the diffusely scattering matrix of the surrounding cells, and the blood flow can therefore not be directly observed. However, one way is to use the properties of light to probe the tissue for information.

This thesis focuses on measuring microvascular perfusion, a combined measure of the concentration and speed of red blood cells (RBCs) in the capillaries, venules, and arterioles. More specifically, it is defined as concentration $\times$ speed, and is measured in the unit $\mathrm{g} \mathrm{RBC} / \mathbf{1 0 0 g}$ tissue $\times \mathbf{m m} / \mathbf{s}$, or equivalently $\% \mathbf{R B C} \times \mathbf{m m} / \mathbf{s}$, where the first part reflects the concentration of RBCs in a given volume of tissue and the second reflects the speed of the RBCs. The level of perfusion indicates the capacity to provide oxygen and nutrients to the tissue, and can be high both with high flow speeds and with high concentration of blood.

The phenomenon that allows the use of light to investigate the microcirculatory perfusion is scattering, i.e. the process where the light changes direction due to interaction with particles in the medium. In this thesis, we only consider the superficial layers of human skin, but light scattering is a universal phenomenon that is visible everywhere. The classical example is how the sky appears blue because of scattering, where blue light is scattered strongly in the atmosphere due to its short wavelength, and therefore appear 
to come from all directions. After one or more scattering events, light that is shined on skin might be backscattered out of the tissue, at which point it can be detected with a single photo-detector or camera. In particular interest for our applications is the phenomena known as the optical Doppler effect, which occurs when light scatters from a moving particle such as a red blood cell. The dynamic scattering event changes the frequency of the light. If the original light is coherent, such as a laser, the Doppler frequency shifts can be detected as tiny moving bright spots on the detector, caused by constructive and destructive interference. These are called speckles, and are the basis for the techniques use in this thesis. By analyzing the statistical properties of the speckle fluctuations, either in a single point or an image, it is possible to extract information about the blood flow in the tissue.

The first biomedical applications of this phenomenon were single-point measurements, where a high sample-rate photo-detector captured the temporal statistics of the speckles. However, the microcirculation has a highly heterogeneous structure, and a single-point measurement might not be representative of the overall tissue status. For this reason, imaging techniques based on speckles are desired. At the same time, the microcirculation is a dynamic system, and an image at a single point in time might not be an adequate representation of the tissue function. The ideal speckle measurement technique should therefore have both high spatial and temporal resolution, to capture the dynamics of the microcirculation over a wide area.

\subsection{A short history of speckle-based imaging techniques}

The first speckle-based measurement technique for in-vivo biomedical applications was presented in the 1970s by Stern et al. [1, 2]. By measuring the fluctuating speckle intensity with a single photo-detector, sampled at $20 \mathrm{kHz}$ or more, the power spectral density of the signal could be analysed to reveal information about the blood speed and concentration. Initially called laser Doppler spectroscopy, the name would eventually settle on laser Doppler flowmetry (LDF). The development continued during the 1980s, for example with work by Bonner and Nossal [3] and Nilsson [4]. LDF is a point-wise technique, but work by Tenland et al. [5] in 1983 demonstrated that the perfusion measured on different sites of the skin was highly heterogeneous. This was demonstrated again by Braverman et al. [6, 7] in the early 1990s. Using histological evaluation, different perfusion measurements were shown to represent different vessel structures, such as ascending arterioles or capillary zones, clearly demonstrating the need for imaging modalities. Even before that, in 1989, the first imaging device based on scanning LDF was proposed by Nilsson et al. [8], called laser Doppler perfusion imaging (LDPI). Although LDPI produced perfusion images, the scanning setup meant that acquisition times of several minutes were required [9]. Motion artifacts, as well as natu- 
ral variations in the tissue perfusion, therefore were confounding factors. The first LDPI system to overcome this requirement was presented in 2002 and the years following by Serov et al. [10, 11, 12], using a high-speed CMOS sensor and a full-field illumination of the tissue. While the initial instruments computed perfusion images with insufficient framerates to fully capture the dynamics of the blood flow, a more recent publication has shown promising results for this technique [13].

In parallel with the work on LDF in the late 1970s and early 1980s, the first attempts were made to use the spatial statistics of a speckle image for biomedical applications, when Fercher and Briers [14] photographed the human retina under laser illumination. After high-pass filtering, this image revealed the retinal blood vessels. They called the technique single-exposure speckle photography. Although an important achievement, the practical usefulness of the technique was impeded by the analog nature of the acquisition and processing, and it would be until 1995 before the next big development. Enabled by the development of digital cameras, Briers and Webster [15, 16] presented a fully digital variant called laser speckle contrast analysis (LASCA). By analysing the motion blur created by laser speckles as they move in the images, or more specifically the decrease in image contrast, information about the blood flow was obtained. Over the next decade, the speed of the technique progressed with developments in camera technology and faster algorithms and computers, and eventually the name laser speckle contrast imaging (LSCI) was adopted to highlight the differences from the early versions and from single-point LDF [17]. LSCI was shown to reproduce the results of LDF when imaging the cerebral blood flow in rats [18], using the same mathematical models that Fercher and Briers developed for their original application of imaging the retinal blood vessels. However, measurements on other tissues, in particular skin, is subject to more confounding factors than on the retina or the brain. In these settings, the assumptions made in conventional LSCI are no longer accurate.

To address several of the weaknesses in LSCI, Parthasarathy et al. [19] proposed multi-exposure laser speckle contrast imaging (MELSCI) in 2008. By analysing the speckle contrast at different exposure times, more information about the blood flow was obtained, and more accurate perfusion estimates were possible. Thompson et al. [20] later showed that with a sufficiently wide range of exposure times, the Doppler power spectrum could be computed from the MELSCI measurements, demonstrating that the information content in the two techniques are essentially equal. The main drawback of the proposed technique was the much slower acquisition time compared to LSCI, a consequence of the multiple exposures. In short, multi-exposure images were captured sequentially, resulting in a total acquisition time far longer than any single exposure. To address this, Dragojević et al. [21] presented synthetic MELSCI in 2015, in which the multi-exposure images were not captured by the camera, but instead computed in post-processing by adding consecutive images pixel by pixel. This technique relied on a high-speed camera to capture 
a stream of short-exposure images, and thus put much higher requirements on the data collection and processing. Prior to this thesis, only a few publications have used this method $[22,23]$, and none have achieved the high framerates and real-time performance of LSCI. Furthermore, current published work on MELSCI almost exclusively focus on cerebral blood flow, with a perfusion model modified from the original by Fercher and Briers [14], which was designed for tissues with highly visible vessels. Therefore, despite the increased information acquired in MELSCI compared to LSCI, the technique should still not be considered reliable for skin perfusion. There is a need for MELSCIbased models designed to estimate skin perfusion, to enable more accurate tissue imaging for applications such as burns and ischemic wounds. At the same time, the high spatial and temporal resolution of LSCI should preferably be retained.

\subsection{Medical applications}

Laser speckle imaging techniques are very versatile. An unobstructed view of the tissue is required, and the movement of the tissue should be minimized to reduce motion artifacts, but beyond that there are few obstacles. As such, the techniques have been used for research in many different clinical applications, often with promising results. Despite this, there are few applications in which laser speckle techniques have been accepted for routine use.

The original application of LSCI was to image the retinal blood vessels $[14,24]$. Today, ophthalmology is still the largest area of research for any speckle imaging technique [25], although the mathematical models to compute the relative flow measures are different from most other application due to historical conventions [26, 27]. Studies have, for example, investigated the changes in ocular blood flow in patients with diabetic retinopathy or glaucoma [28]. LSCI has also been combined with confocal microscopy to give a multimodal view of the retinal and choroidal circulation [29].

Another research area in which LSCI has shown potential is in neurosurgery, to monitor cerebral blood flow. As summarized by Richards et al. [30], some applications include surgical revascularization [31, 32, 33], awake functional mapping [34], brain tumor resection [35, 36], visualization of cortical spreading depolarization [37], and to predict cortical infarction during decompressive surgery for treatment of malignant stroke [38]. To the best of my knowledge, this is also the only field in which MELSCI has been used in a medical application on humans (prior to this thesis), although only in a single study [30]. The majority of the few studies with MELSCI have instead investigated the cerebral blood flow in rats or mice, either with an open cranial window [39], or through the thinned skull [40].

A commonality between measurements on the retina and on the brain is that the vessels are directly visible. This is in contrast to measurements on, 
for example, the skin, where the blood flow is only measurable through the diffusely scattering tissue. Despite this, the same techniques and mathematical models have been applied to many applications in this setting.

One such application is assessment of burn wounds and their healing potential. In a recent review article, Claes et al. [41] summarized the past and current use of LDPI in the field, concluding that the accuracy of the technique surpasses human experts. They recommended that all modern specialist burn centers should rely on speckle-based techniques for a more accurate assessment of wound depth and healing time. The use of LSCI for burns is far less common, although the same accuracy as LDPI has been demonstrated (compare [42] and [43]). Recently, it was also demonstrated that analysing the perfusion pulsatility in the burn wound greatly increased the predictive value for the healing potential [44]. This requires LSCI, since currently available LDPI-devices are scanning, and thus cannot resolve the fast pulsatile information.

In contrast to burns, where damage to the microvasculature is often severe, the microcirculation can also suffer a slow degradation from certain chronic diseases. One example is peripheral arterial disease (PAD), a progressive disorder characterized by stenosis and/or occlusion in the arteries, most often in the lower extremities [45]. In the worst cases, the disease progresses to chronic limb-threatening ischemia (CLTI), often requiring surgical intervention to avoid amputation. Diagnosis of CLTI is commonly based on macrovascular tests, such as ankle-brachial index (ABI), toe-brachial index (TBI), and Doppler ultrasound [46], but microvascular assessment might provide valuable additional information. Although LSCI has not been evaluated enough in CLTI to gain clinical acceptance, it has been demonstrated that it can be used to distinguish between ischemic and non-ischemic patients [47, 48 , and that improvements in perfusion can be detected after surgical intervention $[49,50]$. LSCI has also been used to monitor the healing progression of ischemic wounds, a common complication in patients with PAD, although no significant predictive value was found [51]. Remarkably, prior to this thesis, no studies have investigated the use of LSCI or MELSCI during CLTI intervention surgery. If the reliability of measurements in this setting could be demonstrated, it could be used to inform the interventionist about the microcirculation status in real-time, potentially improving on-table decisions.

There are many other applications, not mentioned here, in which LSCI have been applied with promising results. This small selection of applications is only meant to give a broad overview and provide context for the work that will now be presented. 


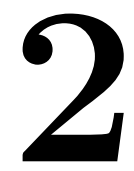

\section{Aim}

The aim of this thesis was to address several limitations of previous speckle imaging techniques - the slow computation time, the physiologically questionable assumptions, and the relative measurements in arbitrary units - in order to improve interpretability of the measurements, and to facilitate clinical use. More specifically, the aim was to:

1. Develop an instrument for acquiring and processing MELSCI data in real-time, to facilitate continuous imaging and presentation of dynamic blood flow.

2. Investigate how to compute multi-exposure contrast from Monte Carlo simulations in physiologically relevant skin models, including realistic measurement noise.

3. Develop a real-time method for estimating speed-resolved skin perfusion in absolute units $\% \mathrm{RBC} \times \mathrm{mm} / \mathrm{s}$ from multi-exposure contrast.

4. Demonstrate the technique in a clinical setting during endovascular intervention in patients with chronic limb-threatening ischemia (CLTI). 
2. AIM 


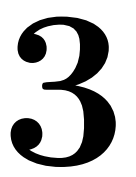

\section{Laser speckles, contrast, and perfusion}

Laser speckles is a seemingly random interference phenomenon that is present in all measurements using coherent light. For many applications it is considered a nuisance that decrease experimental precision and should be avoided or corrected for [52]. However, the speckles carry information about any medium the light has interacted with, and in the 1970s and early 1980s, Goodman developed the theory of the statistical properties of speckles [53], and Fercher and Briers showed the first biomedical imaging application based on this fact [14]. This gave birth to several new techniques where the speckles are not considered a source of noise, but instead carriers of information. Three such techniques have been used extensively in this thesis; laser speckle contrast imaging (LSCI), multi-exposure laser speckle contrast imaging (MELSCI), and laser Doppler flowmetry (LDF). These will be the focus of this chapter. But before we dive into the actual measurement techniques, we will first cover the theory behind the formation of speckles and speckle contrast, as well as some of the factors that influence these.

\subsection{Laser speckles}

The basic principle of speckle formation is relatively simple. Light that is backscattered from a rough surface or a diffuse medium will have an essentially uniform phase distribution due to the length difference in different light paths. When this light mixes on a detector, a pattern of bright and dark spots is created by the constructive and destructive interference. The bright spots are called speckles, and the interference pattern as a whole, a speckle pattern. This is illustrated in Figure 3.1.

A static, i.e. non-moving, surface or medium results in a static speckle pattern. However, if there are moving particles in the medium, light that scatters off these will change frequency due to the Doppler effect, where the change in frequency depends on the speed of the scattering particle, the direction of 

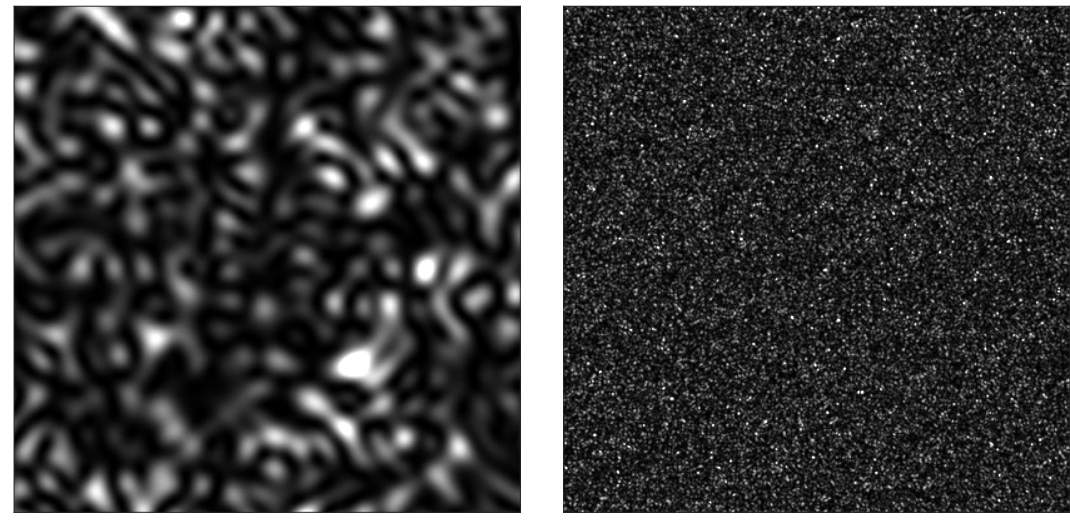

Figure 3.1: Simulation of static speckle patterns using 1000 electric field sources mixing on a $1 \times 1 \mathrm{~cm}$ detector (left) and a $10 \times 10 \mathrm{~cm}$ detector (right). The sources were distributed randomly in a $0.5 \times 0.5 \mathrm{~mm}$ square centred in relation to the detector, at $30 \mathrm{~cm}$ distance. Frequencies were centered on $381 \mathrm{THz}$, i.e. a $785 \mathrm{~nm}$ wavelength in vacuum. Phases were uniformly random from 0 to $2 \pi$.

both the particle and the light wave, and the scattering angle, according to

$$
f=\mathbf{v} \cdot \mathbf{q}=\frac{2 n v}{\lambda} \sin \left(\frac{\theta}{2}\right) \cos (\phi)
$$

where $f$ is the Doppler frequency shift, $\mathbf{v}$ is the velocity of the scattering particle, $v=|\mathbf{v}|$, and $\mathbf{q}$ is the vector indicating the change in direction of the light wave due to the scattering event $[54,55]$. The vectors and angles are illustrated in Figure 3.2.

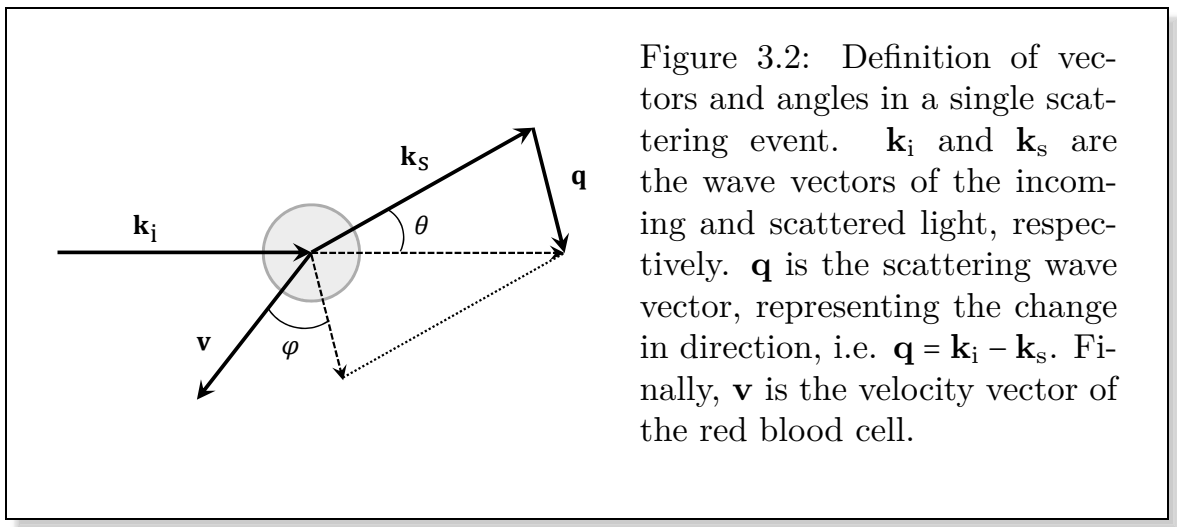


For normal flow velocities in the microcirculation, $f$ is typically on the order of kilohertz [56], an almost insignificant change for visible light which range from $750 \mathrm{THz}$ for blue light to $380 \mathrm{THz}$ in the near-infrared. Directly detecting this change by measuring the frequency of the electromagnetic field is still beyond our technological capabilities. However, when two electromagnetic fields with different frequencies interfere, the intensity of the mixed light will fluctuate with a frequency equal to the difference between the two original signals, as illustrated in Figure 3.3. This change in intensity, called the beat effect, can be detected by fairly standard photo-detectors. It is therefore possible to extract the Doppler frequency shift without directly measuring the frequency of the electric field.

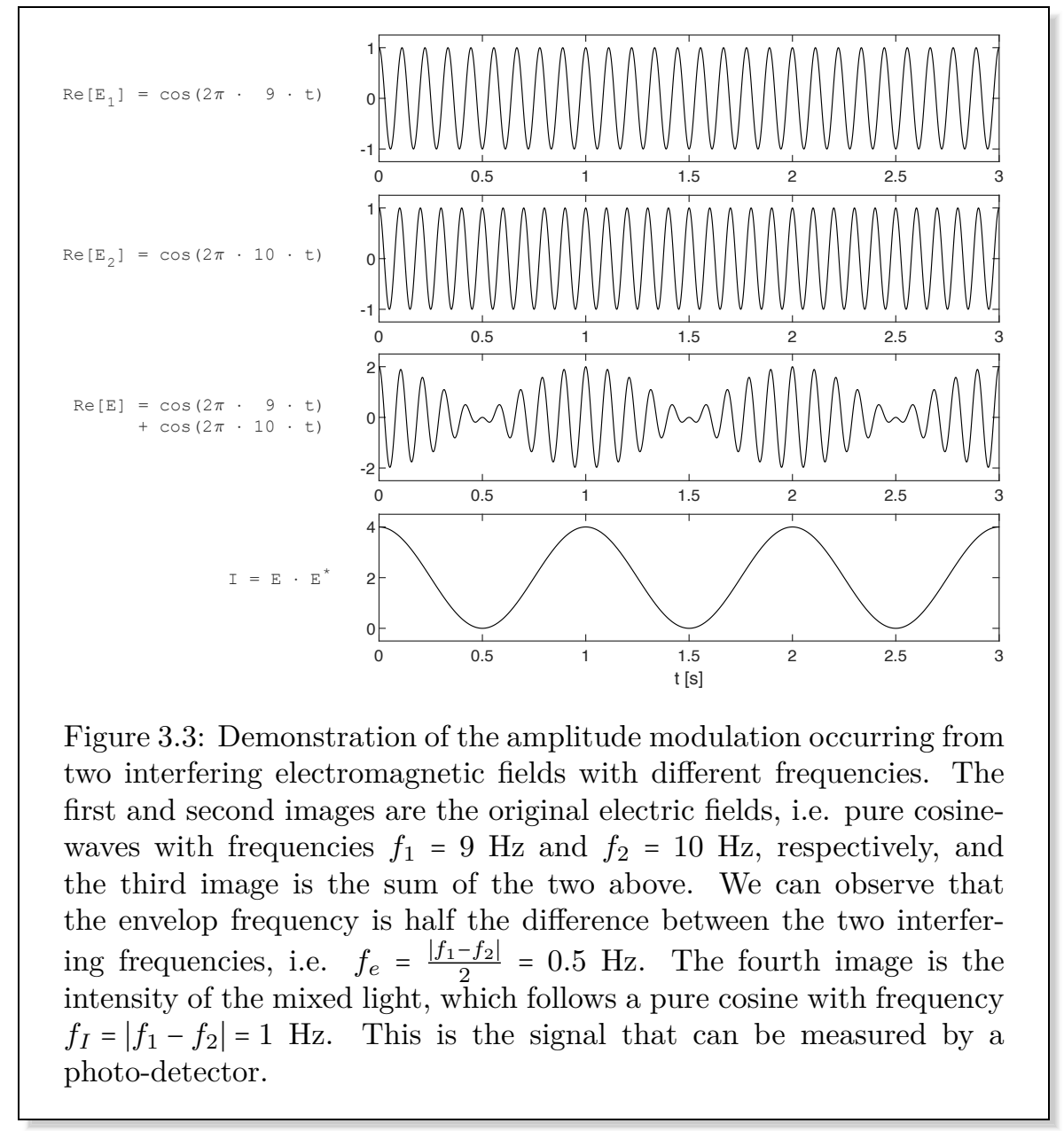

The backscattered light from a biological medium such as skin will contain a spectrum of Doppler frequency shifts. When the Doppler-shifted light 
mixes on a detector, the resulting speckle pattern will display the beat effect, although the spectrum of frequencies will make the fluctuations chaotic. These fluctuations are perceived as speckles appearing, disappearing, and moving on the detector, in seemingly random paths. This is illustrated in Figure 3.4.

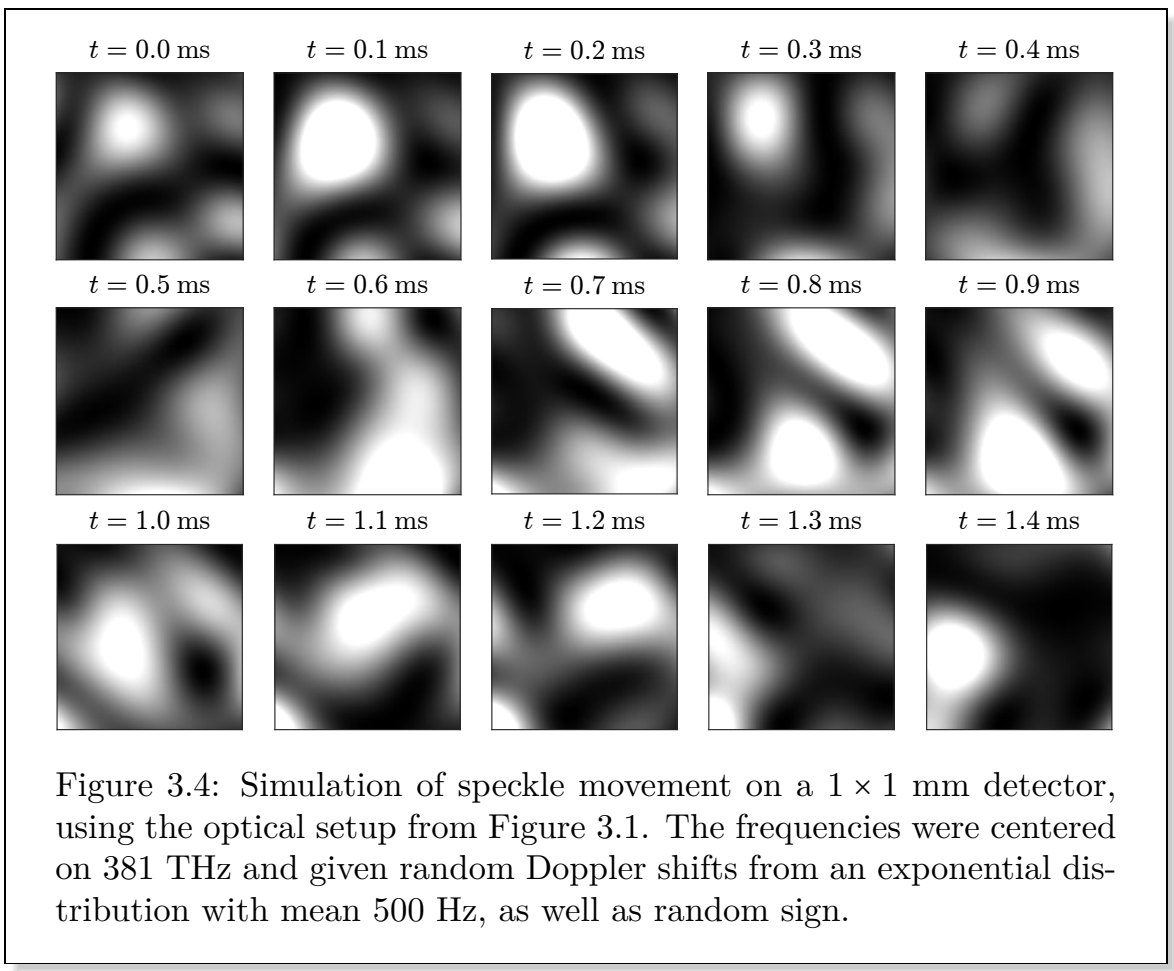

The information about the scattering particles is encoded in the movements of the speckles, and can be analysed in several ways. One is by computing the power spectrum $P(f)$ of the intensity signal, which indirectly contains the probability of Doppler frequency shifts [54]. Alternatively, it is possible to quantify the motion of the speckles using the motion blur they create when moving on the detector. The former is the principle behind LDF, and the latter behind LSCI and MELSCI. Other speckle-related techniques, such as diffuse correlation spectroscopy (DCS), will not be covered in this thesis.

\subsection{Laser Doppler flowmetry - LDF}

Laser Doppler flowmetry has not been the focus of development in this thesis. Rather, it has been used exclusively as a validated reference method to compare the perfusion estimates computed using the new methods, based 
on MELSCI. For this reason the theory behind LDF will be kept to a bare minimum in order to understand how the reference perfusion is computed.

The information about the speed of the scattering particles in tissue is encoded in the frequency content of the detected light fluctuations. In LDF, this is analysed directly by computing the power spectrum of the detected signal, usually refereed to as the Doppler power spectrum. Given a detected intensity $i=i_{\mathrm{dc}}+i_{\mathrm{ac}}$, where $i_{\mathrm{dc}}$ is the average intensity and $i_{\mathrm{ac}}$ is the dynamic component in the signal, the Doppler power spectrum is computed as

$$
P(f)=\left|\mathcal{F}\left(i_{\mathrm{ac}}\right)^{2}\right|,
$$

where $\mathcal{F}$ denotes the Fourier transform. Conventional LDF perfusion is computed as the first moment of the Doppler power spectrum:

$$
P_{\mathrm{LDF}}=\frac{1}{i_{\mathrm{dc}}^{2}} \int_{0}^{\infty} f P(f) \mathrm{d} f,
$$

where the normalization with $i_{\mathrm{dc}}^{2}$ is to make the perfusion measure independent of the signal intensity. $P_{\text {LDF }}$ scales linearly with the width of the Doppler power spectrum, which is linearly dependent on the average speed of red blood cells $\left(\left\langle v_{\mathrm{RBC}}\right\rangle\right)$, according to Equation 3.1. For low concentrations of red blood cells $\left(c_{\mathrm{RBC}}\right), P_{\mathrm{LDF}}$ is approximately linear with true perfusion $\left\langle v_{\mathrm{RBC}}\right\rangle \times c_{\mathrm{RBC}}$, although it is dependent on both the photon path-lengths and tissue scattering. At higher concentration of blood, there are further non-linear effects from $c_{\mathrm{RBC}}$, due to multiple Doppler shifts. [57]

\subsection{Speckle contrast}

The speckle patterns in Figure 3.1 and 3.4 are instantaneous realizations, i.e. the simulated detector had no exposure time. This is not how detectors work in the real world, where the photo-current instead is measured over a short, but non-zero, exposure time $T$. Since the speckles are moving on the detector, this creates motion blur in the recorded data, where longer exposure times naturally result in more motion blur. The amount of blur is also influenced by the speed of the speckles. Faster speckle movement results in blurrier images, where the speed of the speckles is determined by the distribution of Doppler shifts, which in turn is a result of the underlying movement of scatterers. There is therefore a connection between the blurriness of the speckle image and the movement of scatterers in the medium, and by analysing the blur it is possible to infer information about this movement. Specifically, speckle contrast techniques do not compute the blur, but rather the inverse of blur. As the name suggest, this is called the speckle contrast, and is defined as

$$
K=\frac{\sigma}{\langle I\rangle}=\frac{\sqrt{\left\langle I^{2}\right\rangle-\langle I\rangle^{2}}}{\langle I\rangle},
$$


where $\sigma$ is the standard deviation of the speckle intensity and $\langle I\rangle$ is the average intensity. It should be noted that $I$ in this equation is equivalent to $i$ in the case of LDF (see Section 3.2). Similarly, $\langle I\rangle$ is the same as $i_{\mathrm{dc}}$. The notation is only different by convention.

Normally the squared contrast is used in models, and further adding the fact that the contrast is dependent on the exposure time, we get the function $K^{2}(T)$, which will be commonly occurring in this chapter. Figure 3.5 illustrates the effect of different exposure times, and the associated contrast.

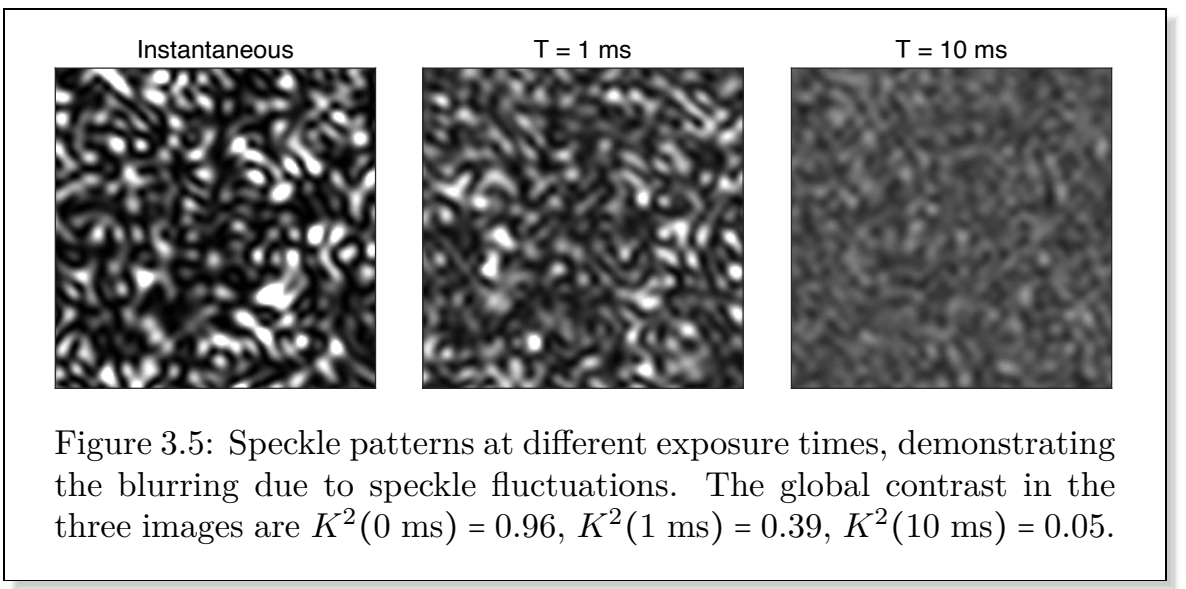

If the detected light $I$ originates from both static and moving scatterers in the medium, we can write it as a sum of two parts $I=I_{d}+I_{s}$, one dynamic and one static. We can then define the fraction of Doppler-shifted light

$$
\rho=\frac{I_{d}}{I_{d}+I_{s}}
$$

It can be shown that the contrast will approach the value $1-\rho$ when the exposure time $T$ approaches infinity [58], and by extension $K^{2}(T)$ approaches $(1-\rho)^{2}$. Complementary to this, we can intuitively understand that when the exposure time is shortened, the contrast increases since the speckles have less time to move on the detector. In the limit when $T$ approaches $0, K^{2}(T)$ approaches the value $1 / \beta \in(0,1]$. This maximum value of the contrast depends on the number of speckles per pixel, and the polarization direction of the source and detector [59]. $\beta$ can be measured experimentally, which is detailed in the next section.

Equation 3.4 describes how to compute contrast from measured speckle images. However, it is also possible to theoretically connect the Doppler frequency shifts to the contrast. The complete mathematical framework for this has been described by Fredriksson and Larsson [54], and will only be summarized here. First, given the Doppler power spectrum $P(f)$, where we 
normalize $P(0)=1$, we can compute the intensity autocorrelation function via the inverse Fourier transform:

$$
g^{(2)}(\tau)=\left|\mathcal{F}^{-1}(P(f))\right|
$$

We then use the extended Siegert relation [60] to get the electric field autocorrelation function

$$
g^{(1)}(\tau)=\sqrt{1+g^{(2)}(\tau)-g^{(2)}(0)},
$$

where the extension term $g^{(2)}(0)$ is required for light that has not been fully Doppler shifted, i.e. $\rho<1$ [61]. Another option is to compute $g^{(1)}(\tau)$ directly from the Doppler histogram $H(f)$, i.e. the probability density function of the Doppler frequency shifts. This is also sometimes called the optical Doppler spectrum, but I will use the term Doppler histogram to differentiate it from the Doppler power spectrum $P(f)$. Assuming a correctly normalized $H(f)$, such that $H(0)=1-\rho$ and the total probability over all frequencies is 1 , we again get the electric field autocorrelation function

$$
g^{(1)}(\tau)=\left|\mathcal{F}^{-1}(H(f))\right|
$$

as explained by the Weiner-Khinchin theroem [62]. Unlike $P(f), H(f)$ cannot be directly measured, but it can be obtained from the mathematical model described in Chapter 4. Regardless of whether $g^{(1)}(\tau)$ is obtained from $H(f)$ or $P(f)$, we can now express the contrast as

$$
K^{2}(T)=\frac{2 \beta}{T} \int_{0}^{T}\left|g^{(1)}(\tau)\right|^{2}\left(1-\frac{\tau}{T}\right) \mathrm{d} \tau
$$

i.e. the linearly weighted integral of the squared $g^{(1)}(\tau)$ from 0 to the exposure time $T$. The linear weighting is a correction to the equation originally proposed by Fercher and Briers [14], which has been suggested by several authors $[63,64]$. Also note the inclusion of $\beta$ in Equation 3.9, to correct for the effects of number of speckles, and polarization, as mentioned previously.

Figure 3.6 illustrates a few typical contrast decay curves, and summarizes some key parameters discussed in this section. We can observe the effect from different levels of Doppler fraction $\rho$, and different mean scatterer speeds $\left\langle v_{\mathrm{RBC}}\right\rangle$. $\beta$ is set to 1 , emulating a perfectly calibrated measurement. 

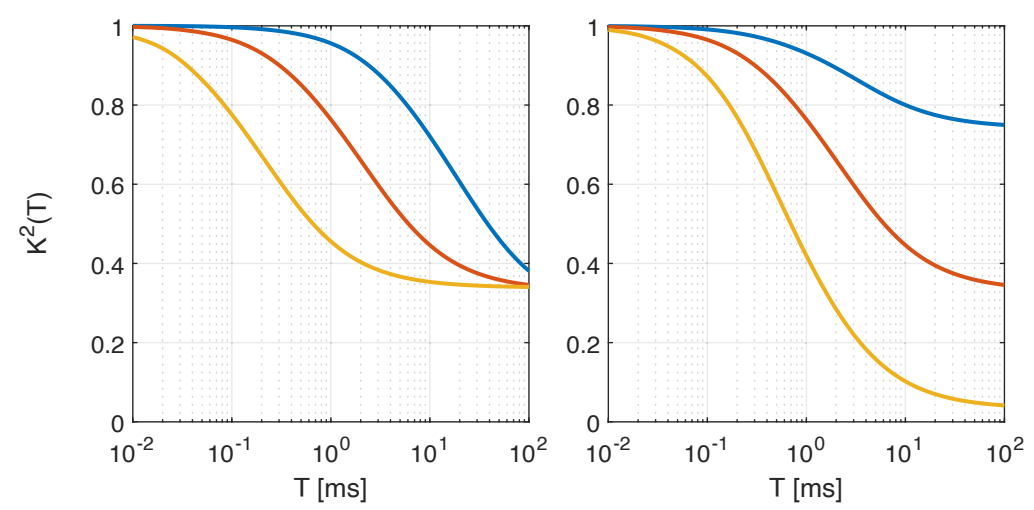

\begin{tabular}{cccccccc}
\hline & \multicolumn{3}{c}{ Changing $\left\langle v_{\mathrm{RBC}}\right\rangle$} & & \multicolumn{3}{c}{ Changing $\rho$} \\
\cline { 2 - 3 } \cline { 6 - 7 } & $\left\langle v_{\mathrm{RBC}}\right\rangle$ & $\rho$ & $(1-\rho)^{2}$ & & $\left\langle v_{\mathrm{RBC}}\right\rangle$ & $\rho$ & $(1-\rho)^{2}$ \\
\hline 0 & 0.26 & 0.42 & 0.34 & & 2.6 & 0.14 & 0.75 \\
$\bigcirc$ & 2.6 & 0.42 & 0.34 & & 2.6 & 0.42 & 0.34 \\
$\bigcirc$ & 26 & 0.42 & 0.34 & & 2.6 & 0.81 & 0.037 \\
\hline
\end{tabular}

Figure 3.6: Example of typical contrast curves for different levels of scatterer speeds (left) and fraction of dynamic scatterers (right). The table summarizes the mean scatterer speed $\left\langle v_{\mathrm{RBC}}\right\rangle[\mathrm{mm} / \mathrm{s}]$, the fraction of Doppler-shifted light $\rho$, and the contrast limit $(1-\rho)^{2}$ at infinite exposure time. Note that the $\mathrm{x}$-axis is in logarithmic scale.

\subsection{Single-exposure laser speckle contrast imaging - LSCI}

As described in Equation 3.4, laser speckle contrast is computed as the standard deviation over average of the intensity of the speckle pattern. In that equation, these quantities were treated as the theoretical properties of the theoretical speckle intensity distribution. Of course, in a real measurement system the speckle pattern intensity is discretized by the pixels of the detector array. The equation for contrast can therefore be rewritten as

$$
\begin{aligned}
\langle I\rangle & =\frac{1}{N} \sum_{i=1}^{N} x_{i} \\
\sigma^{2} & =\frac{1}{N} \sum_{i=1}^{N}\left(x_{i}-\langle I\rangle\right)^{2} \\
K & =\frac{\sigma}{\langle I\rangle},
\end{aligned}
$$


where $x_{i}$ represents the detected intensity value for a single pixel, and the speckle contrast is computed over an $N$-pixel sub-region. This sub-region can in principle be any size, but is usually a square of size $3 \times 3$ to $9 \times 9$ pixels, where $7 \times 7$ appears to be the most common choice [65]. By performing this computation in every sub-region of the speckle image, spatial maps of the contrast are generated. This is essentially a convolution with a non-linear kernel [66].

Since the speckle pattern is sampled in a finite number of pixels, the computed contrast is only an estimate of the true contrast. Increasing the number of pixels in the sub-region improves the estimate, but also decreases the spatial resolution of the contrast images. This trade-off between the size of the contrast kernel and the resolution of the computed image is why the subregion size is usually kept relatively small. At the same time, the number of pixels in the sub-region should be sufficient to accurately represent the true statistics of the speckle pattern. It has been demonstrated that if there are too few speckles in the sub-region, the contrast will be lower than 1 for a static speckle pattern [66]. A the same time, too many speckles per pixel also decreases the maximum contrast due to speckle averaging. In the case of objective speckles, i.e. using an imaging setup, the diameter of the smallest speckle is described by the following equation $[67,68]$ :

$$
d_{s}=1.22 \lambda(1+M) f_{\#}
$$

where $\lambda$ is the laser wavelength, $M$ is the magnification of the imaging setup, and $f_{\#}$ is the f-number of the lens. Increasing the aperture thus results in smaller speckles due to the smaller $f_{\#}$. As mentioned previously, other effects such as polarization of the light will also decrease the maximum value of the contrast. As we already saw in Equation 3.9, all these effects can be incorporated into a calibration constant

$$
\beta=\frac{1}{K_{\max }^{2}},
$$

where $K_{\max }^{2}$ can be experimentally measured on a static scattering object. The multiplication by $\beta$ in Equation 3.9 results in the correct value of $K^{2}(T)=1$ when measuring a static speckle pattern. This is by no means a new discovery, but we also demonstrate the validity of this in Paper III.

Before this calibration, it is common to first remove any contributions to the contrast from measurement noise. Two primary sources of noise are present in speckle images; thermal noise and shot noise [19]. Thermal noise creates false intensity readings even in the absence of any light, hence it is also sometimes called dark noise. The variance contribution from this noise source is constant at a constant temperature, and can be measured from acquired dark images. Shot noise is the fluctuations in detected intensities due to the quantized nature of photons hitting the detector array. This is a Poisson 
process and can therefore be approximated by a normal distribution at large photon counts, with a variance proportional to the intensity. Important for both noise sources is that they are temporally independent, i.e. the noise in one image is independent from other images, regardless of the time delay. This allows us to define a noise model for the computed variance as

$$
\begin{aligned}
\sigma_{\text {measured }}^{2} & =\sigma_{\text {speckle }}^{2}+\sigma_{\text {noise }}^{2} \\
\sigma_{\text {noise }}^{2} & =\langle I\rangle N_{\text {shot }}+N_{\text {dark }},
\end{aligned}
$$

where the noise constants $N_{\text {shot }}$ and $N_{\text {dark }}$ can be measured experimentally

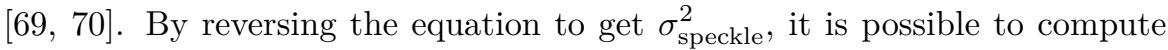
the noise-corrected and calibrated speckle contrast by Equations 3.10c and 3.12 , as

$$
K^{2}(T)=\beta\left(\frac{\sigma_{\text {measured }}^{2}-\sigma_{\text {noise }}^{2}}{\langle I\rangle^{2}}\right) .
$$

\section{LSCI perfusion models}

Ultimately, the speckle contrast is not the parameter of interest for biomedical applications. Rather, it is the tissue perfusion we want to estimate. We therefore require a model to translate from speckle contrast to perfusion. To do this, it is common to model the field autocorrelation function $g^{(1)}(\tau)$ using the parameter $\tau_{c}$, the speckle decorrelation time. This is the time in which the field autocorrelation function, $g^{(1)}(\tau)$, decreases from 1 to $1 / e \approx 0.37$ [15], or in simpler terms, a measure of how long it takes the speckle pattern to no longer be recognizable compared to the reference time ${ }^{1}$. Primarily Gaussian and Lorentzian distributions have been investigated [71], although the Voigt distribution has also been suggested [72]. Assuming a Lorentzian distribution, Equation 3.9 evaluates to [63]

$$
K^{2}(T)=\frac{e^{-2 x}-1+2 x}{2 x^{2}},
$$

and for a Gaussian distribution, Equation 3.9 instead evaluates to

$$
K^{2}(T)=\frac{e^{-2 x^{2}}-1+\sqrt{2 \pi} x \cdot \operatorname{erf}(\sqrt{2} x)}{2 x^{2}},
$$

where $x=T / \tau_{c}$ and "erf" is the Gauss error function [73]. It should be noted that one might find differences in these formulas in different articles, e.g. compare Equation 3.15 and 3.16 with Ref [71]. This is due to a continued use

\footnotetext{
${ }^{1}$ In the cited article (Ref [15]), the authors define $\tau_{c}$ from the autocorrelation function of intensity, i.e. $g^{(2)}(\tau)$. This is most likely a mistake, or a use of non-standard terminology, since $g^{(2)}(\tau)$ by definition is in the range [1,2], and thus cannot "decrease from 1 to $1 / e$ ". Furthermore, the purpose of $\tau_{c}$ is as a model parameter in the expression of the contrast, which is based on $g^{(1)}(\tau)$ (see Equation 3.9).
} 
of the original version of Equation 3.9 where the linear weighting is omitted [63]. It is difficult to say whether or not this omission is intentional, but it is nevertheless clear that there is no strong consensus in the field on which model is the most appropriate.

The exact relation between $\tau_{c}$ and the scatterer speed is not known [74], but the most commonly used approximation defines the "decorrelation velocity" [16]

$$
v_{c}=\frac{\lambda}{2 \pi \tau_{c}},
$$

where $\lambda$ is the laser wavelength. Note that the use of the term "velocity" here is incorrect, as the direction of the flow cannot be estimated, but this is the historical name of the quantity. Essentially, this model claims that $v_{c}$ represents the characteristic speed of the blood, and should therefore be linearly related to the perfusion. The validity of this will be discussed in Chapter 7 . Computing $v_{c}$ requires estimating $\tau_{c}$ from measured contrast, but instead of fitting the models in Equations 3.15 and 3.16 to the data, LSCI perfusion is usually computed by a simple inverse relation $P \propto K^{-n}(T)$, where the choice of either $n=1$ or $n=2$ differs between publications. This form can be motivated empirically by the fact that increasing the flow speed decreases the contrast, meaning the perfusion should be inversely related to the contrast. There is also a theoretical derivation [75] valid for exposure times $T>2 \tau_{c}$, where it has been shown that both the Lorentzian-based and Gaussian-based models of contrast, described in Equations 3.15 and 3.16 (with a minor change in scaling [75]), approximately simplifies so that

$$
P_{\mathrm{SFI}} \equiv \frac{1}{\tau_{c}}=\frac{1}{2 T \cdot K^{2}(T)} .
$$

This specific model is usually called the speckle flow index (SFI), but to keep the notation consistent I will call this $P_{\text {SFI }}$ for perfusion. Note that this model does not approach $P=0$ when $K=1$. Rather, the theoretical lowest value of $P_{\mathrm{SFI}}$ is $1 / 2 T$ for a perfectly calibrated system. Intuitively, since $K=1$ indicates a complete absence of flow, it should correspond to a perfusion of 0 . A simple empirical correction has been proposed, yielding the new perfusion estimate

$$
P_{K}=\frac{1}{K^{n}(T)}-1
$$

It has been shown that this model is more similar to perfusion by LDF for $n=1$, and more similar to true perfusion for $n=2$, although the exact relation is still non-linear and unknown in both cases [54]. Note that both $P_{\text {SFI }}$ and $P_{K}$ assumes that the contrast is already $\beta$-corrected according to Equation 3.14. If this is not the case, an additional calibration constant is required in the numerators.

Regardless of the choice of perfusion model, single-exposure contrast can only provide these relative estimates based on large assumptions, and effects 
such as from static scattering cannot be accounted for. It is also possible for the single exposure contrast to be the same given widely different microcirculatory conditions. This is illustrated in Figure 3.7. For a more powerful model, multiple exposure times are required.
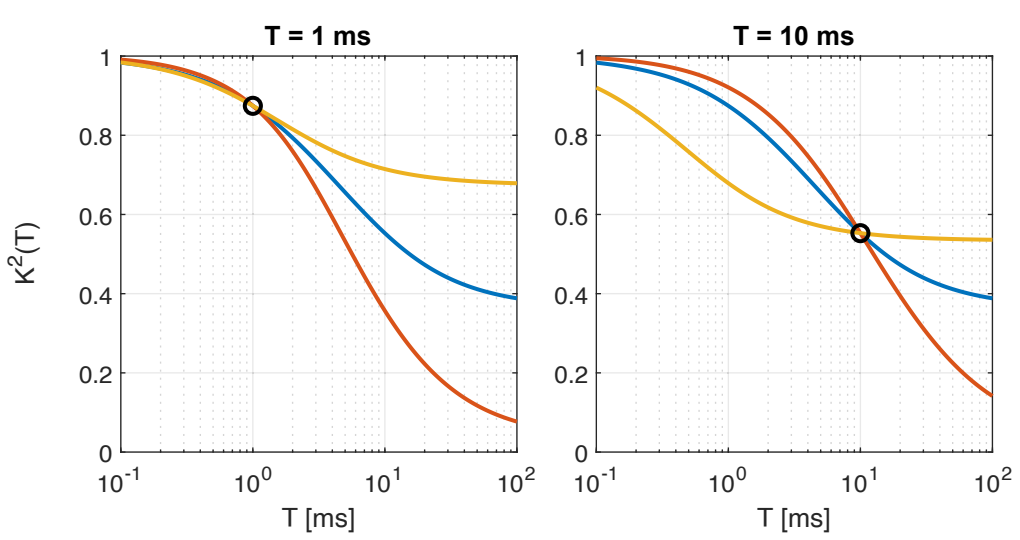

\begin{tabular}{cccccccc}
\hline & \multicolumn{3}{c}{$T=1 \mathrm{~ms}$} & & \multicolumn{3}{c}{$T=10 \mathrm{~ms}$} \\
\cline { 2 - 5 } \cline { 6 - 8 } & $\left\langle v_{\text {RBC }}\right\rangle$ & $c_{\text {blood }}$ & $P_{\text {true }}$ & & $\left\langle v_{\text {RBC }}\right\rangle$ & $c_{\text {blood }}$ & $P_{\text {true }}$ \\
\hline 0 & 0.65 & 0.70 & 0.22 & & 0.65 & 0.70 & 0.22 \\
0 & 0.18 & 1.3 & 0.090 & & 0.21 & 1.4 & 0.033 \\
0 & 2.4 & 0.80 & 0.055 & & 5.3 & 0.58 & 1.1 \\
\hline
\end{tabular}

Figure 3.7: Illustration of why a single exposure time is insufficient to model the microcirculation. The images show that regardless of the exposure time, it is possible to measure the same contrast from widely different microcirculatory situations. The table summarizes some key parameters - mean blood speed, mean blood concentration, and true perfusion. Note that the blue contrast curve is the same in both figures.

\subsection{Multi-exposure laser speckle contrast imaging - MELSCI}

Single-exposure LSCI is limited to relative measurements since the underlying processes that produce the speckles are too complex to be modelled by a contrast measured at a single exposure time. For this reason, Parthasarathy et al. [19] proposed multi-exposure laser speckle contrast imaging (MELSCI), which measures the contrast at multiple exposure times to enable more sophisticated models based on the increased information. MELSCI is in essence very similar to single-exposure LSCI, in that it quantifies the motion blur in 
the speckle images, which then is connected to the blood flow via a perfusion model. The main difference is, of course, in the data collection.

There have been two main methods proposed for collecting multi-exposure images; by modulating the light source, or by synthetic exposure times, both of which will be explained briefly below. Interestingly, the naive approach where the exposure time is varied by changing the camera exposure directly, has not been used in publications. The main reason for this is to prevent the noise characteristics from changing with the exposure time. Instead, the first proposed MELSCI system used a fixed camera exposure $T_{\max }$ and changed the effective exposure time by modulating the laser intensity and duration [19], as described in Ref [76]. This method ensures that accumulated dark noise $N_{\text {dark }}$ is the same for all exposure times since the camera exposure is equal, and that the shot noise $N_{\text {shot }}$ is the same since the total intensity is equal. This enables using the same noise correction for all exposure times, using Equation 3.13. A schematic description of this acquisition scheme is depicted in Figure 3.8a.

A major disadvantage of the modulated method is that each exposure time is captured at separate absolute times. Since each image is exposed for the maximum exposure time $T_{\max }$, if $N$ exposure times are required, the minimum capture time is $N T_{\max }$. This is most likely a relatively long time compared to the dynamics of the speckle pattern, which introduces statistical uncertainty in the models. The method also requires a strict synchronization between the camera and laser to correctly capture each exposure window. This makes the experimental setups more complicated than necessary.

To address both of these issues a new acquisition scheme was proposed by Dragojević et al. [21], in which only the shortest exposure time was captured, but fast enough that new exposure times could be created by digitally adding successive images in the post-processing. This method allows a constant laser illumination, removing any synchronization requirements between laser and camera. Furthermore, the total acquisition time is simply $T_{\max }$, at least $N$ times faster than the modulated approach, allowing for much greater time-resolution in the final perfusion images. Even more significant is that all exposure times are computed from the same data, essentially meaning they are acquired at the same time instead of sequentially. All contrast images within one multi-exposure set is therefore from the same realization of the speckle pattern, eliminating the statistical uncertainty present in the modulated acquisition. This acquisition scheme is illustrated in $3.8 \mathrm{~b}$, and was the core algorithm for the MELSCI implementation in this thesis. In Paper I, this was implemented in programmable logic hardware to enable real-time analysis of MELSCI data. 


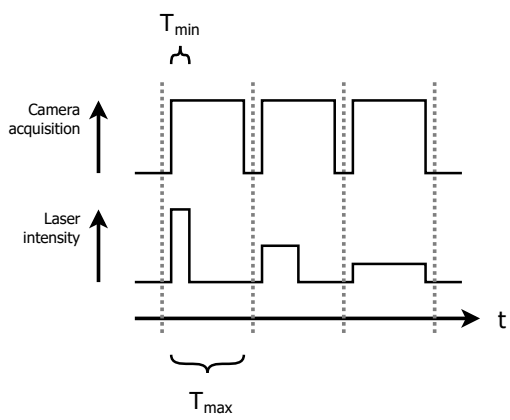

(a) Modulated laser source

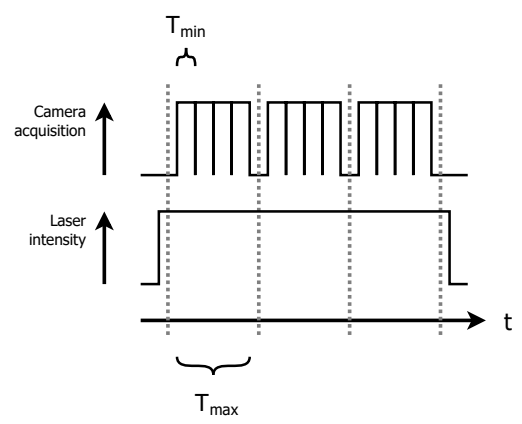

(b) Synthetic exposure times

Figure 3.8: Schematic overview of the two main methods of acquiring multi-exposure data in MELSCI. In (a), the camera exposure is fixed at the longest exposure time $T_{\max }$, while the effective exposure is changed by modulating the intensity and duration of the laser illumination. In (b), the camera exposure is instead fixed at the shortest exposure time $T_{\min }$, and the laser illumination is constant. By digitally adding the data from two or more adjacent images, synthetic exposures at any multiple of $T_{\min }$, up to $T_{\max }$, can be created. Note that in (a), only a single multi-exposure set is captured, while three independent multiexposure sets are captured in the same time in (b).

\section{Expanded multi-exposure noise model}

Multi-exposure contrasts, just like single-exposure contrasts, must be corrected for noise variance before applying a perfusion model. If the modulated laser acquisition is used (Figure 3.8a), the dark noise and shot noise can be designed to be equal for all exposure times, enabling the direct use of Equations 3.13 and 3.14 in the same way as for single-exposure measurements. If the second approach is used (Figure 3.8b), which creates synthetic exposure times by adding acquired images, the noise parameters $N_{\text {dark }}$ and $N_{\text {shot }}$ should be measured for each exposure time and applied separately, since the accumulated dark counts and shot noise variance will differ. Alternatively, a joint model can be applied in which the same noise parameters are used for all exposure times after $T$ has been properly added to the model. We presented this model in Paper IV, but left out some finer details. I will therefore use this opportunity to present the model again with some measured data for validation.

Since the dark counts in two consecutive frames are independent random variables, their variances are additive. This means that the total variance contribution from this noise source scales linearly with the amount of added images $n=T / T_{\min }$. The same is true for shot noise, where the total noise 
contribution scales with image intensity, which by nature of the synthetic exposure times and constant illumination is $n\langle I\rangle$, i.e. the intensity of the acquired images multiplied by the amount of added images.

If there is a constant source of variance $E_{\text {const }}$ in the images, this is included in the dark noise parameter for the single-exposure model. However, for the joint multi-exposure model this must be considered separately from the dark noise. This is because the variance contribution from a constant source scales with the square of number of added images $n$. This variance can for example be due to an uneven illumination by the laser or a miscalibrated sensor, such that it will be the same regardless of the object that is imaged. Since this is not a fundamentally unavoidable noise source in the same way that dark noise and shot noise is, an effort should be made to minimize it if possible. However, due to the quadratic scaling with $T$, it should probably also be measured and corrected for, otherwise it might overpower the speckle variance at long exposure times and low intensities. Combining these three noise sources, we can propose the joint model

$$
\sigma_{\text {noise }}^{2}(T)=\frac{T}{T_{\min }}\langle I\rangle N_{\text {shot }}+\frac{T}{T_{\min }} N_{\text {dark }}+\left(\frac{T}{T_{\min }}\right)^{2} E_{\text {const }} .
$$

Note that in Paper IV we use $T_{\min }=1 \mathrm{~ms}$, and therefore the published model does not include this factor. Regardless, for each exposure time, this model will be a $1^{\text {st }}$-degree polynomial of $\langle I\rangle$, with slope proportional to $N_{\text {shot }}$ and intercept at some value $\sigma_{\text {intercept }}^{2}(T)$. By setting $\langle I\rangle=0$ in the above equation we see that the intercepts at different exposures should follow a $2^{\text {nd }}$-degree polynomial of the variable $T$, should pass through the origin, and where the linear component is from the dark noise and the quadratic component is from the constant variance source. The validity of this model can easily be investigated by fitting a straight line to noise variance at different synthetic exposure times measured at a range of intensities, and then fitting a model $a T+b T^{2}$ to the intercepts of the individual lines. This is shown in Figure 3.9 , where we can observe that this model fits the data well. After validating the intercept model, the full joint model can be fit to the noise variance at different exposure times and intensities, as shown in Figure 3.10. Once again we observe a very good fit between the model and measured data, with only a slight deviation for $T=1 \mathrm{~ms}$ at low intensities. With this joint model, three parameters are sufficient to explain the noise characteristics for all exposure times and intensities. The final step is to compute the corrected contrast according to Equation 3.14.

The noise parameters that were computed from this model and used in Paper IV and onward are $N_{\text {shot }}=0.348, N_{\text {dark }}=81.5$, and $E_{\text {const }}=4.31$. 

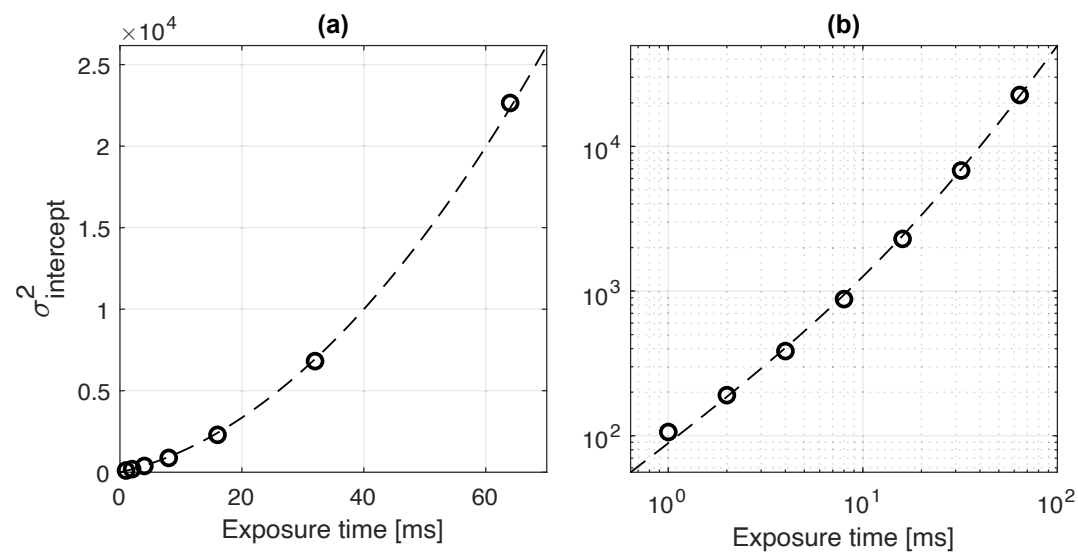

Figure 3.9: (a) Fit on the form $\sigma_{\text {intercept }}^{2}=a T+b T^{2}$ to intercepts of the seven 1st-degree polynomials individually fit to noise data at each exposure time. The relative error was 5.7\%. (b) Loglog-plot of the same data for increased visibility.
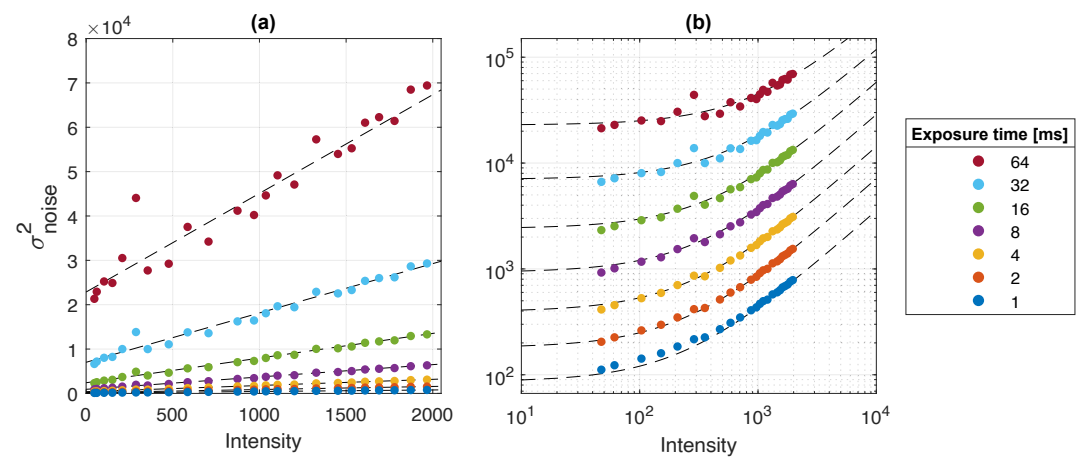

Figure 3.10: (a) Joint noise model fit to measured noise variance for a wide range of intensities. The noise at each exposure time $T$ is described by a line with slope $T N_{\text {shot }}$ and intercept $T N_{\text {dark }}+T^{2} E_{\text {const }}$. The relative model error was $4.7 \%$. (b) Loglog-plot of the same data for increased visibility. 


\section{MELSCI perfusion models}

Notice that the contrast models in Equations 3.15 and 3.16, resulting from the assumed $g^{(1)}(\tau)$ distributions, both approach 0 as $T$ goes to infinity. This corresponds to that all detected light has been Doppler-shifted by a moving scatterer at least once, i.e. the Doppler fraction $\rho=1$. This is a necessary assumption in single-exposure LSCI since the Doppler fraction can only be measured by using a long exposure time (see Figure 3.6), which then severely diminishes the sensitivity to the flow speed. As several authors have discussed, this assumption is only valid at very high blood concentrations, which does not conform with most biological tissues $[19,77]$. It is possible to extend the contrast models to include the effects of static scattering, such that $K^{2}(T)$ becomes a function of $\tau_{c}$ and $\rho$. This is done by assuming the detected light is a mix of static and dynamically scattered light $I=I_{\mathrm{s}}+I_{\mathrm{d}}$, and then computing the autocorrelation of $I$, which yeilds $g^{(2)}(\tau)$. From here, Equation 3.7 and 3.9 can be used to give a model of the contrast. By again assuming a Lorentzian distribution for $g^{(1)}(\tau)$, we get

$$
K^{2}(T)=\beta\left(\rho^{2} \frac{e^{-2 x}-1+2 x}{2 x^{2}}+4 \rho(1-\rho) \frac{e^{-x}-1+x}{x^{2}}+(1-\rho)^{2}\right)+\nu_{n},
$$

where $x=T / \tau_{c}$ and $\nu_{n}$ is a residual term that incorporates any remaining contrast that is unaccounted for by the model. The full details of this derivation is beyond the scope of this thesis, but can be found in Ref [39], with some excellent complementary derivations in Ref [63]. The optimal parameters can be estimated by fitting the model to measured data, after which the perfusion can be computed from either of the following equations:

$$
\begin{aligned}
& P_{\mathrm{ME}}=\frac{1}{\tau_{c}} \\
& P_{\mathrm{ME}}=\frac{\rho}{\tau_{c}},
\end{aligned}
$$

where ME stands for multi-exposure. The first model is essentially the speckle flow index corrected for static scattering, and the second is an attempt to more accurately estimate true perfusion, which also depends on the blood concentration [Paper II]. The first is usually seen in publications [21, 22, 39], together with the connection to the decorrelation velocity $v_{c}$. However, when applying the model in Paper II and Paper IV, we used the second version to include the effects of the blood concentration. 


\section{4}

\section{Computational model of skin}

The models for contrast and perfusion in the previous chapter are analytical models that directly estimate the respective quantities in a single equation. The notable exception is the MELSCI perfusion, which requires fitting the model to measured contrast data using an iterative optimizer, for example the Levenberg-Marquardt algorithm [78, 79]. In both cases however, this approach might be overly restrictive given the complicated nature of the microcirculation and surrounding tissue. Multiple assumptions made in those models have been questioned [72, 80], or demonstrated to be inaccurate [81]. Furthermore, the model parameters, for example $\tau_{c}$, are not the physiologically relevant quantities of interest, such as blood concentration and RBC speed distribution, which makes the interpretation of the optimal parameters more difficult. Figure 4.1 illustrates this, by demonstrating how the contrast depends directly on the properties of a simulated multi-layered skin model. We can observe that $\rho$, for example, is a complicated parameter described by some combination of the more fundamental properties of skin, such as scattering coefficient $\mu_{s}$, blood concentration $c_{\text {blood }}$, and vessel diameter $d_{\text {vessles }}$. A similar argument can be made for $\tau_{c}$, which is most affected by the blood concentration $c_{\text {blood }}$ and the speed of red blood cells $v_{\text {RBC }}$, but not only these. Other properties of skin, such as the absorption $\mu_{a}^{\mathrm{epi}}$ and blood oxygen saturation $S_{\text {oxy }}$, has a minimal effect on the contrast.

Ideally, a model for contrast and perfusion should be based on these properties of the skin, but given the complicated nature, such a model can likely not be stated in an analytical expression. The alternative is to use a computational model, i.e. a model where the contrast or perfusion is computed from the tissue properties, for example using Monte Carlo light-transport simulations and a framework of mathematical transformations [82], instead of a single analytical expression. This allows more complicated effects to be taken into account, such as multiple scattering and discrete vessels. Perfusion can be estimated from measured contrast by iteratively fitting the model to the measurements. Alternatively, as has been done in this thesis, machine learning can be used to obtain a direct transfer function from measured contrast to perfusion. This chapter explains the skin model and the mathematical framework. 

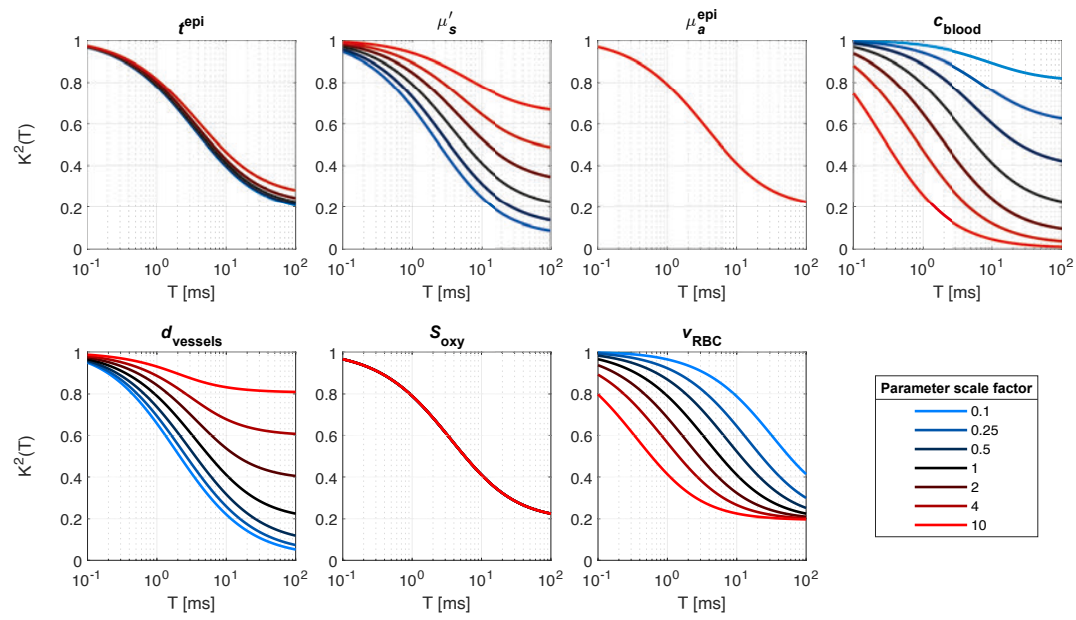

Figure 4.1: Influence of several properties of skin on the contrast $K^{2}(T)$ as a function of exposure time. A base model of skin (explained in this chapter) was modified by scaling one parameter at a time, indicated by the plot titles, by the factors in the legend, while the other parameters were kept constant. The black line is identical in all plots, corresponding to the base model. The blood concentration $c_{\text {blood }}$, vessel diameter $d_{\text {vessels }}$, and oxygen saturation $S_{\text {oxy }}$ are aggregated values from the multilayered skin model described in this chapter. A few cases, for example $t^{\text {epi }}$ scaled by 10 , was outside the defined range of the model and was thus excluded.

\subsection{Multi-layered skin model}

The skin model consists of three layers; a variable-thickness bloodless epidermis layer, a $0.2 \mathrm{~mm}$ upper dermis layer, and an infinite lower dermis layer. In total, the model is described by 26 parameters. However, some of these are only relevant for spectroscopic measurements. Due to the single-wavelength nature of MELSCI, only 20 parameters are of interest here. As such, there are some minor differences in the description of the model compared to published papers, for example Ref [83]. The parameters with relevance to MELSCI are:

- Epidermis thickness, $t^{\text {epi }}$

- Reduced scattering coefficient, $\mu_{s}^{\prime}$

- Epidermis absorption, $\mu_{a}^{\text {epi }}$

- Upper dermis blood concentration, $c_{\mathrm{blood}}^{\mathrm{U}}$

- Lower dermis blood concentration, $c_{\text {blood }}^{\mathrm{L}}$ 
- Upper dermis vessel diameter, $d_{\mathrm{vessels}}^{\mathrm{U}}$

- Lower dermis vessel diameter, $d_{\text {vessels }}^{\mathrm{L}}$

- Upper dermis blood oxygen saturation, $S_{\text {oxy }}^{\mathrm{U}}$

- Lower dermis blood oxygen saturation, $S_{\text {oxy }}^{\mathrm{L}}$

- 11 parameters $k_{v}$ and $w_{i}$ for $i \in[1,10]$, for the speed distribution of RBCs described below

An important note is that the parameters for the upper and lower dermis layers are coupled by a mean value and a difference, in order to constrain the model to more realistic configurations. As such, not all combinations of, for example, $c_{\text {blood }}^{\mathrm{U}}$ and $c_{\text {blood }}^{\mathrm{L}}$ are equally likely in the randomized model below. For a more detailed description, see Ref [84]. The absorption $\mu_{a}^{\mathrm{U}}$ and $\mu_{a}^{\mathrm{L}}$ in the respective dermis layer is computed by the corresponding $c_{\text {blood }}, d_{\text {vessels }}$, and $S_{\text {oxy }}$. The distributions of parameters in 100000 randomized models are presented in Figure 4.2. For the dermis-parameters, the mean is presented.

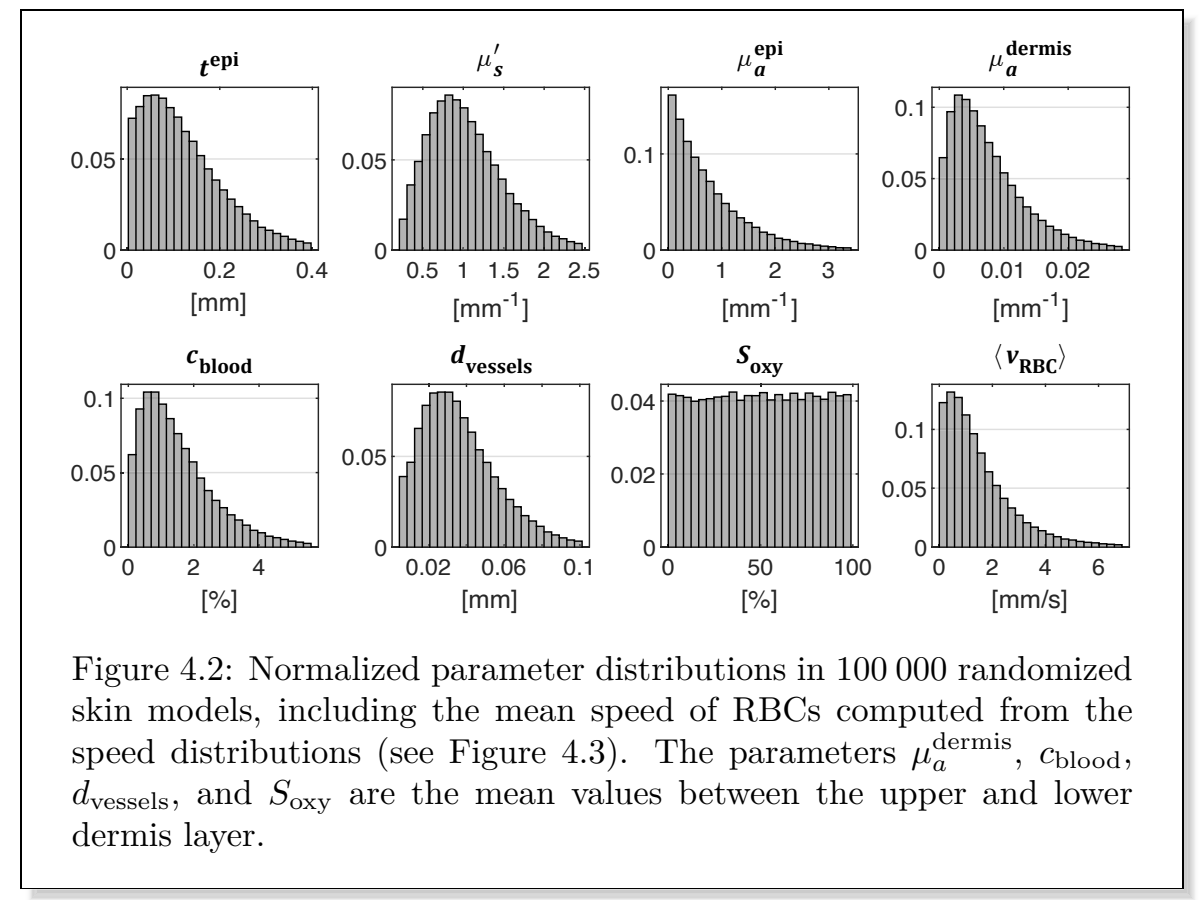

The speed distribution of RBCs is a weighted sum of 10 uniform distributions $c_{\mathrm{RBC}, i}(v)$, each from 0 to $2 v_{i}$, where the average speeds $v_{i}$ are distributed 
linearly in the logarithmic plane according to

$$
\begin{aligned}
v_{i} & =k_{v} \cdot v_{\text {min }} \cdot 10^{(i-1) v_{\text {step }}} \\
v_{\text {min }} & =0.2 \mathrm{~mm} / \mathrm{s} \\
v_{\text {max }} & =75 \mathrm{~mm} / \mathrm{s} \\
v_{\text {step }} & =\frac{1}{9} \log _{10}\left(\frac{v_{\text {max }}}{v_{\text {min }}}\right) \approx 0.286,
\end{aligned}
$$

and $k_{v}$ is a scale factor that scales all 10 uniform distributions equally [54]. To further clarify, if we assume $k_{v}=1$, the first uniform distribution has a span $[0,0.4] \mathrm{mm} / \mathrm{s}$, and the last has a span $[0,150] \mathrm{mm} / \mathrm{s}$. The combined speed distribution is given by a weighted sum

$$
c_{\mathrm{RBC}}(v)=\sum_{i=1}^{10} w_{i} \cdot c_{\mathrm{RBC}, i}(v),
$$

where the weights $w_{i}$ are randomized to simulate different tissues. The weights sum to 1 and - by nature of the uniform distributions - so does the combined speed distribution. A single uniform distribution represents a vessel with a parabolic flow profile. The combined speed distribution thus represents a collection of vessels with parabolic flow profiles with 10 distinct average speeds. Naturally, this is a simplification, but although the amount of uniform distributions can be changed, 10 allows for very flexible distributions while being computationally efficient. A few example speed distributions are presented in Figure 4.3.

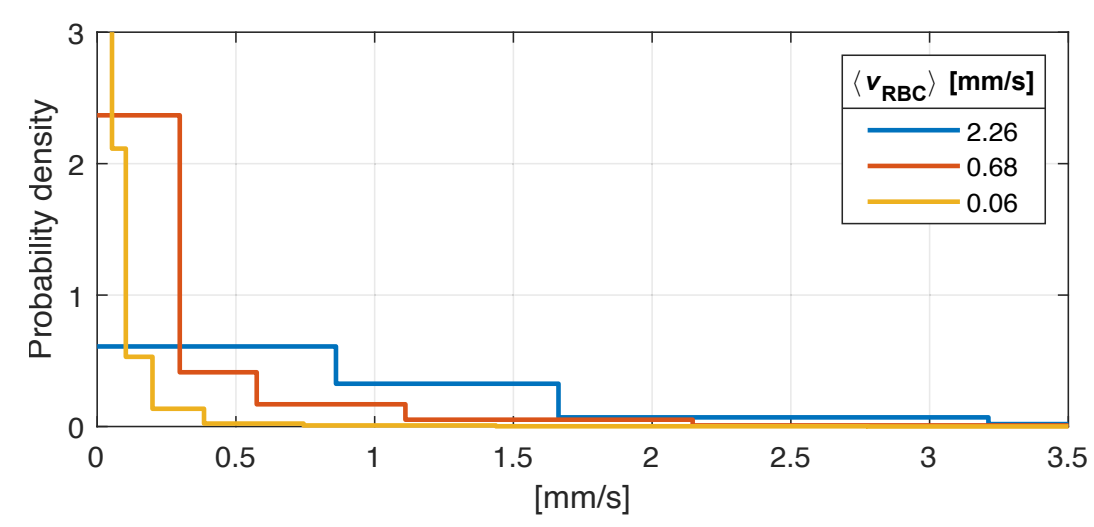

Figure 4.3: Examples of RBC speed distributions $c_{\mathrm{RBC}}(v)$. The average speed is summarized in the figure legend. Note that the distributions extend to much higher top-speeds than the figure limit. 


\subsection{Overview of the computational model}

Figure 4.4 presents an overview of the computational model, from presimulated path-length distributions, speed distribution, and skin properties, to noise-modified contrast and perfusion measures that are used to train the artificial neural networks (ANNs). The flow chart includes references to the relevant equations, mostly in this chapter, but also some that were covered in the previous chapter.

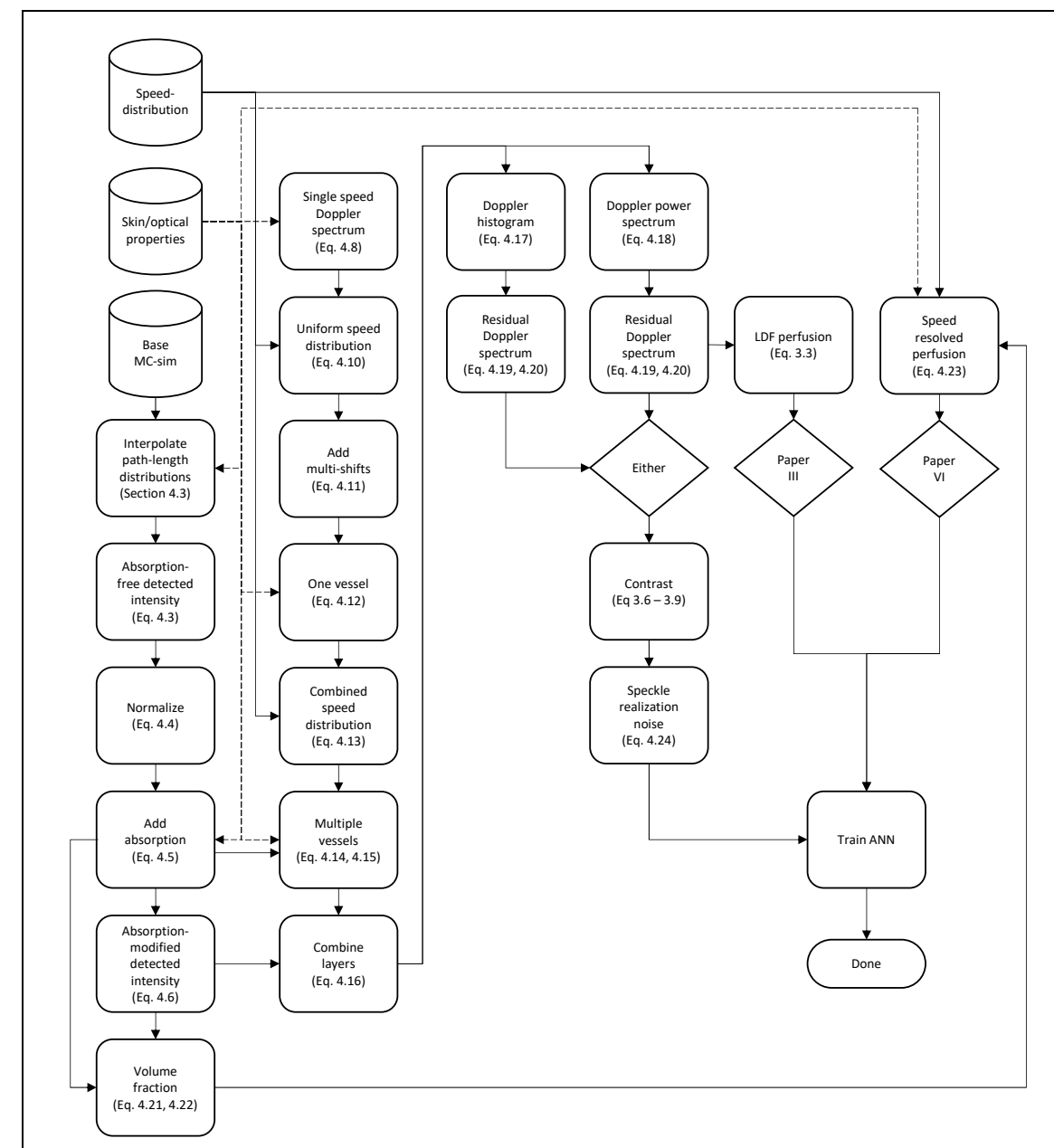

Figure 4.4: Overview of the computational model. 


\subsection{Path-length distributions and measured intensity}

For a given epidermis thickness and scattering coefficient, the model requires the distribution of photon path-lengths, for photons that have propagated through different combinations of the three skin layers. Specifically, we require the path-length distribution $p_{\mathrm{pl}, m, n}$, describing the intensity (i.e. number of photons) of path-lengths $l$ in layer $m$, for all photons that have reached layer $n$ at their deepest. The layers are numbered from 0 to 2 in order epidermis, upper dermis, and lower dermis. This results in six distributions, since $m \leq n$. For example, $p_{\mathrm{pl}, 0,1}$ is the distribution of path-lengths in the epidermis layer $(m=0)$, for photons that have at some point been in the upper dermis layer $(n=1)$, but not in the lower dermis layer $(n \neq 2)$. A set of Monte Carlo simulations were run for 14 values of $t^{\text {epi }}$ and 14 values of $\mu_{s}^{\prime}$, and the pathlength distributions of detected photons were stored for each combination of $m$ and $n$ [83]. The path-length distributions for other values of $t^{\text {epi }}$ and $\mu_{s}^{\prime}$ were then interpolated from the stored distributions. The normalized pathlength distributions (Equation 4.4) for $t^{\mathrm{epi}}=0.1 \mathrm{~mm}$ and $\mu_{s}^{\prime}=1.6 \mathrm{~mm}^{-1}$ is presented in Figure 4.5. This methodology has been validated in Ref [85].
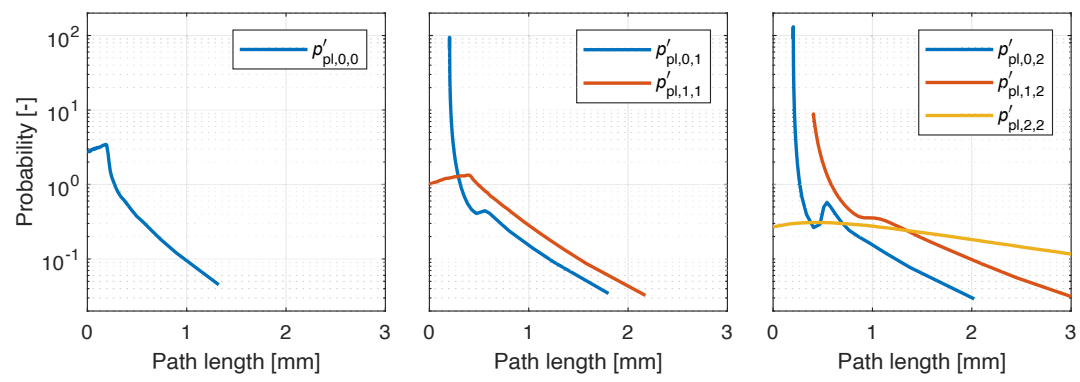

Figure 4.5: Example of normalized path-length distributions for $t^{\text {epi }}=0.1 \mathrm{~mm}$ and $\mu_{s}^{\prime}=1.6 \mathrm{~mm}^{-1}$, obtained by interpolating from a set of base path-length distributions computed by Monte Carlo simulations of photon transport in the skin models, and normalizing according to Equation 4.4. Distribution $p_{\mathrm{pl}, m, n}^{\prime}$ reflects the path-lengths in skin layer $m$, for photons that have reached layer $n$, but not deeper. For example, observe that $p_{\mathrm{pl}, 0,1}^{\prime}$ starts at $l=0.2 \mathrm{~mm}$, i.e. $2 t^{\mathrm{epi}}$, representing the minimum path-length in epidermis for photons that have at least been in the upper dermis layer. Similarly, $p_{\mathrm{pl}, 1,2}^{\prime}$ begins at $l=0.4 \mathrm{~mm}$, i.e. the minimum path-length in the upper dermis layer (which is $0.2 \mathrm{~mm}$ thick), for photons that have at some point been in the lower dermis layer. 
The absorption-free detected intensity of photons where the lowest layer reached was $n$, is given by

$$
I_{0, n}=\sum_{l} p_{\mathrm{pl}, m, n}(l),
$$

where any $m \leq n$ gives the same intensity $I_{0, n}$, since they contain the same photons. The distributions are then normalized by

$$
p_{\mathrm{pl}, m, n}^{\prime}(l)=p_{\mathrm{pl}, m, n}(l) / I_{0, n},
$$

and absorption is added by Beer-Lambert's law for each path-length $l$ :

$$
p_{\mathrm{pl}, m, n}^{\prime \prime}(l)=p_{\mathrm{pl}, m, n}^{\prime}(l) e^{-l \mu_{a}^{(m)}},
$$

where $\mu_{a}^{(m)}$ is the absorption in skin layer $m$. Finally, the absorption-modified detected intensity for all photons where the lowest layer reached was $n$, is given by

$$
I_{n}=I_{0, n} \prod_{m=1}^{n} \sum_{l} p_{\mathrm{pl}, m, n}^{\prime \prime}(l),
$$

and the total detected intensity is

$$
I=\sum_{n} I_{n}
$$

\subsection{Computing the Doppler spectrum}

The Doppler power spectrum $P(f)$ is the base for computing both the contrast and conventional LDF perfusion. Alternatively, the contrast can be computed from the Doppler histogram $H(f)$. To arrive at $H(f)$ and $P(f)$, we begin with the Doppler histogram for a single RBC speed $v$, denoted $p_{o, v}(f)$. The frequency shift of a scattering event is given by

$$
\begin{aligned}
f=\mathbf{v} \cdot \mathbf{q} & =\frac{2 n v}{\lambda} \sin \left(\frac{\theta}{2}\right) \cos (\varphi) \\
& =\frac{2 n v}{\lambda} \sqrt{\frac{1-\cos (\theta)}{2}} \cos (\varphi) \\
& =\left[\begin{array}{l}
\mu=\cos (\theta) \\
\xi=\cos (\varphi)
\end{array}\right]=\frac{2 n v}{\lambda} \sqrt{\frac{1-\mu}{2}} \xi
\end{aligned}
$$

where the definition of vectors and angles have been presented in Chapter 3, Figure 3.2. The distribution of $f$ can therefore be computed from the distributions of $\mu$ and $\xi$. Due to a combination of the complex structure of the microvascular network and the diffuse scattering regime in which the Doppler shifts occur, it is reasonable to assume isotropic angles $\varphi$ between $\mathbf{v}$ and $\mathbf{q}$. Furthermore, since the frequency distribution is symmetric around 0 , we can 
consider only positive Doppler shifts. The distribution of $\xi$ is then uniform $\xi \in[0,1]$. The distribution of $\mu$, i.e. the cosine of the angle change of the light path, can be calculated from the scattering phase function of blood. Here, a Gegenbauer kernel with $g_{\mathrm{Gk}}=0.948$ and $\alpha_{\mathrm{Gk}}=1$ is used, which is relevant at a wavelength of $780 \mathrm{~nm}$ [86]. For the full derivation of the expression for $p_{o, v}(f)$, see the Appendix in Ref [83].

As stated previously, $p_{o, v}(f)$ is the single-shifted Doppler histogram for a single speed $v$. We now consider a single vessel with a parabolic flow profile, i.e. a uniform speed distribution $c_{\mathrm{RBC}}(v) \in[0,2 v]$ where $v$ is the average speed in the vessel. This is calculated by averaging several $p_{o, v_{j}}(f)$ calculated from a sufficiently large set of samples from the uniform distribution. We therefore select $\zeta_{v}$ equally spaced speeds

$$
v_{j} \in\left[\frac{v}{\zeta_{v}}, \frac{3 v}{\zeta_{v}}, \frac{5 v}{\zeta_{v}}, \ldots, \frac{\left(2 \zeta_{v}-1\right) v}{\zeta_{v}}\right],
$$

where $\zeta_{v}$ is a constant that can be changed based on the implementation, as long as it is sufficiently large. For the rest of this chapter, implementation constants of this nature are denoted with $\zeta$, for consistency. In the current implementation, we use $\zeta_{v}=10$. From this, we can compute the single-shifted Doppler histogram for a uniform speed-distribution with average speed $v$ [83]:

$$
y_{1, v}(f)=\frac{1}{\zeta_{v}} \sum_{j=1}^{\zeta_{v}} p_{o, v_{j}}(f) .
$$

The $s$-times multiple-shifted Doppler histogram $y_{s, v}(f)$ is obtained by crosscorrelating $y_{1, v}(f)$ with itself $s$ times. This can be performed more efficiently via the Fourier transform, as

$$
Y_{v}(\gamma) \equiv \mathcal{F}\left(y_{1, v}(f)\right) \Longrightarrow \mathcal{F}\left(y_{s, v}(f)\right)=Y_{v}^{s}(\gamma) .
$$

Given a vessel with diameter $d$, the number of scattering events in that vessel can be assumed to follow a Poisson distribution $p_{\text {Po }}$ with expectation value $\hat{n}_{\text {shifts }}=d \mu_{s}^{\text {blood }}$ [83], where $\mu_{s}^{\text {blood }}=222 \mathrm{~mm}^{-1}$ is the scattering coefficient for blood at the laser wavelength $780 \mathrm{~nm}[86,87]$. Combined with the previous equation, we get the Fourier transformed Doppler histogram from one vessel:

$$
Y_{\text {vessel }, v}(\gamma)=\sum_{s=0}^{\zeta_{s}} Y_{v}^{s}(\gamma) \cdot p_{\text {Po }}\left(s, \hat{n}_{\text {shifts }}\right),
$$

where we assume that at most $\zeta_{s}$ shifts can occur in a vessel.

Now recall that the complete speed distribution is a weighted sum of $\zeta_{u}=10$ uniform distributions. The combined Fourier-transformed multipleshifted Doppler histogram for the full speed-distribution can therefore be computed as

$$
Y_{\text {vessel }}(\gamma)=\sum_{i=1}^{\zeta_{u}} w_{i} Y_{\text {vessel }, v_{i}}(\gamma),
$$


for mean speeds $v_{i}$ and weights $w_{i}$. Note that this still only considers a single vessel, although the full speed-distribution is included by the probabilities $w_{i}$. To include the effect of multiple vessels, we use the path-length distributions described in the previous section. For a given path-length $l_{j}$, vessel diameter $d$, and blood volume fraction $f_{\text {blood, } m}$ in layer $m$, the number of vessels $\eta$ that are intersected by the path can be considered to follow a Poisson distribution $p_{\mathrm{Po}}\left(\eta, \mu_{\mathrm{vessels}, m}\right)$, where $\mu_{\mathrm{vessels}, m}=l_{j} f_{\mathrm{blood}, m} / d[83]$. Combining this with the absorption-modified path-length distribution $p_{\mathrm{pl}, m, n}^{\prime \prime}(l)$, we get the distribution of intersected vessels

$$
p_{\text {vessels }, m, n}(\eta)=\sum_{j}\left(\frac{p_{\mathrm{pl}, m, n}^{\prime \prime}\left(l_{j}\right)}{\sum_{i} p_{\mathrm{pl}, m, n}^{\prime \prime}\left(l_{i}\right)} \cdot p_{\mathrm{Po}}\left(\eta, \mu_{\mathrm{vessels}, m}\right)\right),
$$

where we normalize the path-length distribution to remove the dependency on detected intensity. This can now be combined with Equation 4.13 to get the Fourier-transformed Doppler histogram when considering multiple vessels:

$$
Y_{\mathrm{ms}, m, n}(\gamma)= \begin{cases}1 & \text { for } m=0 \\ \sum_{\eta=0}^{\zeta_{\eta}} p_{\mathrm{vessels}, m, n}(\eta) Y_{\mathrm{vessel}}^{\eta}(\gamma) & \text { for } m>0\end{cases}
$$

where $m=0$ indicates the light from the epidermis layer. Since this layer is bloodless, the Doppler histogram of this light is a Dirac delta at $0 \mathrm{~Hz}$, which corresponds to a constant 1 for all $\gamma$ in the Fourier domain. Similar to before, we assume that a maximum of $\zeta_{\eta}$ vessels are intersected. This method of including multiple vessels have been shown to compensate well for the vessel packaging effect, i.e. the effect from considering discrete vessels instead of a homogeneous distribution of blood everywhere in the dermis layers [88].

Next, the contributions from each layer is combined, weighted by the relative detected intensity $I_{n}^{\prime}=I_{n} / \sum_{j} I_{j}$ from the respective layers (see Equation 4.6). This is done by cross-correlating the optical Doppler spectra, which in the Fourier domain is a product:

$$
Y_{\mathrm{ml}}(\gamma)=\sum_{n=0}^{2}\left(I_{n}^{\prime} \prod_{m=1}^{n} Y_{\mathrm{ms}, m, n}(\gamma)\right)
$$

The final step is to compute the Doppler histogram

$$
H(f)=\mathcal{F}^{-1}\left(Y_{\mathrm{ml}}(\gamma)\right)
$$

or the Doppler power spectrum

$$
P(f)=\mathcal{F}^{-1}\left(Y_{\mathrm{ml}}^{2}(\gamma)\right)
$$

both of which can then be used to compute the multi-exposure contrast $K^{2}(T)$ as in Equations 3.6 to 3.9. 


\subsection{Residual Doppler spectrum}

Empirical results from currently ongoing, not yet published, research in our group has indicated that when fitting this model to in-vivo measurements of the Doppler power spectrum $P(f)$ at low flow conditions, for example during brachial occlusion, there is a persistent residual between the measured and modeled spectra. This has been verified using LDF data collected during occlusion-release experiments as part of the Swedish CArdioPulmonary bioImage Study (SCAPIS) [89], specifically SCAPIS Micro (e.g. Ref [90]) at Linköping University Hospital (Linköping, Sweden). Although we currently have no physiological explanation of this phenomenon, it is clear that is must be included in the model to ensure a good fit between measured and modeled $P(f)$ for low-perfusion data. Although MELSCI does not directly measure the Doppler power spectrum, the same effect is observed in these measurement as well. Including the effect in $H(f)$ before computing the contrast significantly improves the quality of perfusion estimates in terms of noise in low-perfusion settings. Importantly, this effect is not observed in phantom measurements, where flow is measured in plastic tubes perfused with whole blood. This rules out any effects from the measurement device, such as a potentially faulty laser source, and strongly indicates that the effect is physiological in nature, but not originating from the blood itself. However, further research is required to understand this effect.

Regardless of the origin, the effect, which we for now will call the residual Doppler spectrum $R_{D}(f)$, can be modeled as a linearly decreasing function in the logarithmic plane. Empirically, the following model resembles the residual well:

$$
\begin{aligned}
L(f) & =\xi_{\text {offset }}+\xi_{\text {slope }} \cdot f \\
R_{D}(f) & = \begin{cases}0 & \text { for } f=0 \\
\alpha \cdot 10^{L(f)} & \text { for } f>0\end{cases}
\end{aligned}
$$

where $\xi_{\text {offset }} \in \mathcal{N}(-5,0.125)$ and $\xi_{\text {slope }} \in \mathcal{N}\left(-5.2 \cdot 10^{-5}, 6 \cdot 10^{-6}\right)$ are samples from the respective normal distributions $\mathcal{N}(\mu, \sigma)$, estimated from measured data $^{1}$. Using the random variables $\xi$ in the model allows for variations in the magnitude and shape of the noise spectra, which is also observed in measurements due to variation between individuals. $L(f)$ is thus a linearly decreasing function of $f$, with randomized slope and offset. The scale factor $\alpha$ is equal to 1 when applying $R_{D}(f)$ to $H(f)$, and 2 when applying $R_{D}(f)$ to $P(f)$. We also specify $R_{D}(0)=0$, to retain the fraction of non-Doppler shifted photons

\footnotetext{
${ }^{1}$ These values are valid when using NFFT $=8192$ points in the fast Fourier transforms. If NFFT is changed, the magnitude of the residual spectrum should be changed inversely proportional to this, since the total probability over all frequencies is 1 , and thus the probability of a single frequency bin is inversely proportional to NFFT.
} 
$1-\rho$. After applying the noise model

$$
\begin{gathered}
\tilde{H}(f)=H(f)+R_{D}(f) \\
\tilde{P}(f)=P(f)+R_{D}(f)
\end{gathered}
$$

the Doppler-shifted portion of $\tilde{H}(f)$ and $\tilde{P}(f)$, i.e. where $f \neq 0$, are normalized to have sums equal to before the addition of $R_{D}(f)$. This ensures that the total energy of the spectra are preserved, while the shape is changed. Figure 4.6 illustrates the Doppler residual in two skin models with different perfusion.
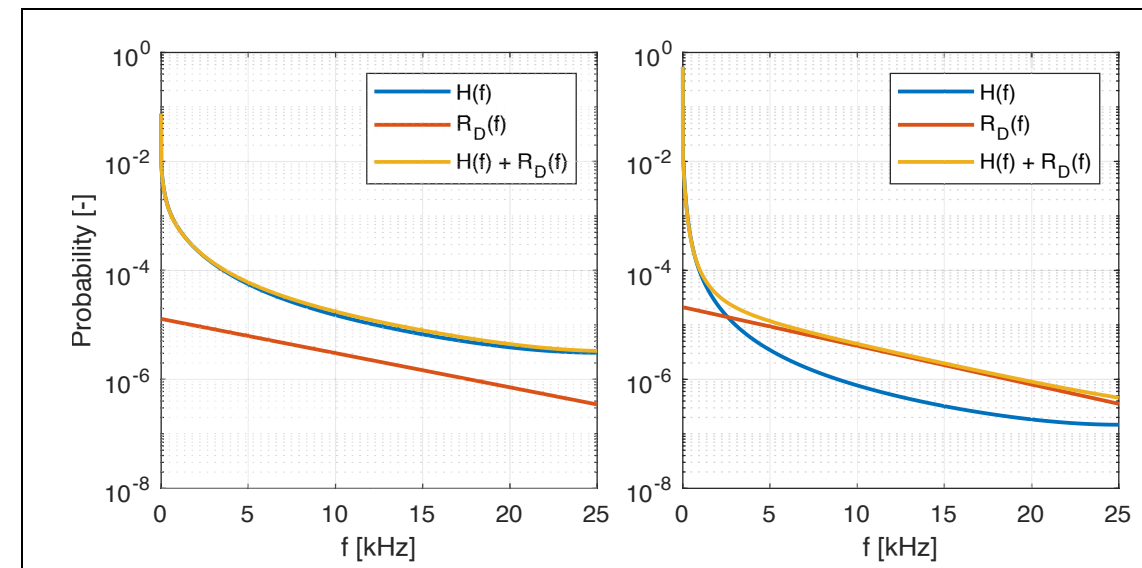

Figure 4.6: Illustration of the residual Doppler spectrum noise model in two skin models, where the residual spectrum has a low and high impact, respectively. The perfusion in the left model is 8.5 times higher than the right model. Also note that the residual spectrum in both plots are close to equal in magnitude, even though this might not appear to be the case at first glance.

\subsection{Speed-resolved perfusion}

Given the Doppler power spectrum, the conventional LDF perfusion can be computed as in Equation 3.3. However, while this perfusion estimate has a linear response to blood flow speeds, there is a non-linear response to blood concentrations [87]. In other words, this does not reflect the true perfusion in units $\% \mathrm{RBC} \times \mathrm{mm} / \mathrm{s}$. Instead, we compute the true perfusion from the speed-distribution of RBCs, as described partially in Ref [91]. To account for the contribution from each skin layer, we first compute the non-normalized 
sampling volume of light that is detected from each layer $m$, according to

$$
\varrho_{m}=\sum_{n=m}^{2}\left(I_{n} \int_{l} l p_{\mathrm{pl}, m, n}^{\prime \prime}(l) \mathrm{d} l\right)
$$

where the integral computes the expected absorption-modified path length, and $I_{n}$ is the absorption-modified detected intensity for all photons where the lowest layer reached was $n$ (see Equation 4.6). Normalizing $\varrho_{m}$ gives the sampling volume-fraction for each layer:

$$
\varrho_{m}^{\prime}=\frac{\varrho_{m}}{\sum_{j} \varrho_{j}} .
$$

Given the blood concentration $c_{\text {blood }}^{(m)}$ in skin layer $m$, the volume-fraction as computed above, and the speed distribution of RBCs, we can now compute the speed-resolved perfusion as

$$
P^{\left[v_{\min }, v_{\max }\right]}=\sum_{m=1}^{2}\left(\varrho_{m}^{\prime} \cdot 0.43 \cdot c_{\text {blood }}^{(m)}\right) \cdot \int_{v_{\min }}^{v_{\max }} v c_{\mathrm{RBC}}(v) \mathrm{d} v,
$$

where we assume a hematocrit of $43 \%$ [83]. We currently also assume the same speed-distribution in all layers. This can be extended to allow different speeddistributions in the dermis layers, but the integral must then be evaluated separately for each layer, and be included in the sum. Also note that the sum is excluding the epidermis layer, since this has $c_{\text {blood }}=0$.

By integrating the speed-distribution over different ranges of speeds $\left[v_{\min }, v_{\max }\right]$, speed-resolved perfusion is obtained, where each speed component only considers the perfusion from RBCs within that speed-range. In Paper VI, we present speed-resolved perfusion in three ranges, 0-1 mm/s, 1$10 \mathrm{~mm} / \mathrm{s}$, and $>10 \mathrm{~mm} / \mathrm{s}$, as well as the total perfusion given by the sum of all speed components.

\subsection{Solving the inverse problem}

So far, this chapter has presented the forward-calculations of the mathematical skin-model, i.e. the algorithm to go from skin properties to measured quantities. However, it is the inverse problem that is really of interest, where skin properties or related quantities are estimated from measured data. In most published works on the subject, this has been done by an iterative least-square optimization to fit the outputs from the forward calculation to the measured Doppler power spectrum [83] or the diffuse reflectance absorption spectrum $[83,85]$. This gives a high degree of control over the allowed solutions, and it enables direct insight into why the optimal solution is found. Unfortunately it is also slow, to the point where no realistic real-time implementation is 
likely for imaging applications. For the purposes of this thesis, which aims to compute real-time perfusion images from the MELSCI data, this method is therefore not viable. Instead, machine learning has been used to learn a transfer function, in the form of an artificial neural network (ANN), from multi-exposure contrast $K^{2}(T)$, directly to either conventional LDF perfusion $P_{\mathrm{LDF}}\left[\right.$ Paper III] or speed-resolved perfusion $\left\{P^{[0,1]}, P^{[1,10]}, P^{[>10]}\right\}[\mathrm{Pa}-$ per VI]. Similar ANN-based solutions have been proposed for estimating skin properties and blood oxygen saturation from the diffuse reflectance spectrum $[84,92]$.

While the details of how to train a neural network is left outside the scope of this thesis, there are some important considerations when using modeled multi-exposure contrast in machine learning. One of the main results of $\mathrm{Pa}-$ per III is a noise model for adding so called speckle realization noise to the contrast. Speckles is a statistical process, and the exact value of the contrast depends on the specific phases of the electric fields at the time of the measurement. This creates a noise in the contrast that is only possible to remove by averaging, either spatially or temporally. The contrast from the model in this chapter does not contain this noise, which results in an ANN that does well on simulated data, but completely fails to generalize to real data. We therefore developed a model to add the speckle realization noise to the modeled contrast, based on the noise characteristics from speckle simulations in 2000 skin models. The noise model is valid for the exposure times $1,2,4,8$, 16,32 , and $64 \mathrm{~ms}$, which is used in the MELSCI-implementation described in the next chapter.

The essence of the noise model is that the noise level at exposure time $T$ depends on two factors: the average contrast over all exposure times, $\langle K(T)\rangle_{T}$, and the value of the contrast at one exposure time lower, which in this implementation is $T / 2$. Thus, the noise model was defined as

$$
\begin{aligned}
K_{\text {noise }}(T) & =\langle K(T)\rangle_{T} \\
& \times \begin{cases}\xi_{T} \eta_{1} & \text { for } T=1 \\
K_{\text {noise }}\left(\frac{T}{2}\right)+\xi_{T} \eta_{\text {diff }}(T) & \text { for } T \in[2,4, \ldots, 64],\end{cases}
\end{aligned}
$$

where the constants $\eta$ were determined from the speckle simulations, and $\xi_{T} \in \mathcal{N}(0,1)$ are random scalars drawn from a normal distribution with mean 0 and standard deviation 1 . In simpler terms, $K_{\text {noise }}(1 \mathrm{~ms})$ is first determined, then each successive $K_{\text {noise }}(T)$ is determined based on a random change from the preceding noise level. In the final step, the noise vector is added to the modeled contrast. Figure 4.7 shows how the noise model successfully captures the noise behaviour in the speckle simulations. The noise-modified contrast was used to train the ANNs in Paper III and Paper VI, with good generalization to real data as a result. 


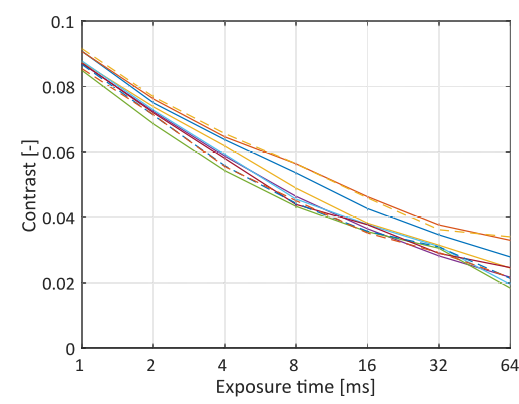

(a) From speckle simulations

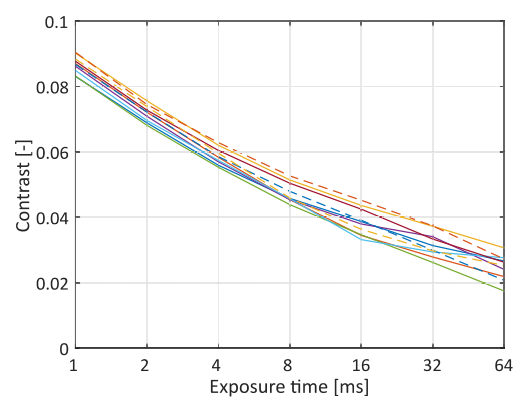

(b) From noise model

Figure 4.7: (a) 10 contrast curves from speckle simulations of the same tissue model, where the differences are due to the unique realization of the speckle pattern during the exposure window. (b) 10 contrast curves created by adding the noise model to the average of the curves in (a). The low contrast values are because the noise model was applied before the normalization with the maximum contrast. Figure recreated from Paper III with permission. 


\section{5}

\section{Design and implementation of a perfusion imaging instrument}

A large portion of this project has been spent on the design, implementation, validation, and operation of a MELSCI-based perfusion imaging instrument. The core algorithms are presented in Paper I, IV, and VI, but this chapter is a more detailed description of the instrument design, from the FPGAalgorithms to the user interface designed for the clinical studies.

\subsection{System overview}

The core of the system is based on the principle of synthetic multi-exposure, first presented by Dragojević et al. [21], where single-exposure speckle images from a high-speed camera is digitally accumulated to create longer exposure times. To achieve real-time continuous processing, the implementation was done in a field-programmable gate array (FPGA). This is a computation device where a very large number of simple configurable logic blocks (CLBs) are combined by a programmable network of connections to perform complicated tasks. Each CLB is capable of performing simple operations such as binary addition and carry logic, signal multiplexing, look-up-tables, and storage in flip-flops or latches. In addition to the CLBs, the FPGA also contains resources for distributed on-chip memory, which provides extremely fast working memory for the implemented algorithms. The computational power of the FPGA comes from the fact that (essentially) all configured operations are performed in parallel, once every clock cycle. With typical clock speeds in the hundreds of $\mathrm{MHz}$, and several hundred thousand parallel CLBs, the possible number of operations per second is staggering. While this project has sometimes involved design considerations on the low level of single CLBs, most often this is abstracted away by the modern design tools. These fine details are also beyond the scope of this chapter, which will instead present a high-level overview of the implemented design.

The design presented in this chapter is the same that was presented in Paper IV, then used in Papers V and VI. Before that, another system was used, as presented in Papers I-III. However, this design was discarded in 
favour of the current one due to limitations which prevented the fully realized solution that is the result of this thesis (see Chapter 6 for more details). Figure 5.1 presents a schematic overview of the implemented system, which will be explained in this chapter.

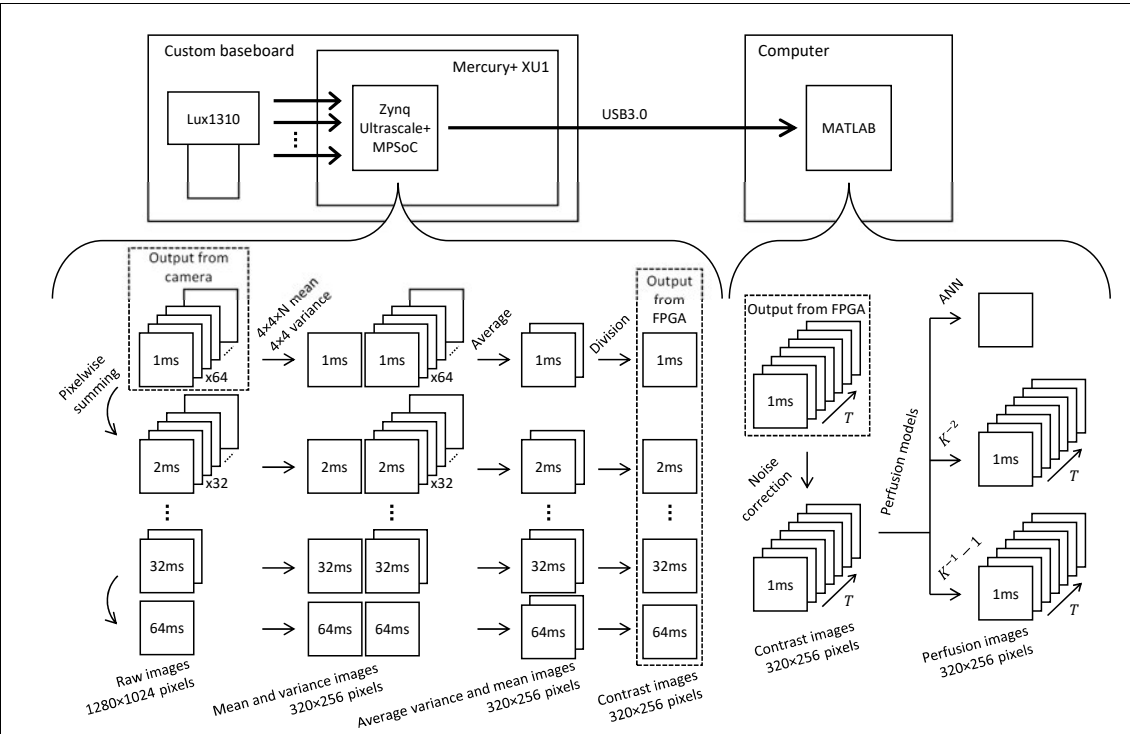

Figure 5.1: Overview of the system and algorithms. Each part will be explained in the following sections. Reprinted from Paper IV with permission.

The main requirement for computing synthetic exposure times is that speckle images are captured with a negligible inter-frame delay. This allows longer exposure times to be created by digitally adding two consecutive images pixel-wise. Thompson et al. [20] demonstrated that with a sufficiently wide range of exposure times, the Doppler power spectrum could be computed from multi-exposure contrast, essentially making the modalities equivalent measurements. However, they used a shortest exposure time of $50 \mu \mathrm{s}$, which in this implementation would restrict the size of the images due to limitations in the camera sensor. We therefore use a shortest exposure time of $1 \mathrm{~ms}$, obtained from a 1000 frames-per-second (fps) sensor with an inter-frame delay of $2 \mu \mathrm{s}$. Technically, this means that the exposure time is $998 \mu \mathrm{s}$, but for convenience $\mathrm{I}$ will refer to this as $1 \mathrm{~ms}$. With a bit-depth of 12 , and a size of $1024 \times 1280$ pixels, continuous read-out results in a throughput requirement of $1024 \times 1280 \times 12 \times 1000=15.7 \mathrm{Gbps}$. This is too fast to transfer over a single data channel, and the sensor interface therefore requires 16 parallel differential channels, each operating at a rate of approximately 1 Gbps. 
The high throughput is also the main reason why an FPGA implementation of the algorithm is required. This system used the Xilinx Zynq Ultrascale+ MPSoC, a multi-purpose system-on-chip with an FPGA and a quad-core 64-bit ARM CPU. This allows fast implementation of the synthetic exposure time algorithm in the FPGA, while the CPU handles the communication and data transfer to the computer. To connect the high-speed sensor to the FPGA, a custom base-board was designed, as seen in Figure 5.2. Features such as an SD-card reader to store the FPGA configuration and software, and a USB 3.0 output port for transferring the processed data to the computer, were also added.

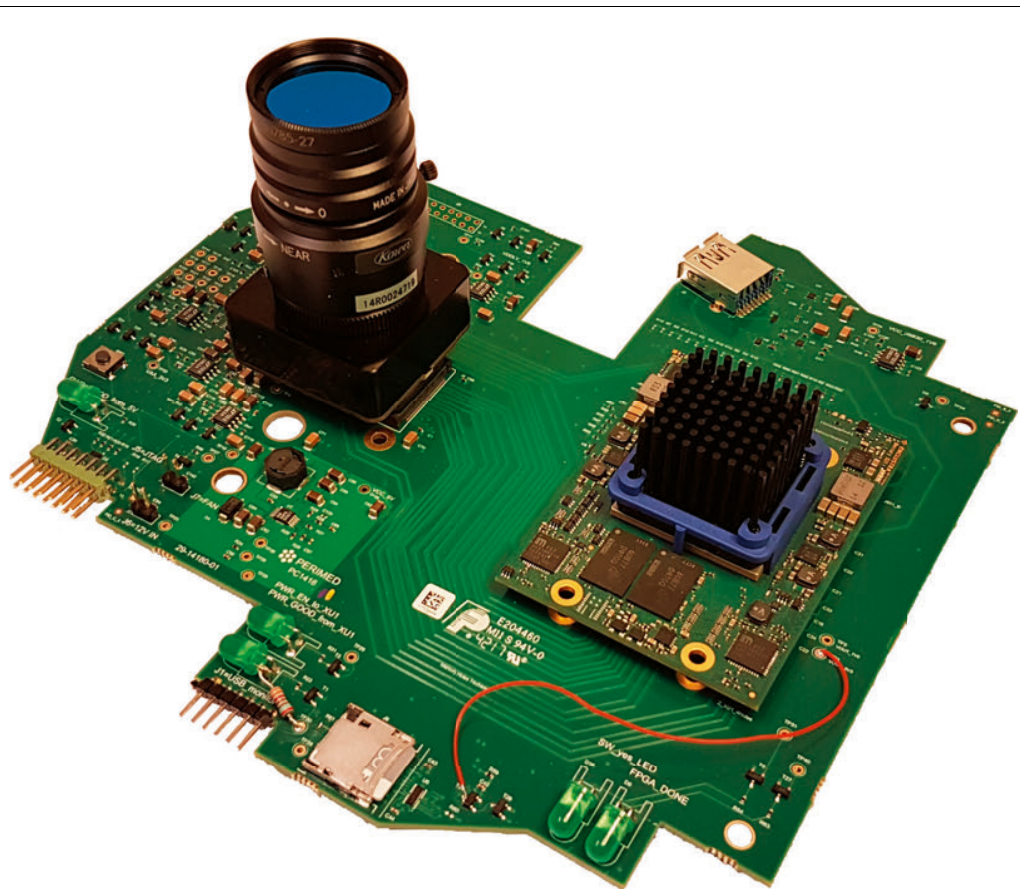

Figure 5.2: Photo of the custom PCB designed to connect the camera sensor to the FPGA. The parallel data channels can be seen between the two devices.

\subsection{Synthetic exposure time algorithm}

The synthetic multi-exposure algorithm is presented in Paper I and Paper IV, but will be explained here as well, with some additional details. The camera collects $T=1 \mathrm{~ms}$ speckle images with a negligible inter-frame delay of $2 \mu \mathrm{s}$. After $64 \mathrm{~ms}$, a set of 64 images have therefore been collected. This set is 
processed in a binary tree structure, where each pair of images are added to create 32 new frames with an exposure time of $T=2 \mathrm{~ms}$. Pairs of these are then added to create 16 frames with $T=4 \mathrm{~ms}$ exposure time. This continues until all images have been added, resulting in a single frame with $T=64 \mathrm{~ms}$ exposure time. This process is illustrated in Figure 5.3, and is represented by the first column in Figure 5.1.

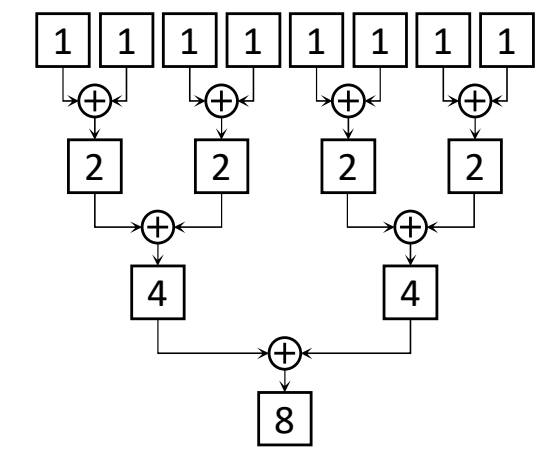

Figure 5.3: Illustration of the binary tree accumulation algorithm for computing synthetic exposure times. The squares represent individual frames, with the number indicating the exposure time. For clarity, this illustrates using 8 frames of $1 \mathrm{~ms}$ instead of 64 . The tree structure naturally expands to the 64 frames used in the presented implementation.

The binary tree accumulation results in 7 different exposure times $T \in[1,2,4,8,16,32,64] \mathrm{ms}$, with $N=64 / T$ unique images for exposure time $T$. This gives a total of $\sum_{k=0}^{6} 64 / 2^{k}=127$ images. For each of these, the variance in every non-overlapping $4 \times 4$ pixel region is computed, followed by averaging the $N$ images for each exposure time, to reduce speckle realization noise and to utilize all available information. This gives 7 variance images at the powers of 2 exposure times. The variance calculation is summarized in the following equations,

$$
\begin{gathered}
i_{x, y, n}(T)=\sum_{t=1}^{T} i_{x, y, t, n} \\
\sigma(T)=\underbrace{\frac{1}{N} \sum_{n=1}^{N}}_{\text {Noise reduction }} \underbrace{\frac{1}{4^{2}}\left[\left(\sum_{y=1}^{4} \sum_{x=1}^{4} i_{x, y, n}^{2}(T)\right)-\frac{1}{4^{2}}\left(\sum_{y=1}^{4} \sum_{x=1}^{4} i_{x, y, n}(T)\right)^{2}\right]}_{4 \times 4 \text { variance kernel }},
\end{gathered}
$$

where the synthetic exposure time is first computed in (a), where $i_{x, y, t, n}$ denotes the individual pixel intensities for position $x, y$ in the $4 \times 4$ block, and the sum over $t$ is the exposure time accumulation. This is then used in the one-pass variance calculation in (b), and finally the averaging over the $N$ different variance images is applied. Note that the one-pass variance calculation is valid here since this is implemented in integer arithmetic in the FPGA, 
with the bit-widths expanding as needed to contain the theoretical maximum sums and squares (the largest value is represented by a 40-bit integer). This implementation therefore does not suffer from the effects of catastrophic cancellation seen in floating-point one-pass implementations [93].

The average intensity is also computed in the corresponding $4 \times 4$ pixel regions, then averaged in the same way as the variance images. Using the definition in Equation 5.1a, this operation is defined as

$$
\langle I(T)\rangle=\underbrace{\frac{1}{N} \sum_{n=1}^{N}}_{\text {Noise reduction }} \overbrace{\frac{1}{4^{2}} \sum_{y=1}^{4} \sum_{x=1}^{4}}^{4 \times 4} i_{x, y, n}(T),
$$

where $\langle I(T)\rangle$ is the final noise-reduced intensity image for exposure time $T$. But since $N=64 / T$, the only difference between $\langle I(T)\rangle$ for different exposure times is a scaling by $T$. Consequently, if we define $\langle I\rangle$ to be the average intensity of the 64 captured images, which in this implementation have an exposure time of $1 \mathrm{~ms}$, we have

$$
\langle I(T)\rangle=T\langle I\rangle
$$

This means that the average intensity image only has to be computed for a single exposure time, and the rest are given by the scaling. Columns 2 and 3 in Figure 5.1 illustrate these steps.

The final step is to divide the variance by the squared intensity to get the squared contrast, represented by column 4 in Figure 5.1. While division in fixed-point arithmetic is possible, we instead translate the variance and intensity to 32-bit floating point representation, using built in primitives in the FPGA. The division is then made, and the contrast images saved to external memory to be transferred to the computer. Due to the size reduction by the $4 \times 4$ kernel, a single multi-exposure contrast cube has size $256 \times 320 \times 8$ of 32-bit floats, where the 8 is from 7 exposure times plus the average intensity image. This is a data reduction from $15.7 \mathrm{Gbps}$ to $328 \mathrm{Mbps}$, i.e. 48 times. The framerate of the processed contrast images is decided by the set-size of the synthetic multi-exposures (64 frames), and the base framerate (1000 fps), since no frames are shared between consecutive multi-exposure contrast cubes. This gives a framerate of $1000 / 64=15.625 \mathrm{fps}$, which is sufficient to detect fast dynamics in the blood flow.

\subsection{Other implementation details}

Two other implementation details are particularly important for the results of this thesis - the flow control and the data buffering strategy. Without these, the continuous measurements and real-time processing would not have 
been possible. However, while important, these details are not necessary to understand the results of the thesis. For that reason, I will keep this explanation short.

\section{Flow control}

Images are always collected in sets of 64 , which are sent to the rest of the algorithm. As part of the collection process, the images go through a flowcontrol unit implemented in the FPGA. The purpose of this is to control the flow of images to the rest of the system, in the unlikely event of delays further down the line. Most commonly, this delay happens on the computer, which for various reasons might not keep up with the stream of processed images. The system was designed to have a considerable buffer space to be able to store processed images until the computer is ready to receive them. However, if the buffer fills due to an extended delay, a solution is needed.

One possibility is simply to discard any images that cannot be buffered, but this might cause a large gap in the measurement where no images are recorded. A better solution is a soft decrease in the framerate of the contrast images, to gradually lower the rate at which the buffer fills. This ensures that even in the event of a long delay, images are still captured and processed, although not as fast as the ideal case.

The naive way to lower the framerate would be to not request new images from the sensor. However, the sensor accumulates dark noise even during the down-time, which would make the noise correction invalid for the first image after the pause. To avoid this problem, the flow-control is instead handled entirely in the FPGA, by discarding a set number of $1 \mathrm{~ms}$ frames between each set of 64 frames. This ensures that all frames passed to the rest of the algorithm have the same noise levels, and that each set is from 64 consecutive frames. The number of discarded frames between each set is

$$
n_{\text {discard }}=r \cdot \max \left(0, n_{\text {buff }}-\left\lfloor f N_{\text {buff }}\right\rfloor\right),
$$

where $N_{\text {buff }}$ is the size of the image buffer, $n_{\text {buff }}$ is the current number of buffered images, $f \in[0,1]$ is the fraction of the buffer that can be filled without discarding any frames (i.e. the soft-limit of the buffer), and $r \geq 1$ is an integer scale factor controlling the rate of slowdown once the soft-limit is exceeded. This function is reminiscent of a hinge loss used in some machine learning applications, in the sense that it has a portion where "errors" (buffered images) are allowed, after which the "loss" (discarded images) grows linearly with the error.

In an ideal world, the flow control block would not be required, and future software optimization might indeed make it obsolete. However, for the current system it was a necessity to enable continuous measurements over long times. This was especially true for the data collection in Paper V, where measure- 
ments were often several hours long, and the system could not be monitored at all times.

\section{Data buffering}

A full set of 64 images, which totals $1024 \times 1280 \times 12 \times 64=126$ MB of data, is unfortunately too large to be kept in the internal distributed memory on the FPGA (i.e. the fast memory). Data must therefore be buffered in an external memory, then later retrieved in smaller chunks for the calculations. The memory used here is a 2 GB DDR4 SDRAM with a transfer speed of 19.2 GB/s, which is more than sufficient. In general in the design, first-infirst-out ring-buffers are used to transfer data from one subsystem to another, both in the internal and external memories. By dividing each buffer space into at least two sub-buffers, which can only be accessed by a single subsystem at a time, data can be both written and read from the buffer at the same time, effectively doubling the performance. This was a requirement to enable continuous processing of images without discarding data. The sub-buffers in the external memory are each the full size of 64 frames (i.e. $128 \mathrm{MB}$ ), but this varies in each subsystem with some sub-buffers as small as $8 \mathrm{kB}$. For the same reason that the flow control was implemented, some ring-buffers contain more than two sub-buffers. This ensures smooth data flows even in the event of downstream delays. An illustration of a double-buffered and quad-buffered memory is presented in Figure 5.4.

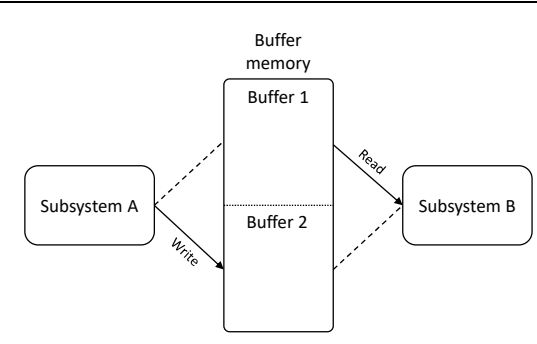

(a) Double buffered memory.

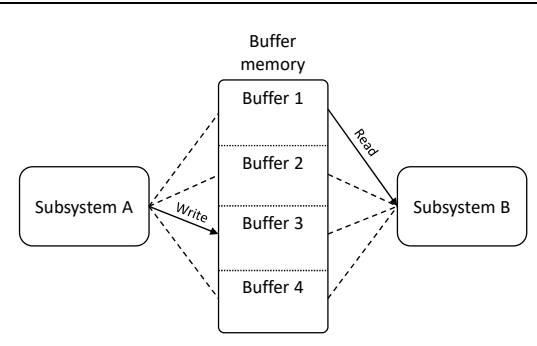

(b) Quad-buffered memory.

Figure 5.4: Illustration of different levels of ring-buffering used to allow a continuous data flow even in the presence of delays in some part of the system. The read-head and write-head are never allowed in the same buffer region, to prevent race conditions. 


\subsection{Embedded software layer}

A custom Linux operating system was designed for the ARM CPU, enabling the use of powerful software libraries for the data transfer to the computer. The data transfer was implemented using Ethernet over USB, to allow the use of the TCP or UDP protocol. This makes interfacing easy with virtually any programming language, including Matlab which was used to build the prototype user interface for the instrument. At the full framerate, the stream of contrast images requires a throughput of $256 \times 320 \times 8 \times 32 \times 15.625=328 \mathrm{Mbps}$. Theoretically, USB 3.0 supports transfer speeds of 5 Gbps. However, the Ethernet over USB implementation introduces severe overheads that limits the transfer speeds to approximately $1.3 \mathrm{Gbps}$ in the case of TCP, and $2.0 \mathrm{Gbps}$ in the case of UDP. Since this is still above the required speeds with a wide margin, no further efforts were spent on better solutions.

\subsection{User interface}

To facilitate easy data collection, as well as live presentation of perfusion images, a prototype user interface was developed in Matlab App Designer. The artificial neural network trained in Paper III was implemented to estimate the perfusion in real-time, as seen in the final column of Figure 5.1. The contrast images were saved to a configurable location, with any metadata required for post-processing. A minimal comment functionality was implemented, where time-tagged text comments could be stored together with the images. This was especially useful in the data collection for Paper V. Extensive debugging features were also added, which were very useful in the early stages of the project. Figure 5.5 illustrates the UI when used in an occlusion-release experiment of a finger. 


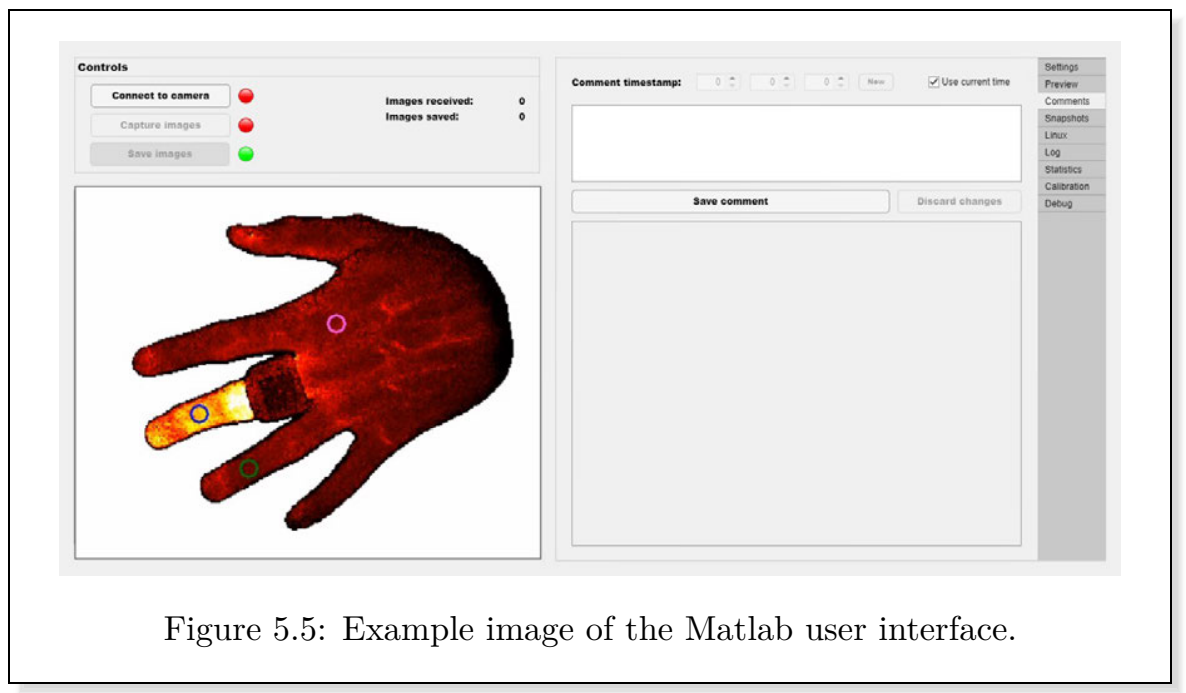


5. DESIGN AND IMPLEMENTATION OF A PERFUSION IMAGING INSTRUMENT 


\section{6}

\section{Review of papers}

The six papers in this thesis present a step-by-step solution to a MELSCIbased imaging system for continuous real-time skin perfusion measurements in more accurate and physiologically relevant units. In Paper I, we presented the implementation of a fast algorithm for MELSCI in a field-programmable gate array (FPGA). The results demonstrated that a real-time implementation of MELSCI using synthetic exposure times was possible, but the performance was limited by the slow transfer of processed data to the computer. In Paper II, we evaluated an existing analytical model for MELSCI-based perfusion (Equation 3.21) using data collected with the system from Paper I. The model displayed a small but systematic error, indicating that it could not capture all aspects of the data. Additionally, fitting the model to the data was extremely time-consuming, and therefore impossible to use in real-time. In Paper III, we addressed this by presenting an artificial neural network (ANN) perfusion model trained on a large dataset of simulated measurements. We demonstrated that the ANN generalized to in-vivo data, and compared it to existing analytical perfusion models for LSCI and MELSCI. The ANN was superior in terms of accuracy and computational speed, enabling real-time computation of perfusion images with quality comparable to LDF. In Paper IV, we connected the results from Paper I and Paper III by presenting a MELSCI instrument capable of continuous real-time perfusion imaging at framerates sufficient to resolve the fastest blood flow dynamics in the microcirculation, while retaining the high accuracy of the ANN perfusion estimate.

In Paper V, we used the system from Paper IV to investigate the microcirculation dynamics in the foot of patients undergoing revascularization surgery due to chronic limb-threatening ischemia. Multi-spectral imaging was also used to enable a comprehensive evaluation of the microcirculation. We demonstrated that expected changes in perfusion and oxygen saturation could be observed in the data, but also discovered unexpected dynamics that - with more research - could lead to new and improved clinical practices based on the microcirculation.

Finally, in Paper VI we presented an ANN for estimating speed-resolved perfusion in absolute units from MELSCI data. While the perfusion ANN from Paper III was more accurate than previous analytical models, it was still 
limited to a single perfusion estimate in relative units. To further facilitate the physiological interpretation of the data, the ANN train in this paper instead estimated perfusion in three distinct speed-ranges. Together with the absolute unit $\% \mathrm{RBC} \times \mathrm{mm} / \mathrm{s}$, this could potentially increase the clinical value of the measurements.

The rest of this chapter will present the papers in more detail, will highlight of some of the most important results, and will discuss some of the potential issues.

\subsection{Paper I}

In this paper, titled $A$ 15.6 frames per second 1-megapixel multiple exposure laser speckle contrast imaging setup, the aim was to show a working FPGAbased implementation of MELSCI that could run in real-time without any loss of data. The implementation was based on synthetic multi-exposure, as introduced by Dragojević et.al. [21]. This algorithm was covered in the previous chapter, Section 5.2.

The results showed that multi-exposure contrast images computed by this system decreased as expected with increasing exposure time. The contrast also displayed the expected increase and subsequent decrease when evaluated in a standardized arterial occlusion of a finger, while the contrast in the non-provoked tissue remained almost unchanged by the experiment. Multiexposure contrast images during the reperfusion are presented in Figure 6.1. The results also highlighted the high quality of data in both images and individual multi-exposure contrast curves. We concluded that this was due to the optimal use of the available data, where all 64 frames were used to compute each exposure time, as described in the previous chapter.

There were some problems with the design. Specifically, the data transfer to the computer was controlled by a soft micro-processor created in the FPGA logic. While this was a highly flexible solution, the performance was not sufficient to transfer every multi-exposure frame to the computer. This meant that, while the FPGA implementation was real-time at 15.6 contrast images per second, the actual output to the computer was only $1.5 \mathrm{fps}$, far too low to resolve the temporal dynamics of the blood flow. We concluded that the best solution to this problem would be to move the implementation from a pure FPGA to a system-on-chip with both an FPGA and a CPU. This would eventually be realized in Paper IV. 


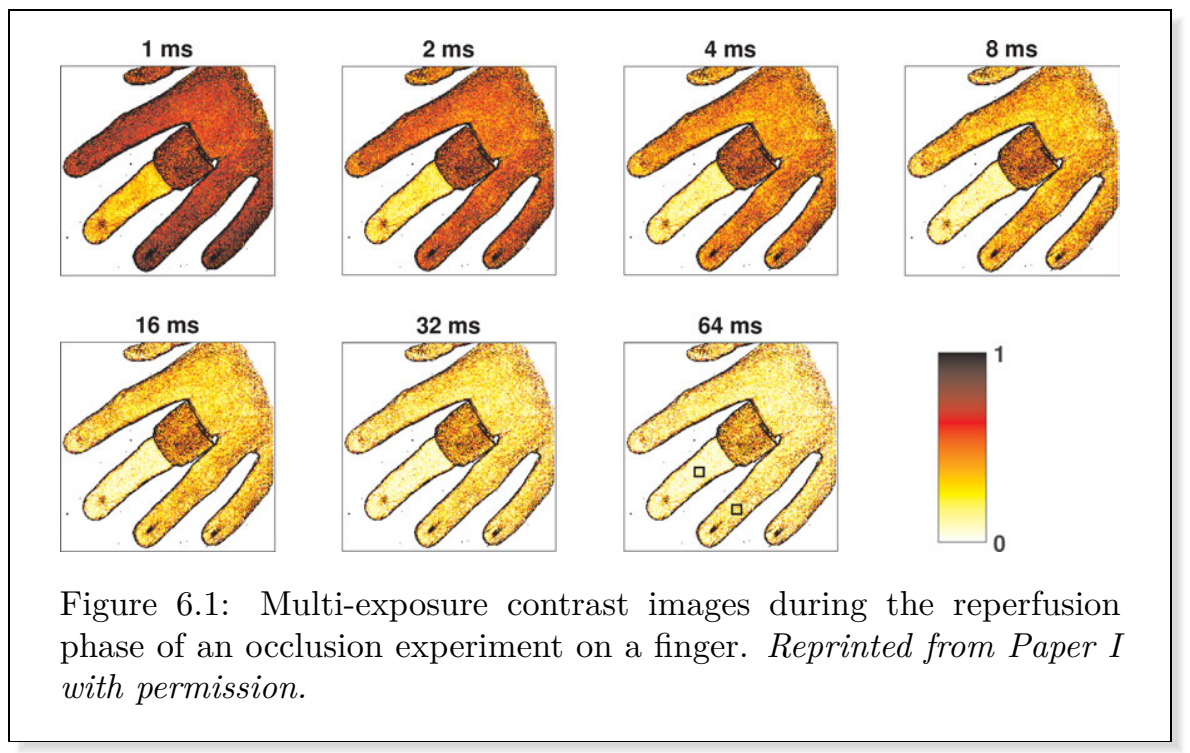

\subsection{Paper II}

In the second paper, titled Evaluation of a high framerate multi-exposure laser speckle contrast imaging setup, we used the system presented in Paper I with an analytic MELSCI perfusion model by proposed by Kazmi et.al. [39] (Equation. 3.21) and compared it to perfusion from a single-point LDF system. Additionally, we compared this to perfusion by single-exposure LSCI, computed using the 4-ms images of the MELSCI data. The comparisons were made on data from a standardized arterial occlusion of the forearm in a healthy volunteer.

The multi-exposure contrast model was fit to the data and, once the optimal fit was found, the model parameters were used to compute perfusion according to Equation 3.22b. Images from the occlusion-release experiment presented in Paper I were also analyzed, and are reprinted here in Figure 6.2. As expected, the perfusion estimate from MELSCI was superior to that of LSCI in terms of linearity to LDF, but it also demonstrated higher variance in the perfusion estimate, as seen in Figure 6.3. We found that the model could fit the data well, although there were consistent deviations in the model fit that indicated that some aspects of the data were not captured by the model. This was further evidenced by the inability of the model to fit the data if we fixed the model parameter $\beta=1$. In theory, this should have had no effect on the results since the contrast images had been calibrated according to Section 3.4 and 3.5. However, we found that this increased the average model error by several orders of magnitude since the model failed to converge on a solution in a large portion of the data. While this alone could have been 
explained by a poor calibration, we also observed that when leaving $\beta$ as a free model parameter, the optimal value changed when occluding the arm. The purpose of $\beta$ is an instrument correction constant, and should not be influenced by the tissue status, again indicating that the model could not fully explain the data. In addition to these problems, the model fitting was too slow to be implemented in real-time, requiring over 4 minutes per frame, and we concluded that the most likely solution would be to find a new method to estimate the perfusion, that did not rely on model fitting.
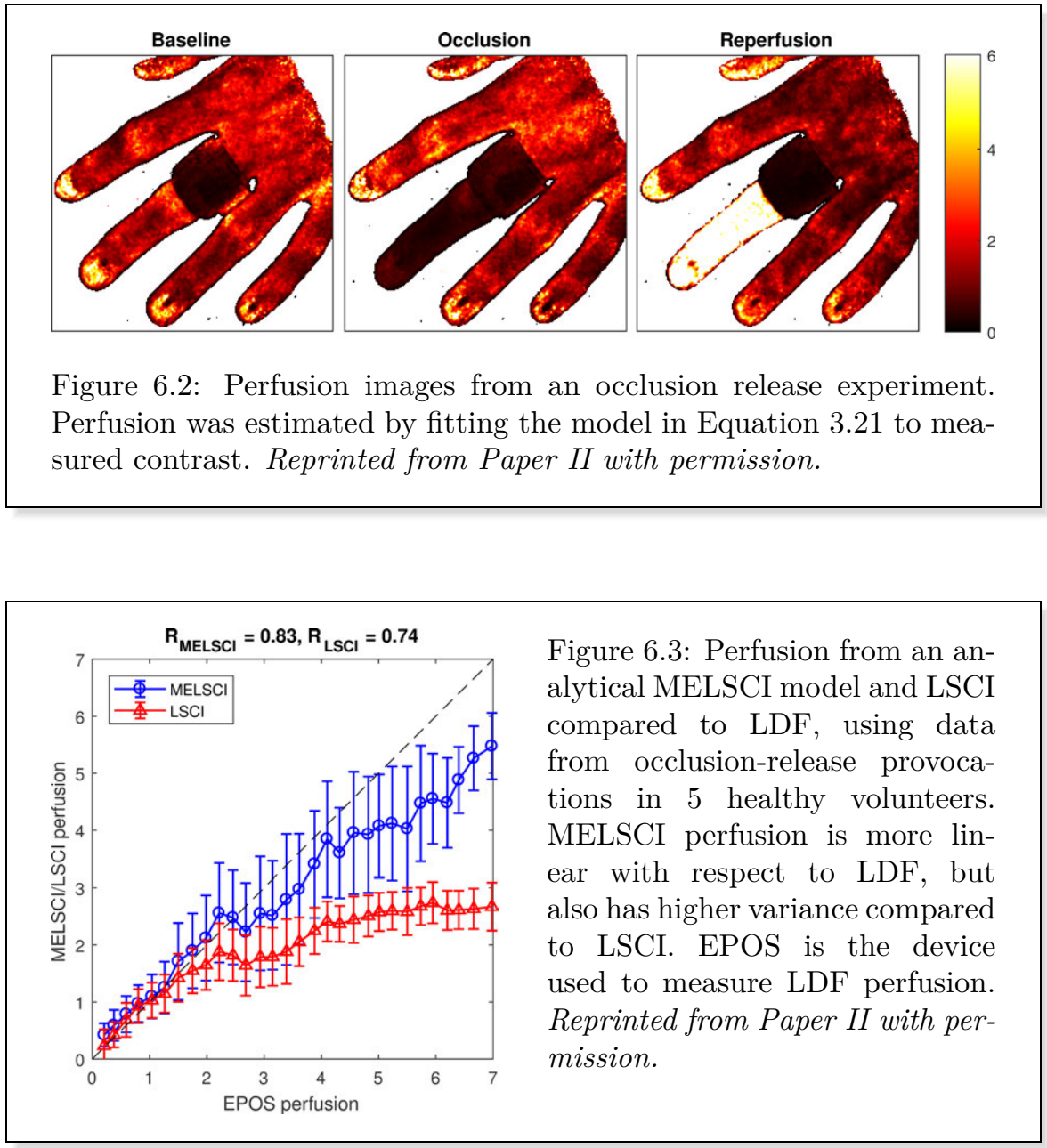


\subsection{Paper III}

In this paper, titled Machine learning in multiexposure laser speckle contrast imaging can replace conventional laser Doppler flowmetry, we proposed a method for generating realistic multi-exposure contrast data from a mathematical skin model, and demonstrated how an ANN could be trained on this data to estimate conventional LDF perfusion. Unlike the model used in Paper II, which was based on fitting an analytical model to the data, the ANN directly estimated perfusion from multi-exposure contrast, offloading the slow part of the algorithm from run-time to the training stage. Additionally, the ANN demonstrated higher accuracy and less susceptibility to measurement noise compared to the analytical perfusion model from Paper II.

The training dataset was created using the mathematical framework described in Chapter 4. A dataset of 100000 skin tissue models was created and used to train the ANN, which was a simple fully-connected network with one hidden layer with ten nodes. With seven inputs (multi-exposure contrast) and one output (LDF perfusion, Equation 3.3), the total number of parameters in the network was only 91 , but we found no significant performance increase when training a larger model. This indicated that the problem of translating multi-exposure contrast to LDF perfusion is actually rather simple. It is therefore reasonable to assume that the increased accuracy is a result of highly informative learned features, rather than the computational power of the model.

An important aspect of the training process was the inclusion of a noisemodel for the multi-exposure contrast, which was described in Section 4.7. To briefly summarize, the simulated contrast was noise-free, resulting in extremely poor performance on in-vivo data. This was expected, since there are noise sources in real data that are not captured by the computational model. Thus, a noise-model was developed to make the simulated data more realistic by adding the so called speckle realization noise. The noise model was applied to the simulated data, allowing the ANN to compensate for these variations. We demonstrated that underestimating the amount of speckle realization noise severely reduced the performance, while overestimating it did not.

Four perfusion models were compared against LDF perfusion using a simulated dataset of 50000 skin models. The accuracy of the perfusion models is presented in Figure 6.4. The LSCI perfusion had a non-linear relation to LDF perfusion, with high standard deviation in the predictions (Figure 6.4a), while the analytical MELSCI perfusion model was more linear and had lower standard deviation (Figure 6.4b). The ANN outperformed both analytical models, whether trained with or without speckle realization noise. As seen in Figure $6.4 \mathrm{c}$, the noise-free ANN had close to ideal performance $(R=1.000)$. However, only the ANN trained with speckle realization noise, Figure $6.4 \mathrm{~d}$, 
performed well on in-vivo data. This is the ANN that was used in Papers IV and $\mathrm{V}$.

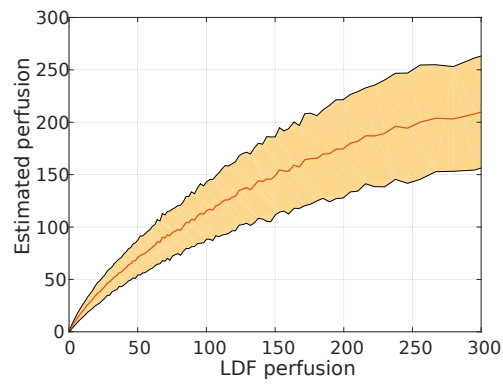

(a) $6 \mathrm{~ms}$ LSCI model

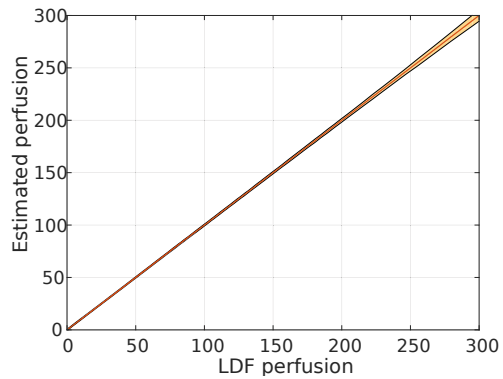

(c) ANN (without noise)

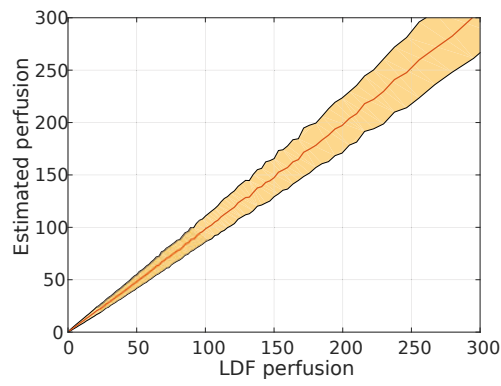

(b) Analytical MELSCI model

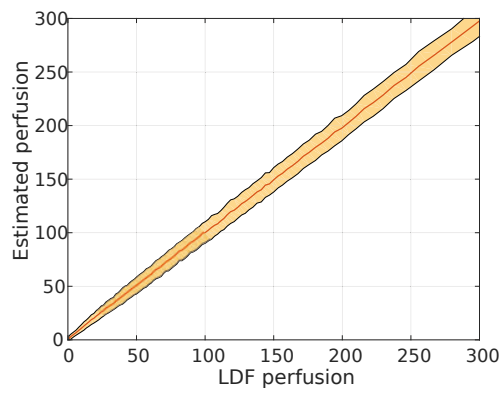

(d) ANN (with noise)

Figure 6.4: Perfusion models compared to conventional LDF perfusion. The shaded area indicates the standard deviation in predicted perfusion. (a) Single-exposure LSCI at $6 \mathrm{~ms}$ (see Equation 3.19, $n=1$ ). (b) Analytical MELSCI perfusion (see Equation 3.21). (c) ANN trained and evaluated without speckle realization noise. (d) ANN trained and evaluated with speckle realization noise, i.e. the ANN later used for in-vivo data. Recreated from Paper III with permission.

The ANN was applied on in-vivo MELSCI data in two experiments. First, the ANN perfusion was compared to conventional LDF perfusion during an arterial occlusion provocation of the forearm of a healthy volunteer. This was measured with a $20 \mathrm{kHz}$ camera, which enabled LDF and MELSCI processing of the same data, making the comparison more meaningfully than when using separate measurement systems. The correlation between the perfusion by LDF and by the ANN was high $(R=0.995)$, although the ANN slightly underestimated the perfusion compared to LDF during the post-occlusion peak, indicating that the model did not perfectly capture the behaviour of LDF. Second, we performed an arterial occlusion provocation of a finger, 
this time measuring with a 1000 fps camera as presented previously in this thesis. This demonstrated the high visual quality in the perfusion images, which are presented in Figure 6.5. Additionally, we used this experiment to measure the computational performance of the model, i.e. the time to compute one perfusion image from one multi-exposure contrast frame. This time was measured to be $28 \mathrm{~ms}$ per image, significantly shorter than the 64 ms required to collect the corresponding data. In other words, the ANN not only outperformed previous perfusion models for LSCI and MELSCI, it also enabled real-time perfusion imaging from MELSCI, which was one of the main goals of the study.

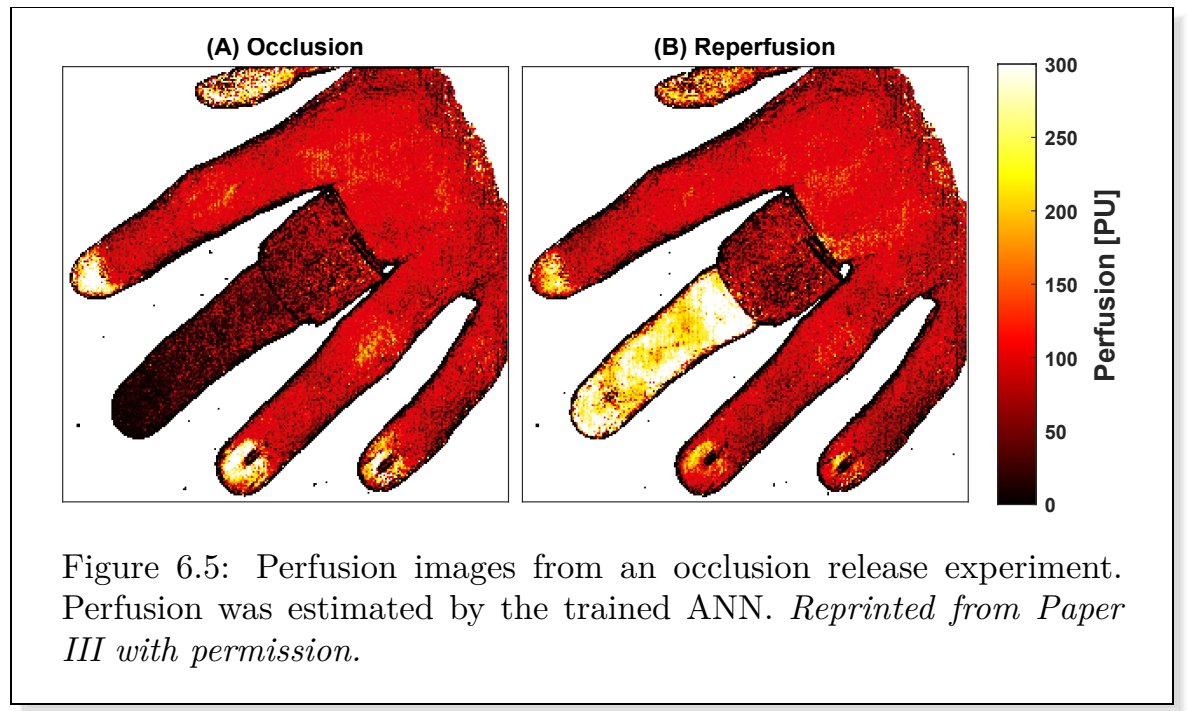

\subsection{Paper IV}

In the fourth paper, titled Real-time video-rate perfusion imaging using multiexposure laser speckle contrast imaging and machine learning, we combined the multi-exposure contrast algorithm from Paper I and the ANN perfusion model from Paper III, demonstrating a MELSCI-based system for real-time skin perfusion imaging. The system was essentially the same as in Paper I, but implemented on a custom platform with both FPGA and multi-core CPU capabilities, as described in Chapter 5. This combined system-on-chip (SoC) enabled efficient implementation of the contrast algorithm on FPGA and the data transmission logic in the CPU, solving the bottleneck problems discussed in Paper I. Together with the real-time implementation of the ANN from Paper III, this system enabled continuous MELSCI-based perfusion imaging 
at high framerates in real-time, with perfusion accuracy comparable to that of LDF. This result was the first major goal of this thesis.

We evaluated the system in two experiments. First, we compared the perfusion estimate from the proposed system to perfusion by LDF, using a beaker of milk that was heated and cooled to different temperatures, changing the speed of particles in the liquid due to Brownian motion, which in turn changed the perfusion. This allowed us to measure a wide range of perfusion values and compare the two methods. Results confirmed the high correlation between ANN perfusion and LDF perfusion seen in Paper III; $R=0.996$ in the new experiment.

The second experiment was once again an arterial occlusion of the forearm of a healthy volunteer, measuring 5 minutes each of baseline, occlusion, and reperfusion. We compared the ANN perfusion estimate to two common singleexposure perfusion models, first in a region of interest and then using images from the three phases of the provocation. The lack of a ground truth perfusion meant that no quantitative conclusions could be drawn in this experiment. However, the ANN perfusion displayed a much larger dynamic range, i.e. lower during occlusion and higher during reperfusion, as well as less influence by noise and a more pronounced heart-beat signal. The images also indicated that the ANN perfusion might be more sensitive to flow from deeper vessels than LSCI perfusion, possibly because the ANN is more sensitive to light that has been multiple shifted, although this should be further investigated in future research. Regardless, it was apparent that the increased information available in the multiple exposure times was beneficial. Figure 6.6 presents the perfusion images from the experiment. 


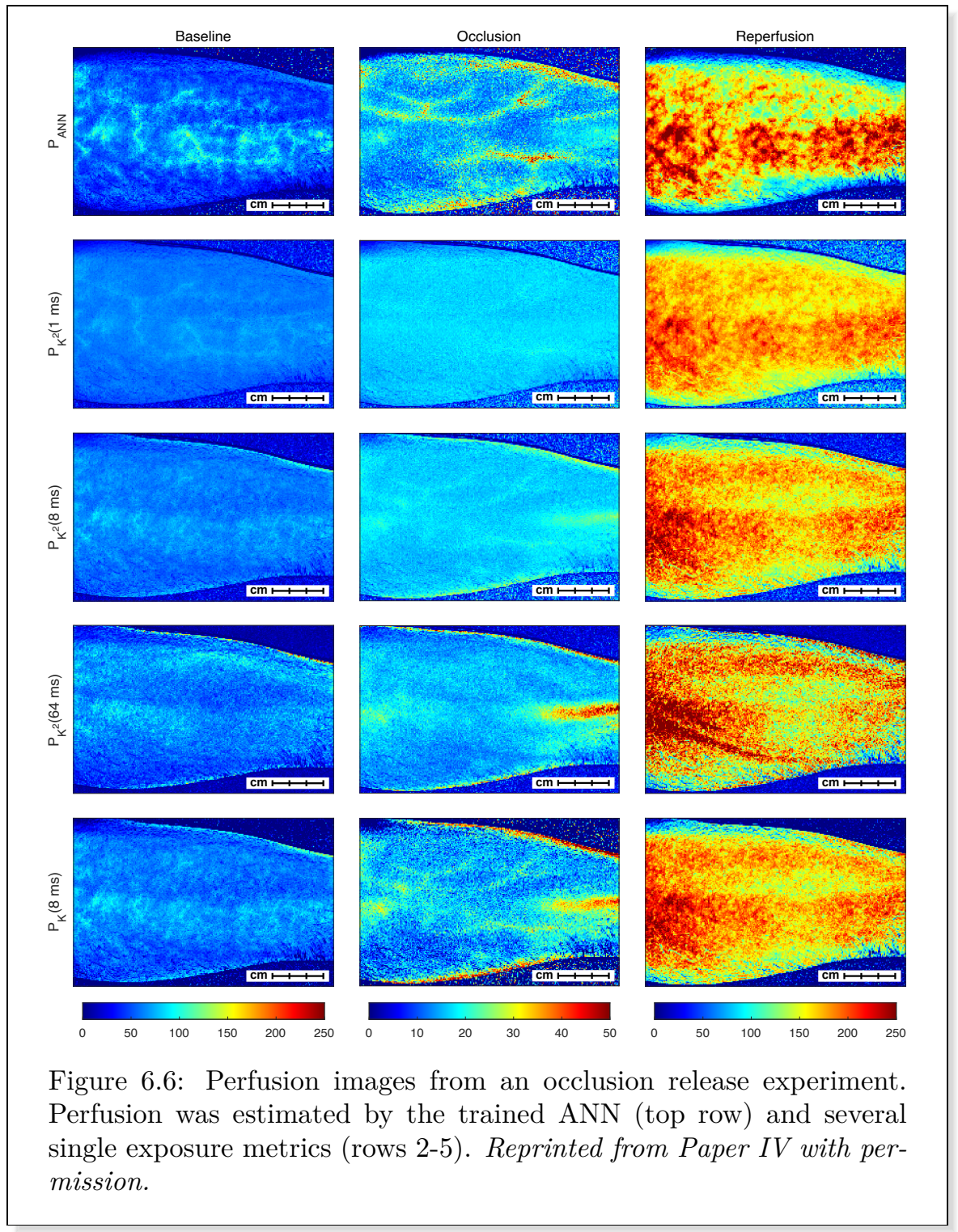




\subsection{Paper V}

The fifth paper, titled Comprehensive imaging of microcirculatory changes in the foot during endovascular intervention - a technical feasibility study, described the use of the MELSCI system for real-time monitoring of the microcirculation in subjects with chronic limb-threatening ischemia (CLTI). We used the MELSCI system, together with a complementary multi-spectral imaging (MSI) system for oxygen saturation, to monitor the spatiotemporal microcirculatory changes in the foot sole while the subjects were treated by percutaneous balloon angioplasty and stent placements. The aim was to investigate the technical feasibility of the multimodal instrument in-vivo, and to present potential value of both spatial and temporal information about the microcirculation to support peri-operative decisions.

First, we demonstrated that expected changes in the microcirculation could be observed in the data following intra-arterial occlusion by balloon dilation, where both the perfusion and oxygen saturation decreased and subsequently increased after the deflation. Similarly, following administration of a vasodilator, we observed a substantial increase in both measurements followed by a slow decrease several minutes later. This event is presented in Figure 6.7. The purpose of these cases was to mimic the potential use as a decision making tool, where perfusion and oxygen saturation could be compared in real-time before and after each step of the intervention. The data exhibited low spatial and temporal noise, despite the difficult measurement setting. For example, it was possible to extract pulsatility information in addition to long-term changes.

In addition to the expected changes, we also observed unexpected dynamics in both the perfusion and oxygen saturation, both spatially and temporally. In one subject, we observed a rapid occlusion locally in two toes, while the rest of the data was unchanged, as seen in Figure 6.8. We hypothesized that this could be caused by a micro-emboli, which was further supported by reports of pain from the patient. While this particular occlusion dissolved spontaneously after about an hour, detecting larger emboli could be a valuable tool for interventionists. Another unexpected dynamic was observed in two subjects, where especially the oxygen saturation images showed a very heterogeneous random pattern. The cause of this was unknown.

One major issue with the technique, at the time of writing, was the amount of manual labor and expert knowledge required to extract and distil the results. Of course this is nothing unusual with new technology, but it was nevertheless clear that future studies should aim to develop automatic algorithms that can detect, follow, and compare changes in the collected data. For example, an analysis based on thresholds and derivatives in perfusion and oxygen saturation might detect when an intervention action propagates to the microcirculation, or provide warnings of adverse changes such as the suspected embolization in this work. An analysis of the spatial heterogene- 

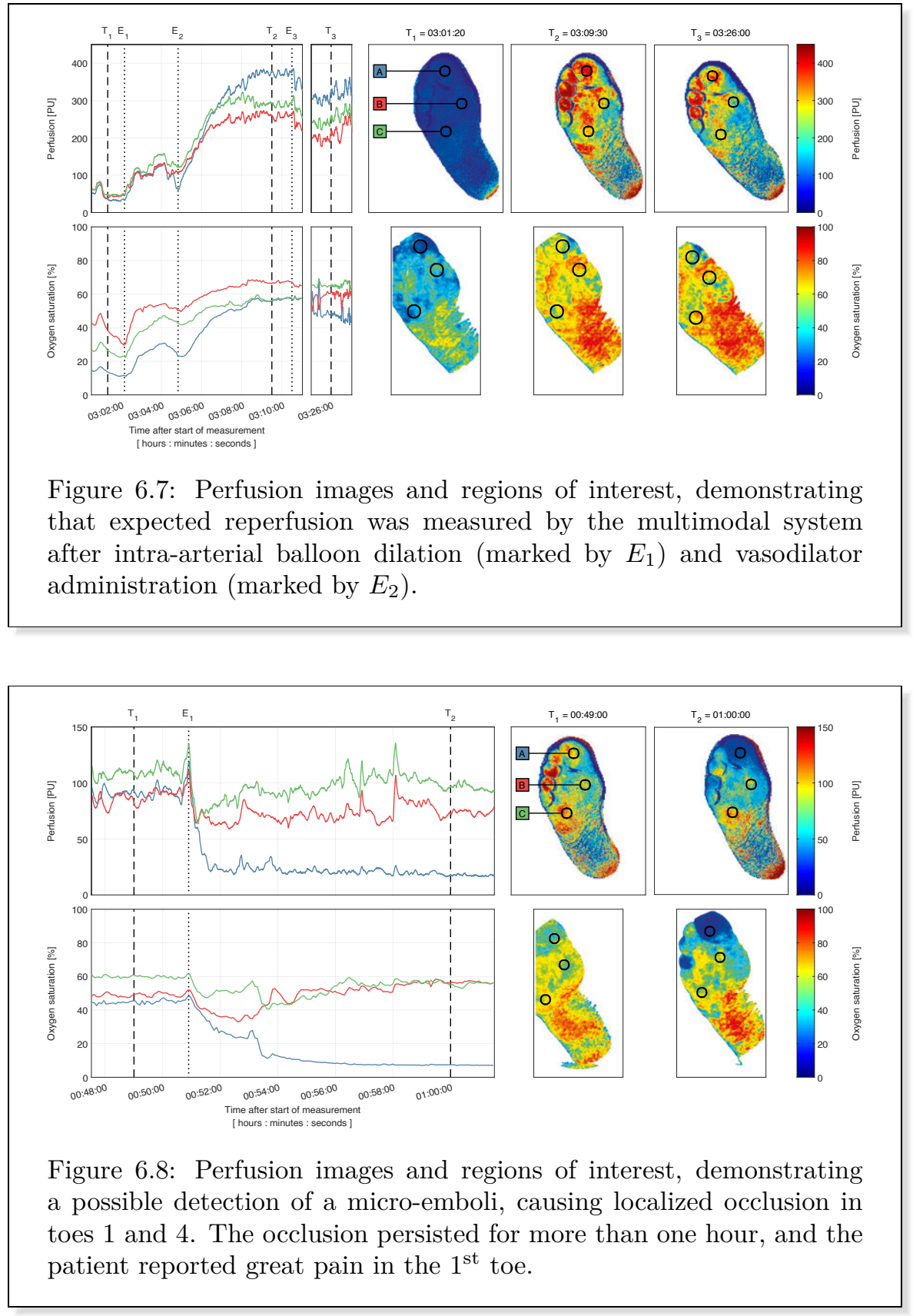
ity in the data might provide similar information. Regardless, it was clear that a substantial engineering effort is required before the technique can be adopted in daily practice. It should also be noted that the intent of this study was not to show the clinical value of the results, which would require a significantly larger sample size, but only to investigate the feasibility of the technique in this setting. The clinical value should be investigated in future studies, preferably in pre-, peri-, and post-operative measurements.

\subsection{Paper VI}

In the final paper of this thesis, titled Speed-resolved perfusion imaging using multi-exposure laser speckle contrast imaging and machine learning, we proposed a model for estimating speed-resolved skin perfusion in absolute units $\% \mathrm{RBC} \times \mathrm{mm} / \mathrm{s}$ from MELSCI data. As described in Chapter 4, the true perfusion in the skin tissue models can be computed in different speed ranges. Here, we computed the three speed ranges $0-1 \mathrm{~mm} / \mathrm{s}, 1-10 \mathrm{~mm} / \mathrm{s}$, and $>10 \mathrm{~mm} / \mathrm{s}$, as well as the total perfusion given by their sum.

Like in Paper III, we trained an artificial neural network on a dataset of 100000 simulated measurements, with multi-exposure contrast as input, and the three speed components as output. The trained ANN was first evaluated on simulated data, and presented estimation errors of $9.5 \%, 10.3 \%, 15.9 \%$, and $29.5 \%$, for the three speed components and total perfusion, respectively. Results also showed that, compared to LSCI perfusion (Equation 3.19), the ANN perfusion was more linear with respect to changes in RBC speeds, and had a near to linear response to changes in $\mathrm{RBC}$ concentration relevant for skin.

We also investigated how changes in tissue parameters - scattering coefficient, average vessel diameter, epidermis thickness, epidermis absorption, and oxygen saturation - affected the estimated perfusion. The estimation error of the ANN was lower than that of LSCI perfusion, for all parameters, and all tested parameter values. For the latter two parameters, the error for both the ANN perfusion and LSCI perfusion was less than $2 \%$ over a wide range of values, as expected. Changes in the former three parameters caused larger errors in the perfusion estimates at extreme values of the parameters, especially for low scattering and low average vessel diameters. These error sources are important when considering inter-subject comparisons. However, when measuring in a single individual, many confounding factors are static, resulting in a linear response to changes in true perfusion.

We evaluated the speed-resolved perfusion images in an arterial occlusion of the forearm of a healthy volunteer, as shown in Figure 6.9. Two observations were made. First, the perfusion in the $0-1 \mathrm{~mm} / \mathrm{s}$ speed component was more homogeneous than in the $>10 \mathrm{~mm} / \mathrm{s}$ speed component, where the perfusion was more concentrated to specific high-perfusion spots in the image. This 
is physiologically reasonable, since the larger vessel structures deeper in the tissue should have higher average flow speeds, and thus the $>10 \mathrm{~mm} / \mathrm{s}$ speed component should be more sensitive to these vessels. Conversely, in general, the capillary flow is lower and thus the $0-1 \mathrm{~mm} / \mathrm{s}$ speed component should present a more homogeneous perfusion. The second observation was that the perfusion estimated during occlusion was significantly less noisy than in LSCI, sufficiently so that the large veins could clearly be seen in the images. That perfusion is measured in the large vessels during occlusion is physiologically reasonable, since the RBCs in these vessels experience Brownian motion, as well as a redistribution of blood from the arterial to venous side [94].

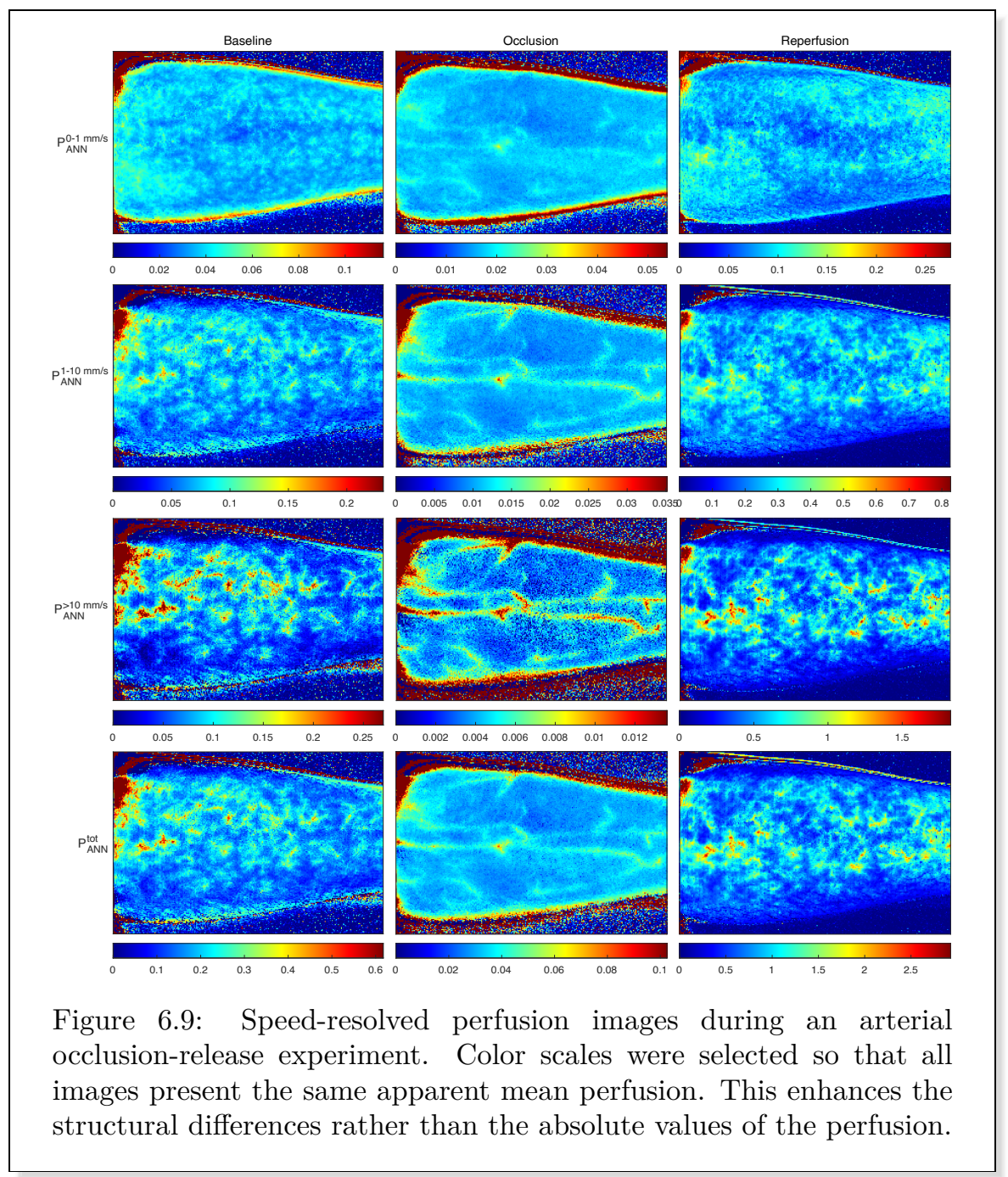


We also presented speed-resolved perfusion in two regions of interest (ROIs), selected on a low-perfusion and high-perfusion spot on the tissue, respectively. This is shown in Figure 6.10. Both absolute and relative changes in perfusion supported the hypothesis that the speed components are indeed sensitive to different parts of the microvascular network, although more research is required to confirm this. For example, the $>10 \mathrm{~mm} / \mathrm{s}$ speed component decreased more during occlusion than the lower speed components. This can be explained by a shift in the speed-distribution towards low-speed Brownian motion and redistribution flow, as mentioned above. The opposite was observed in the reperfusion peak, where the $>10 \mathrm{~mm} / \mathrm{s}$ speed component had a significantly larger relative increase, compared to baseline, than the $0-1 \mathrm{~mm} / \mathrm{s}$ speed component.

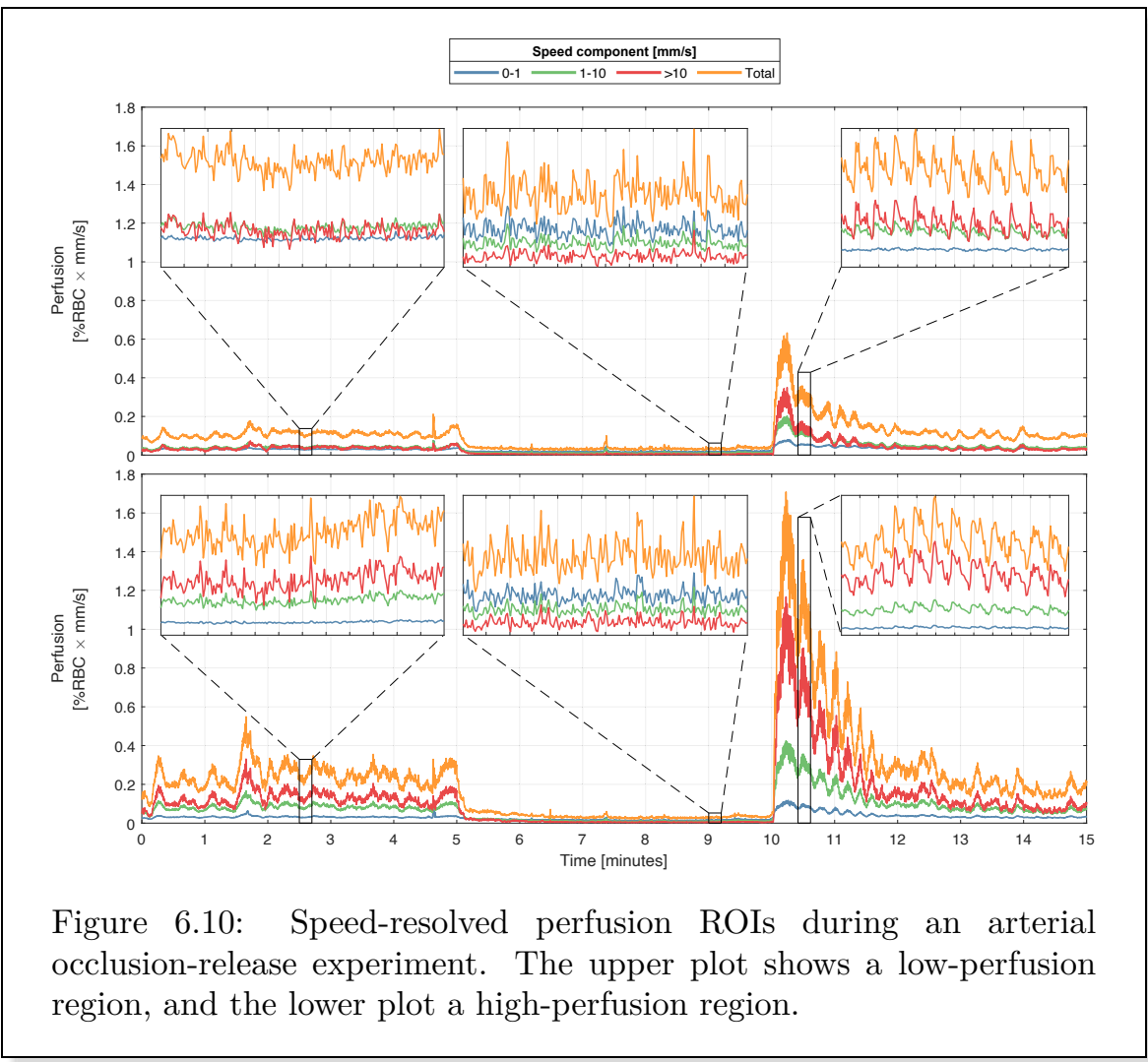




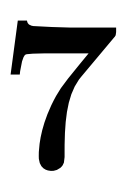

\section{Discussion}

This thesis has proposed solutions to several shortcomings of MELSCI, by mathematical modeling of speckle contrast and perfusion, by real-time contrast algorithms with optimal use of available data, and by the development of an integrated system that enables the use of these results in clinical applications. This chapter presents a discussion of these results.

\subsection{Clinical potential}

The high clinical potential of MELSCI compared to LSCI is clear. On a basic level, the potential accuracy and reliability of MELSCI is fundamentally higher than LSCI, due to the higher information content in multiple exposures. For example, as seen in Figure 3.7, it is possible to measure the same contrast with LSCI from significantly different tissue states. Furthermore, as reported by Liu et al. [95], the necessary assumptions in LSCI can lead to estimation errors as high as 300\%, which is not the case in MELSCI. Despite this high potential, there have been a lack of ideas beyond the inclusion of the Doppler fraction $\rho$ to account for static scattering. No efforts have been made to reexamine the use of $\tau_{c}$ and instead base the models on physiological parameters.

This thesis addresses this by presenting two ANN models for estimating skin perfusion from multi-exposure contrast, one targeting conventional LDF perfusion [Paper III], and one targeting speed-resolved true perfusion [Paper VI]. Both avoid the assumptions of conventional LSCI and previous MELSCI models, and therefore provide an immediate increase in interpretability and, as the results of Paper III shows, accuracy. While the estimation accuracy of quantitative speed-resolved true perfusion could potentially be increased by use of multi-modal measurements, the quality of the speed-resolved perfusion images presented in Paper VI is sufficient to warrant further use of the proposed ANN. If the confounding factors in the estimate of true perfusion can be addressed, this could potentially lead to more accurate comparisons between individuals, as well as on a population level, greatly increasing the utility of the technique. For example, this could enable threshold-based decision making in new clinical applications, such as for diagnosis of PAD and CLTI, similar to how ABI and TBI are used today. Currently, measurements with LSCI 
or MELSCI can often only be interpreted in combination with provocations, e.g. thermal stimuli, elevation, or cuff occlusion. These are time-consuming for the clinicians, and sometimes painful for the patient. Quantitative perfusion could solve this, by enabling more accurate direct comparison of baseline measurements.

LSCI has been a valuable research tool due to the high spatio-temporal resolution. Although it was demonstrated that conventional MELSCI perfusion (Equation 3.21) was superior in terms of linearity to true perfusion [19], LSCI is still the more used technique. High spatio-temporal resolution is important, since the microcirculation is both a spatially heterogeneous and temporally dynamic system. For example, in diagnosis and treatment of CLTI, the pulsatility has been used as an important marker of tissue viability [50]. The same potential has been demonstrated in the assessment of burns [44]. High temporal resolution also reduces the impact of motion artifacts, which is especially important in applications like in Paper V, where long-term monitoring is required and preventing motion artifacts is difficult. The instrument presented in this thesis is the first time high spatio-temporal resolution has been combined with the benefits of a more accurate perfusion model. As such, we should expect that the new technique could be useful in any application where LSCI has shown promise, with more accurate and reliable results. There are also a few areas in which the benefits could extend beyond this, especially with the addition of speed-resolved perfusion.

In general, speed-resolved perfusion has potential in applications where there are processes to separate in different types of vessels. For example, the capillary degradation in diabetes has been investigated with speed-resolved perfusion computed from the Doppler power spectrum in $\operatorname{LDF}[96,97,98]$. However, the spatial heterogeneity in perfusion might make comparisons difficult. This is less of an issue in speed-resolved perfusion imaging, where the analysis could be performed in multiple tissue regions with low or high perfusion, as in Paper VI, or as an average over a large area. Another application where different vessels are affected differently is in burns. As presented in the introduction, LSCI has been used in a few studies to estimate the healing potential of burns. The healing potential is related to burn area, as well as the depth of the wound and the corresponding degree of damage to different layers of the skin [99]. It is therefore reasonable to assume that speed-resolved perfusion, due to the selective sensitivity to different flow speeds, could provide valuable additional information about the degree of injury. Combined with models for automatic prediction of healing potential, for example using deep learning for classification [100] or semantic segmentation [101, 102], this has the potential to aid the decision making regarding the need for surgery.

The frequency content in the perfusion signal is also of interest. For LDF, analysis using the wavelet spectrum of the perfusion signal was proposed by Stefanovska et al. [103], and later used by several groups [55]. This relies on estimating the energy in distinct frequency bands related to different pro- 
cesses in the microcirculation; cardiac, respiratory, myogenic, neurogenic, and endothelial activity $[104,105]$. For example, it has been shown that these energy levels are different in diabetic subjects compared to healthy controls [106]. This analysis relies on a high sample rate, to resolve the fast dynamics of the heart-related variations, but also a long measurement time, to resolve the lowfrequency dynamics related to myogenic, neurogenic, and endothelial activity [104]. The methods and instrument presented in this thesis potentially enable the same analysis in an imaging modality, allowing simultaneous access to any location on the tissue, such as high-perfusion and low-perfusion areas. The separation into speed-resolved perfusion might also enable new tests, by performing the frequency analysis on the individual speed components, loosely representing different vasculatures. This was recently demonstrated in speedresolved LDF [107].

\subsection{Strengths and limitations of the FPGA-based implementation}

\section{Possible implementation in GPU}

The FPGA-based solution presented in this thesis creates a steep engineering cost to recreate the results. Early on, we decided to take this route instead of, for example, an implementation in GPU. The required data throughput limited the number of solutions to very high-performance hardware. While an implementation of the MELSCI algorithms in GPU could potentially have been even faster than the final FPGA implementation, this required the data to be located in the GPU memory. We did not see an obvious solution for streaming the camera data directly into the graphics memory, meaning the only solution would have been to move the data through the CPU and RAM, for example using a frame-grabber PCIe card which connected the camera. We evaluated this method in early testing but discovered a bottleneck in the memory bandwidth, making real-time applications infeasible. For this reason, we did not attempt to build a GPU solution. Instead, we opted for the presented solution where data was processed and compressed on the FPGA before transfer to the computer, circumventing the restrictions we found with the frame-grabber. That said, computer hardware such as GPUs and memory technology have advanced significantly since the start of this project, and it is quite possible that there are now solutions which would not require the FPGA. If an easy solution based on a frame-grabber (or equivalent) and GPU could be shown, then the results from this project would become more accessible to other research groups.

However, even if a GPU-based solution could be developed, there are merits to the integrated FPGA-solution. For example, requiring a GPU is restrictive, since this also requires the computer to utilize it. While this is not a problem in a research setting, when considering implementation in routine 
clinical use, such a requirement is more disruptive. The FPGA-based solution, on the other hand, is a very versatile self-contained solution which can be used with any lightweight portable computer. In principle, it could even be extended so that the device could be connected directly to a display, or even stream the final images to a smart device over Bluetooth. Whether or not this would be useful remains to be seen, but the point is that the platform was designed to allow easier deployment in real use, not just the research lab.

\section{Number of speckles per pixel}

One current limitations of this system is the high number of speckles per pixel. It is often stated in speckle imaging that the speckle size should be equal to or larger than the pixel size, i.e. the ratio of pixels to speckles should satisfy $n_{p} / n_{s} \geq 1$. It has been shown that this ratio should exceed the Nyqvist criterion $n_{p} / n_{s} \geq 2$ in order to obtain the theoretical maximum contrast of $K=1$ on a static object [68]. Due to the short exposure time in our system (1 ms), a large aperture is required to capture sufficient light, which is important to avoid quantization errors in the contrast calculations [108]. We currently use an f-number $f_{\#}=1.4$, causing small speckles $\left(n_{p} / n_{s}<<1\right)$ and thus a decrease in contrast through speckle averaging. Calibrating the system using $\beta=1 / K_{\max }^{2}$ as described in Chapter 3 is a valid solution. This has been demonstrated both theoretically [63], and in practice [109, 110, Paper III]. However, when the maximum contrast becomes too small, quantization noise in the calculations and structure-related (non-speckle) contrast from the background might become too large to benefit from the increased light level gained from the large aperture. The exact ratio $n_{p} / n_{s}$ where this becomes a problem has not been investigated.

\section{Size of the contrast kernel}

We implemented the contrast calculations using a $4 \times 4$ kernel. This choice was made to enable a more efficient implementation in the FPGA. For example, division by 16 , which is necessary to compute the variance, can be done in binary by right-shifting the value by 4 bits, since 16 is a power of 2 . This operation is so fast that it can even be implemented in the combinatorial logic as part of other steps in the variance calculation, making it essentially instant. Contrary to this, if a division by a non power of 2 is required, say 25 , this is a much slower process in comparison. The next power of 2 kernel size is $8 \times 8$, which would reduce the size of the contrast images to $128 \times 160$ pixels, too small to resolve interesting details in the perfusion images over a large area.

Of course, this argument is based on the fact that we do not implement overlapping contrast kernels. However, there is no fundamental reason why such a solution could not be implemented. Overlapping the $4 \times 4$ kernels 
by 2 pixels would increase the size of the contrast images to $511 \times 639$ pixel (loosing one pixel at the top and left edges). This would also increase the processing time by 4 , but this would still be within the real-time constraints. Preferably, the kernel size should be configurable by the user, but the current FPGA implementation trades flexibility for efficiency, making such functionality difficult. More efficient algorithms for processing speckle images have been proposed [111]. Especially interesting is the "roll" algorithm presented there, since it removes redundant arithmetic operations, and enables a kernel of any size $m \times n$. The issue with division by non powers of 2 remains, however. One possibility would be to rewrite the equations so that only a single division has to be performed. This would instead introduce multiplications, but these are generally fast if implemented using the dedicated primitives in the FPGA.

\subsection{Interpretability of artificial neural networks}

One of the main drawbacks of artificial neural networks, compared to the analytical models in Chapter 3, is that they are black-box models. In other words, it is exceedingly difficult to understand their inner workings. Some machine learning models are more transparent, such as decision trees or support vector machines, but ANNs in particular are very opaque. A reasonable question is whether the better accuracy, less noise, and fewer assumptions are worth the cost of not understanding why the models work. There are a few factors that make this issue less problematic in our case.

The presented ANNs are trained on simulated data built by a framework of well defined mathematical models, which gives us much greater tools to investigate their behaviour. One direct example of this is in Paper VI, where we investigated how different skin parameters influenced the ANN prediction error. This experiment was easy to perform due to the simulated model, where we had full control over the parameters, and we could generate unlimited data. Performing this experiment with real tissue-like phantoms would be difficult, if at all possible.

Furthermore, one reason that many trained machine learning model are difficult to interpret, is that there are no other solution to the problems they solve. This is not the case with our computational model. During the development process we have also applied a more traditional iterative inverse solver to fit the computational model to the simulated contrast data, with reasonable success. This method is very transparent, and thus provides a mean to indirectly investigate the perfusion ANNs. For example, if the ANN has a high estimation error in a skin model, the inverse solver can be used to investigate if this is a fault of the $\mathrm{ANN}$, or a case where the information about the perfusion is not contained in the contrast. The problem with inverse solvers is that they are slow, like we show in Paper II and Paper III for 
an analytical MELSCI perfusion model (Equation 3.21), and thus cannot be used in real-time applications. The ANNs solve this by moving the slow part of the algorithm to the training phase of the network, while the inference time is very fast. Essentially, the ANNs (or comparable learned models) are necessary because they make the perfusion imaging technique practically useful, not because they solve an otherwise unsolvable problem.

There are also techniques to investigate the inner workings of neural networks, such as gradient ascent on the input to maximize specific activations, or by statistical analysis of the activations given the input samples. While these techniques have, to my best knowledge, mostly been used to analyze deep convolutions neural networks (CNNs) for image analysis, they are just as applicable to our 1-dimensional data. In fact, the small size of our networks might allow insight that typically is not possible when using neural networks. In deep learning models, the number of parameters are often in the millions [112], such as AlexNet (61M) [113] and ResNet (25.5M) [114], or the more recent GPT-3 with a staggering 175 billion parameters [115]. Our models from Paper III and Paper VI have just 91 and 278 parameters, respectively. This might enable direct analysis of the purpose of each weight or node in the network, something that would be impossible in models with millions of learned parameters. It should however be noted that there are criticisms towards this type of analysis, instead of using models that are inherently interpretable [116].

\subsection{Assumptions in conventional contrast models}

In contrast to ANNs, the analytical models presented in Chapter 3 are inherently interpretable through the optimal parameters. For example as in Equation 3.21, where the multi-exposure contrast is modeled by $\tau_{c}, \rho$, and $\beta$, which all have direct interpretations: $\tau_{c}$ - the time in which $g^{(1)}(\tau)$ decreases to $37 \%$ of it's maximum, $\rho$ - the fraction of Doppler shifted light, and $\beta$-inverse of the squared contrast measured on a static object. But the interpretation is only useful if the model is a sufficiently complete description of reality, and if the parameters represent the properties of interest. The conventional contrast models are based on three assumptions in particular that are questionable in measurements of skin microcirculation, and likely in other tissues as well:

1. "The field autocorrelation function $g^{(1)}(\tau)$ can be modeled as a Gaussian [71], Lorentzian [71], or Voigt [72] distribution." This might be valid in cases where large visible surface vessels are analyzed, for example when imaging the brain, and this is where MELSCI has been almost exclusively used prior to this thesis $[21,30,39,40,117,118,119]$. However, this assumption is too restrictive when considering the skin microcircu- 
lation, where the influence on $g^{(1)}(\tau)$ from the tissue properties is more complex.

2. "There is a simple inverse connection between speckle decorrelation time $\tau_{c}$ and RBC speed" [16] (see Equation 3.17). This assumption is likely false in all tissues, including the brain. Duncan et al. [72] wrote, in regards to the original publication: "The authors admit that this relationship is speculative and give no first principles argument as to its veracity. Nevertheless this relationship has entered the literature as fact.". Even the original authors admit the same: "The relationship between $\tau_{c}$ and the mean (or some typical) velocity is also open to speculation. The simplest approach leads to a characteristic decorrelation velocity [...]" [16].

3. "No photons are Doppler-shifted more than once." [39] The inaccuracy of this assumption has been known since the first articles on $\operatorname{LDF}[1,3]$, and has been demonstrated using Monte Carlo simulations [81].

As mentioned previously, Liu et al. [95] recently investigated these and other assumptions, and found that in cases where multiple wrong assumptions coincided, errors as high as $300 \%$ could be observed for LSCI.

The computational model presented in this thesis relies on none of these assumptions. There are other assumptions in the presented model, but those are made on the physiological parameters (for example the speed distribution of RBCs or the distribution of tissue parameters), and can easily be adjusted to target specific applications not covered by the current parameter space.

\subsection{The unknowable ground truth}

A problem with all perfusion models for skin microcirculation is that they are essentially unverifiable, due to the complex structure and function of the microcirculation. Since there is no accepted gold-standard that can measure the skin perfusion in real-time to capture the temporal dynamics, it is impossible to truly know if the perfusion models are accurate. For other tissue morphologies, such as on the surface of the brain or the retina, individual vessels are visible and models can be validated through tube-phantoms where the flow is a controllable experimental parameter, for example as in Ref [120]. But a tube-phantom that accurately mimics the structure of the skin microcirculation, especially as seen in wide-field imaging, would be so complex that the manufacturing process would be unfeasible, and it would be difficult to know the true flow in the phantom, defeating the purpose.

For the specific perfusion models developed in this thesis, i.e. the ANNs from Paper III and Paper VI, the validation on a tube phantom would most likely not yield satisfactory results, since that tissue morphology is not part of the current training space. Of course, it would be possible to change the 
training data to target this situation, at which point the tube phantoms would be a good method of validation. However, this would not validate the original ANNs trained for skin perfusion, only the new ANNs trained for the phantom measurements. The most we could conclude from such an experiment would be the validity of the data generation and network training methodology, but not the validity of the specific ANNs presented in this thesis.

There might be other methods that can be useful for validation. Early work on LDF by Braverman and Schechner [6] used histological evaluation, although this is not a desirable method due to the invasiveness. It would also be very difficult to connect the histology to the perfusion measurements. A non-invasive alternative might be to use optical coherence tomography angiography (OCTA) to map the microvascular network in a small tissue volume, recently demonstrated in human skin [121], and correlate this to perfusion images of the same tissue.

\subsection{Future work}

This thesis presents the first imaging instrument capable of estimating speedresolved perfusion. As such, the clinical value of the technique has not been demonstrated. Although the potential is clear, as discussed above, the instrument must now be validated in both laboratory experiments and in clinical trials. Previous studies on speed-resolved perfusion have shown potential benefits from the ability to differentiate different vessel types, and this is likely also where the speed-resolved perfusion images would be most beneficial.

The results in Paper VI, regarding the influence of several tissue parameters on the estimated perfusion, clearly shows that MELSCI is not a sufficient technique on its own to obtain a truly quantitative perfusion estimate with low absolute error. The scattering and vessel diameter have especially large impact on the perfusion accuracy. The only possibility to correct for these effects is to measure them, which requires complementary methods such as spatial frequency domain imaging (SFDI) and multi-spectral imaging (MSI). Systems using SFDI fully integrated with LSCI [122] or MELSCI [123] have been demonstrated, i.e. using a spatially modulated coherent light source. These were demonstrated in multilayered phantoms, although the approach might not be feasible for real-time measurements as presented in this thesis. We presented a multi-modal instrument with MELSCI and MSI in Paper V, but the measurements were only processed separately, and not in a combined model that could benefit from the information of the two techniques. This would require a near pixel-to-pixel mapping between the two images, which could prove to be a significant engineering challenge. If we also require SFDI, this becomes even more difficult to integrate. Regardless, this is likely the best approach for the future, not only to correct for the effects on perfusion, 
but also to enable the estimation of new physiologically relevant quantities such as the tissue metabolic rate of oxygen [124].

\subsection{Concluding remarks}

The purpose of this thesis was to address several shortcomings of conventional LSCI and MELSCI, and was divided into four aims. These have all been achieved:

1. In Paper I we presented a proof-of-concept implementation of synthetic MELSCI in real-time. In Paper II, we evaluated this system with a previous analytic model for MELSCI-based perfusion. The limitations discovered with the system and model were addressed in Paper IV, where a 15.6 fps MELSCI system was demonstrated without loss of data.

2. In Paper III, we proposed a method to compute multi-exposure contrast and perfusion from simulated skin models, for training machine learning models. This included proposing a model for adding realistic speckle realization noise to the contrast, to achieve generalization from simulations to in-vivo data. We demonstrated the fast computational speed of an ANN trained on this data, enabling real-time perfusion imaging at the full $15.6 \mathrm{fps}$.

3. In Paper VI, we used the methodology from Paper III to train an ANN for real-time estimation of speed-resolved perfusion in absolute units, and demonstrated the linear response to both RBC speeds and RBC concentrations, as well as the lower susceptibility to tissue parameters compared to LSCI.

4. In Paper V, we used the instrument for peri-operative imaging of foot perfusion during endovascular surgery for critical limb-threatening ischemia, demonstrating the potential future use as a decision support tool. High quality images were obtained despite the difficult measurement setting, due to the fast framerate and low susceptibility to noise in the perfusion estimate.

In conclusion, the advancements to MELSCI proposed in this thesis has the potential to improve the clinical viability of the technique, increase interpretability of the results, and might lead to improved treatments based on a better understanding of the complex processes in the microcirculation. 


\section{Bibliography}

[1] M. D. STERN. "In vivo evaluation of microcirculation by coherent light scattering." In: Nature 254.5495 (Mar. 1975), pp. 56-58. ISSN: 1476-4687. DOI: 10.1038/ $254056 \mathrm{a} 0$.

[2] M. D. Stern, D. L. Lappe, P. D. Bowen, J. E. Chimosky, G. A. Holloway, H. R. Keiser, and R. L. Bowman. "Continuous measurement of tissue blood flow by laserDoppler spectroscopy." In: American Journal of Physiology-Heart and Circulatory Physiology 232.4 (1977), H441-H448. DOI: 10.1152/ajpheart.1977.232.4.H441.

[3] R. Bonner and R. Nossal. "Model for laser Doppler measurements of blood flow in tissue." In: Appl. Opt. 20.12 (June 1981), pp. 2097-2107. DOI: 10.1364/A0.20. 002097.

[4] G. E. Nilsson. "Signal processor for laser Doppler tissue flowmeters." In: Medical and Biological Engineering and Computing 22.4 (July 1984), pp. 343-348. ISSN: 1741-0444. DOI: $10.1007 / \mathrm{BF} 02442104$.

[5] T Tenland, E G Salerud, G E Nilsson, and P A Oberg. "Spatial and temporal variations in human skin blood flow." In: Int J Microcirc Clin Exp 2.2 (1983), pp. 81-90.

[6] Irwin M Braverman and Jeffrey S Schechner. "Contour Mapping of the Cutaneous Microvasculature by Computerized Laser Doppler Velocimetry." In: Journal of Investigative Dermatology 97.6 (1991), pp. 1013-1018. ISSN: 0022-202X. DOI: 10.1111/1523-1747.ep12492255.

[7] Irwin M. Braverman, Jeffrey S. Schechner, David G. Silverman, and Agnes KehYen. "Topographic mapping of the cutaneous microcirculation using two outputs of laser-Doppler flowmetry: Flux and the concentration of moving blood cells." In: Microvascular Research 44.1 (1992), pp. 33-48. ISSN: 0026-2862. DOI: $10.1016 /$ 0026-2862 (92) 90100-4.

[8] G.E. Nilsson, A. Jakobsson, and K. Wardell. "Imaging of tissue blood flow by coherent light scattering." In: Images of the Twenty-First Century. Proceedings of the Annual International Engineering in Medicine and Biology Society, 1989, 391392 vol.2. DOI: 10.1109/IEMBS.1989.95785.

[9] K. Wårdell, A. Jakobsson, and G.E. Nilsson. "Laser Doppler perfusion imaging by dynamic light scattering." In: IEEE Transactions on Biomedical Engineering 40.4 (1993), pp. 309-316. DOI: 10.1109/10.222322.

[10] Alexander Serov, Wiendelt Steenbergen, and Frits de Mul. "Laser Doppler perfusion imaging with a complimentary metal oxide semiconductor image sensor." In: Opt. Lett. 27.5 (Mar. 2002), pp. 300-302. DOI: 10.1364/OL.27.000300.

[11] Alexandre Serov, Beda Steinacher, and Theo Lasser. "Full-field laser Doppler perfusion imaging and monitoring with an intelligent CMOS camera." In: Opt. Express 13.10 (May 2005), pp. 3681-3689. DOI: 10.1364/OPEX.13.003681.

[12] Alexandre Serov and Theo Lasser. "High-speed laser Doppler perfusion imaging using an integrating CMOS image sensor." In: Opt. Express 13.17 (Aug. 2005), pp. 6416-6428. DOI: $10.1364 /$ OPEX.13.006416. 
[13] Marcel Leutenegger, Erica Martin-Williams, Pascal Harbi, Tyler Thacher, Wassim Raffoul, Marc André, Antonio Lopez, Philippe Lasser, and Theo Lasser. "Realtime full field laser Doppler imaging." In: Biomed. Opt. Express 2.6 (June 2011), pp. 1470-1477. DOI: 10.1364/BOE.2.001470.

[14] A.F. Fercher and J.D. Briers. "Flow visualization by means of single-exposure speckle photography." In: Optics Communications 37.5 (1981), pp. 326-330. ISSN: 0030-4018. DOI: 10.1016/0030-4018(81)90428-4.

[15] J.D. Briers and S. Webster. "Quasi real-time digital version of single-exposure speckle photography for full-field monitoring of velocity or flow fields." In: $O p$ tics Communications 116.1 (1995), pp. 36-42. ISSN: 0030-4018. DOI: 10.1016/00304018(95) 00042-7.

[16] J. David Briers and Sian Webster. "Laser speckle contrast analysis (LASCA): a nonscanning, full-field technique for monitoring capillary blood flow." In: Journal of Biomedical Optics 1.2 (1996), pp. 174-179. DOI: 10.1117/12.231359.

[17] J David Briers. "Laser Doppler, speckle and related techniques for blood perfusion mapping and imaging." In: 22.4 (Oct. 2001), R35-R66. DoI: 10.1088/0967-3334/ $22 / 4 / 201$.

[18] Andrew K. Dunn, Hayrunnisa Bolay, Michael A. Moskowitz, and David A. Boas. "Dynamic Imaging of Cerebral Blood Flow Using Laser Speckle." In: Journal of Cerebral Blood Flow \& Metabolism 21.3 (2001), pp. 195-201. DOI: 10 . 1097 / 00004647-200103000-00002.

[19] Ashwin B. Parthasarathy, W. James Tom, Ashwini Gopal, Xiaojing Zhang, and Andrew K. Dunn. "Robust flow measurement with multi-exposure speckle imaging." In: Opt. Express 16.3 (Feb. 2008), pp. 1975-1989. DOI: 10.1364/0E.16.001975.

[20] Oliver B. Thompson and Michael K. Andrews. "Tissue perfusion measurements: multiple-exposure laser speckle analysis generates laser Doppler-like spectra." In: Journal of Biomedical Optics 15.2 (2010), pp. 1-7. DOI: 10.1117/1.3400721.

[21] Tanja Dragojević, Danilo Bronzi, Hari M. Varma, Claudia P. Valdes, Clara Castellvi, Federica Villa, Alberto Tosi, Carles Justicia, Franco Zappa, and Turgut Durduran. "High-speed multi-exposure laser speckle contrast imaging with a single-photon counting camera." In: Biomed. Opt. Express 6.8 (Aug. 2015), pp. 2865-2876. DOI: 10.1364/BOE.6.002865.

[22] Shen Sun, Barrie R. Hayes-Gill, Diwei He, Yiqun Zhu, and Stephen P. Morgan. "Multi-exposure laser speckle contrast imaging using a high frame rate CMOS sensor with a field programmable gate array." In: Opt. Lett. 40.20 (Oct. 2015), pp. 4587-4590. DOI: 10.1364/OL.40.004587.

[23] "Comparison of laser Doppler and laser speckle contrast imaging using a concurrent processing system." In: Optics and Lasers in Engineering 83 (2016), pp. 1-9. ISSN: 0143-8166. DOI: 10.1016/j.optlaseng.2016.02.021.

[24] J D Briers and A F Fercher. "Retinal blood-flow visualization by means of laser speckle photography." In: Investigative Ophthalmology \& Visual Science 22.2 (Feb. 1982), pp. 255-259. ISSN: 1552-5783.

[25] Wido Heeman, Wiendelt Steenbergen, Gooitzen M. van Dam, and E. Christiaan Boerma. "Clinical applications of laser speckle contrast imaging: a review." In: Journal of Biomedical Optics 24.8 (2019), pp. 1-11. DOI: 10.1117/1. JBO.24.8. 080901.

[26] Tetsuya Sugiyama, Makoto Araie, Charles E. Riva, Leopold Schmetterer, and Selim Orgul. "Use of laser speckle flowgraphy in ocular blood flow research." In: Acta Ophthalmologica 88.7 (2010), pp. 723-729. DOI: $10.1111 / \mathrm{j} \cdot 1755-3768$. 2009 . 01586.x. 
[27] Miyuki Nagahara, Yasuhiro Tamaki, Atsuo Tomidokoro, and Makoto Araie. "In Vivo Measurement of Blood Velocity in Human Major Retinal Vessels Using the Laser Speckle Method." In: Investigative Ophthalmology $\&$ Visual Science 52.1 (Jan. 2011), pp. 87-92. ISSN: 1552-5783. DOI: 10.1167/iovs.09-4422.

[28] Xin Wei, Praveen Kumar Balne, Kenith E. Meissner, Veluchamy A. Barathi, Leopold Schmetterer, and Rupesh Agrawal. "Assessment of flow dynamics in retinal and choroidal microcirculation." In: Survey of Ophthalmology 63.5 (2018), pp. 646664. ISSN: 0039-6257. DOI: 10.1016/j.survophthal.2018.03.003.

[29] Anja Srienc, Zeb Kurth-Nelson, and Eric Newman. "Imaging Retinal Blood Flow with Laser Speckle Flowmetry." In: Frontiers in Neuroenergetics 2 (2010), p. 128. ISSN: 1662-6427. DOI: 10.3389/fnene.2010.00128.

[30] Lisa M Richards, SM Shams Kazmi, Katherine E Olin, James S Waldron, Jr Douglas J Fox, and Andrew K Dunn. "Intraoperative multi-exposure speckle imaging of cerebral blood flow." In: Journal of Cerebral Blood Flow \&6 Metabolism 37.9 (2017), pp. 3097-3109. DOI: 10.1177/0271678X16686987.

[31] Nils Hecht, Johannes Woitzik, Jens P. Dreier, and Peter Vajkoczy. "Intraoperative monitoring of cerebral blood flow by laser speckle contrast analysis." In: Neurosurgical Focus FOC 27.4 (2009), E11. DOI: 10.3171/2009.8.FOCUS09148.

[32] Nils Hecht, Johannes Woitzik, Susanne König, Peter Horn, and Peter Vajkoczy. "Laser speckle imaging allows real-time intraoperative blood flow assessment during neurosurgical procedures." In: Journal of cerebral blood flow and metabolism : official journal of the International Society of Cerebral Blood Flow and Metabolism 33.7 (July 2013), pp. 1000-1007. ISSN: 1559-7016. DOI: 10.1038/jcbfm.2013.42.

[33] Sadahiro Nomura, Takao Inoue, Hideyuki Ishihara, Hiroyasu Koizumi, Eiichi Suehiro, Fumiaki Oka, and Michiyasu Suzuki. "Reliability of Laser Speckle Flow Imaging for Intraoperative Monitoring of Cerebral Blood Flow During Cerebrovascular Surgery: Comparison with Cerebral Blood Flow Measurement by Single Photon Emission Computed Tomography." In: World Neurosurgery 82.6 (2014), e753-e757. ISSN: 1878-8750. DOI: $10.1016 / \mathrm{j}$. wneu.2013.09.012.

[34] Eva Klijn, Hester C. Hulscher, Rutger K. Balvers, Wim P. J. Holland, Jan Bakker, Arnaud J. P. E. Vincent, Clemens M. F. Dirven, and Can Ince. "Laser speckle imaging identification of increases in cortical microcirculatory blood flow induced by motor activity during awake craniotomy: Clinical article." In: Journal of Neurosurgery JNS 118.2 (2013), pp. 280-286. DOI: 10.3171/2012.10. JNS1219.

[35] Ashwin B. Parthasarathy, Erica L. Weber, Lisa M. Richards, Douglas J. Fox, and Andrew K. Dunn. "Laser speckle contrast imaging of cerebral blood flow in humans during neurosurgery: a pilot clinical study." In: Journal of Biomedical Optics 15.6 (2010), pp. 1-8. DOI: 10.1117/1.3526368.

[36] Lisa M. Richards, Erica L. Towle, Douglas J. Fox, and Andrew K. Dunn. "Intraoperative laser speckle contrast imaging with retrospective motion correction for quantitative assessment of cerebral blood flow." In: Neurophotonics 1.1 (2014), pp. 1-11. DOI: 10.1117/1.NPh.1.1.015006.

[37] Johannes Woitzik, Nils Hecht, Alexandra Pinczolits, Nora Sandow, Sebastian Major, Maren K.L. Winkler, Steffen Weber-Carstens, Christian Dohmen, Rudolf Graf, Anthony J. Strong, Jens P. Dreier, Peter Vajkoczy, and For the COSBID study group. "Propagation of cortical spreading depolarization in the human cortex after malignant stroke." In: Neurology 80.12 (2013), pp. 1095-1102. ISSN: 0028-3878. DOI: 10.1212/WNL. Ob013e3182886932. 
[38] Nils Hecht, Marc-Michael Müller, Nora Sandow, Alexandra Pinczolits, Peter Vajkoczy, and Johannes Woitzik. "Infarct prediction by intraoperative laser speckle imaging in patients with malignant hemispheric stroke." In: Journal of Cerebral Blood Flow \& Metabolism 36.6 (2016). PMID: 26661215, pp. 1022-1032. DOI: 10. $1177 / 0271678 X 15612487$.

[39] S. M. Shams Kazmi, Ehssan Faraji, Mitchell A. Davis, Yu-Yen Huang, Xiaojing J. Zhang, and Andrew K. Dunn. "Flux or speed? Examining speckle contrast imaging of vascular flows." In: Biomed. Opt. Express 6.7 (July 2015), pp. 2588-2608. DOI: 10.1364/BOE.6.002588.

[40] Ashwin B. Parthasarathy, S. M. Shams Kazmi, and Andrew K. Dunn. "Quantitative imaging of ischemic stroke through thinned skull in mice with Multi Exposure Speckle Imaging." In: Biomedical optics express 1.1 (July 2010), pp. 246-259. ISSN: 2156-7085. DOI: 10.1364/BOE.1.000246.

[41] Karel E Y Claes, Henk Hoeksema, Tom Vyncke, Jozef Verbelen, Petra De Coninck, Ignace De Decker, and Stan Monstrey. "Evidence Based Burn Depth Assessment Using Laser-Based Technologies: Where Do We Stand?" In: Journal of Burn Care \& Research 42.3 (Oct. 2020), pp. 513-525. ISSN: 1559-047X. DOI: $10.1093 / \mathrm{jbcr} /$ iraa195.

[42] Henk Hoeksema, Karlien Van de Sijpe, Thiery Tondu, Moustapha Hamdi, Koenraad Van Landuyt, Phillip Blondeel, and Stan Monstrey. "Accuracy of early burn depth assessment by laser Doppler imaging on different days post burn." In: Burns 35.1 (2009), pp. 36-45. ISSN: 0305-4179. DOI: 10.1016/j.burns.2008.08.011.

[43] Robin Mirdell, Simon Farnebo, Folke Sjöberg, and Erik Tesselaar. "Accuracy of laser speckle contrast imaging in the assessment of pediatric scald wounds." In: Burns 44.1 (2018), pp. 90-98. ISSN: 0305-4179. DOI: 10.1016/j.burns.2017.06.010.

[44] Robin Mirdell, Simon Farnebo, Folke Sjöberg, and Erik Tesselaar. "Using blood flow pulsatility to improve the accuracy of laser speckle contrast imaging in the assessment of burns." In: Burns 46.6 (2020), pp. 1398-1406. ISSN: 0305-4179. DOI: $10.1016 / \mathrm{j}$. burns.2020.03.008.

[45] Jun Shu and Gaetano Santulli. "Update on peripheral artery disease: Epidemiology and evidence-based facts." In: Atherosclerosis 275 (2018), pp. 379-381. ISSN: 00219150. DOI: $10.1016 / \mathrm{j}$.atherosclerosis.2018.05.033.

[46] Michael S. Conte, Andrew W. Bradbury, Philippe Kolh, John V. White, Florian Dick, Robert Fitridge, Joseph L. Mills, Jean-Baptiste Ricco, Kalkunte R. Suresh, M. Hassan Murad, Victor Aboyans, Murat Aksoy, Vlad-Adrian Alexandrescu, David Armstrong, Nobuyoshi Azuma, Jill Belch, Michel Bergoeing, Martin Bjorck, Nabil Chakfé, Stephen Cheng, Joseph Dawson, Eike Sebastian Debus, Andrew Dueck, Susan Duval, Hans Henning Eckstein, Roberto Ferraresi, Raghvinder Gambhir, Mauro Gargiulo, Patrick Geraghty, Steve Goode, Bruce Gray, Wei Guo, Prem Chand Gupta, Robert Hinchliffe, Prasad Jetty, Kimihiro Komori, Lawrence Lavery, Wei Liang, Robert Lookstein, Matthew Menard, Sanjay Misra, Tetsuro Miyata, Greg Moneta, Jose Antonio Munoa Prado, Alberto Munoz, Juan Esteban Paolini, Manesh Patel, Frank Pomposelli, Richard Powell, Peter Robless, Lee Rogers, Andres Schanzer, Peter Schneider, Spence Taylor, Melina Vega De Ceniga, Martin Veller, Frank Vermassen, Jinsong Wang, and Shenming Wang. "Global vascular guidelines on the management of chronic limb-threatening ischemia." In: Journal of Vascular Surgery 69.6 (June 2019), 3S-125S.e40. ISSN: 0741-5214. DOI: 10.1016/j.jvs.2019.02.016.

[47] Anne Humeau-Heurtier, Pierre Abraham, and Samir Henni. "Bi-dimensional variational mode decomposition of laser speckle contrast imaging data: A clinical approach to critical limb ischemia?" In: Computers in Biology and Medicine 86 (2017), pp. 107-112. ISSN: 0010-4825. DOI: 10.1016/j.compbiomed.2017.05.012. 
[48] Sotaro Katsui, Yoshinori Inoue, Kimihiro Igari, Takahiro Toyofuku, Toshifumi Kudo, and Hiroyuki Uetake. "Novel assessment tool based on laser speckle contrast imaging to diagnose severe ischemia in the lower limb for patients with peripheral arterial disease." In: Lasers in surgery and medicine 49.7 (Sept. 2017), pp. 645-651. ISSN: 1096-9101. DOI: 10.1002/1sm.22669.

[49] Sotaro Katsui, Yoshinori Inoue, Yohei Yamamoto, Kimihiro Igari, Toshifumi Kudo, and Hiroyuki Uetake. "In Patients with Severe Peripheral Arterial Disease, Revascularization-Induced Improvement in Lower Extremity Ischemia Can Be Detected by Laser Speckle Contrast Imaging of the Fluctuation in Blood Perfusion after Local Heating." In: Annals of Vascular Surgery 48 (2018), pp. 67-74. ISSN: 0890-5096. DOI: 10.1016/j.avsg.2017.09.022.

[50] Shinsuke Kikuchi, Keisuke Miyake, Yuki Tada, Daiki Uchida, Atsuhiro Koya, Yukihiro Saito, Takehiko Ohura, and Nobuyoshi Azuma. "Laser speckle flowgraphy can also be used to show dynamic changes in the blood flow of the skin of the foot after surgical revascularization." In: Vascular 27.3 (June 2019), pp. 242-251. ISSN: 1708-539X. DOI: 10.1177/1708538118810664.

[51] Onno A. Mennes, Jaap J. van Netten, Jeff G. van Baal, Riemer H. J. A. Slart, and Wiendelt Steenbergen. "The Association between Foot and Ulcer Microcirculation Measured with Laser Speckle Contrast Imaging and Healing of Diabetic Foot Ulcers." In: Journal of Clinical Medicine 10.17 (2021). ISSN: 2077-0383. DOI: 10.3390/jcm10173844.

[52] Vittorio Bianco, Pasquale Memmolo, Marco Leo, Silvio Montresor, Cosimo Distante, Melania Paturzo, Pascal Picart, Bahram Javidi, and Pietro Ferraro. "Strategies for reducing speckle noise in digital holography." In: Light: Science $\&$ Applications 7.1 (Aug. 2018), p. 48. ISSN: 2047-7538. DOI: 10.1038/s41377-018-0050-9.

[53] J. W. Goodman. "Some fundamental properties of speckle*." In: J. Opt. Soc. Am. 66.11 (Nov. 1976), pp. 1145-1150. DOI: 10.1364/JOSA.66.001145.

[54] Ingemar Fredriksson and Marcus Larsson. "On the equivalence and differences between laser Doppler flowmetry and laser speckle contrast analysis." In: Journal of Biomedical Optics 21.12 (2016), pp. 1-11. DOI: 10.1117/1.JBO.21.12.126018.

[55] Anne Humeau, Wiendelt Steenbergen, Henrik Nilsson, and Tomas Strömberg. "Laser Doppler perfusion monitoring and imaging: novel approaches." In: Medical E Biological Engineering $\&$ Computing 45.5 (Mar. 2007), p. 421. ISSN: 1741-0444. DOI: $10.1007 /$ s11517-007-0170-5.

[56] Ingemar Fredriksson, Marcus Larsson, and Tomas Strömberg. "Absolute flow velocity components in laser Doppler flowmetry." In: Optical Diagnostics and Sensing VI. Ed. by Gerard L. Coté and Alexander V. Priezzhev. Vol. 6094. International Society for Optics and Photonics. SPIE, 2006, pp. 48-59. DOI: 10.1117/12.659206.

[57] Marcus Larsson. "Influence of optical properties on laser Doppler flowmetry." $\mathrm{PhD}$ thesis. Linköping University, 2004. URL: https : / /www . diva-portal .org/smash/ record.jsf?pid=diva2\%5C\%3A249744\&dswid=-8059.

[58] Sigal Ragol, Itay Remer, Yaron Shoham, Sivan Hazan, Udi Willenz, Igor Sinelnikov, Vladimir Dro11, Lior Rosenberg, and Alberto Bilenca. "Static laser speckle contrast analysis for noninvasive burn diagnosis using a camera-phone imager." In: Journal of Biomedical Optics 20.8 (2015), pp. 1-9. DOI: 10.1117/1. JBO.20.8.086009.

[59] P.-A. Lemieux and D. J. Durian. "Investigating non-Gaussian scattering processes by using nth-order intensity correlation functions." In: J. Opt. Soc. Am. A 16.7 (July 1999), pp. 1651-1664. DOI: 10.1364/JOSAA.16.001651. 
[60] Dilleys Ferreira, Romain Bachelard, William Guerin, Robin Kaiser, and Mathilde Fouché. "Connecting field and intensity correlations: The Siegert relation and how to test it." In: American Journal of Physics 88.10 (2020), pp. 831-837. DOI: 10 . 1119/10.0001630.

[61] Hidemitsu Furukawa and Shunsuke Hirotsu. "Dynamic Light Scattering from Static and Dynamic Fluctuations in Inhomogeneous Media." In: Journal of the Physical Society of Japan 71.12 (2002), pp. 2873-2880. DOI: 10.1143/JPSJ.71.2873.

[62] L. Cohen. "The generalization of the Wiener-Khinchin theorem." In: Proceedings of the 1998 IEEE International Conference on Acoustics, Speech and Signal Processing, ICASSP '98 (Cat. No.98CH36181). Vol. 3. 1998, 1577-1580 vol.3. DOI: 10.1109/ICASSP. 1998.681753.

[63] R. Bandyopadhyay, A. S. Gittings, S. S. Suh, P. K. Dixon, and D. J. Durian. "Speckle-visibility spectroscopy: A tool to study time-varying dynamics." In: Review of Scientific Instruments 76.9 (2005), p. 093110. DOI: 10.1063/1.2037987.

[64] Pavel Zakharov, Andreas Charles Völker, Alfred Buck, Bruno Weber, and Frank Scheffold. "Quantitative modeling of laser speckle imaging." In: Optics letters 3123 (2006), pp. 3465-7.

[65] David A. Boas and Andrew K. Dunn. "Laser speckle contrast imaging in biomedical optics." In: Journal of Biomedical Optics 15.1 (2010), pp. 1-12. DOI: 10.1117/1. 3285504.

[66] Donald D. Duncan, Sean J. Kirkpatrick, and Ruikang K. Wang. "Statistics of local speckle contrast." In: J. Opt. Soc. Am. A 25.1 (Jan. 2008), pp. 9-15. DOI: 10.1364/ JOSAA. 25.000009.

[67] J. C. Ramirez-San-Juan, E. Mendez-Aguilar, N. Salazar-Hermenegildo, A. FuentesGarcia, R. Ramos-Garcia, and B. Choi. "Effects of speckle/pixel size ratio on temporal and spatial speckle-contrast analysis of dynamic scattering systems: Implications for measurements of blood-flow dynamics." In: Biomed. Opt. Express 4.10 (Oct. 2013), pp. 1883-1889. DOI: 10.1364/BOE.4.001883.

[68] Sean J. Kirkpatrick, Donald D. Duncan, and Elaine M. Wells-Gray. "Detrimental effects of speckle-pixel size matching in laser speckle contrast imaging." In: Opt. Lett. 33.24 (Dec. 2008), pp. 2886-2888. DOI: 10.1364/OL.33.002886.

[69] Chenge Wang, Zili Cao, Xin Jin, Weihao Lin, Yang Zheng, Bixin Zeng, and M. Xu. "Robust quantitative single-exposure laser speckle imaging with true flow speckle contrast in the temporal and spatial domains." In: Biomed. Opt. Express 10.8 (Aug. 2019), pp. 4097-4114. DOI: 10.1364/BOE.10.004097.

[70] Claudia P. Valdes, Hari M. Varma, Anna K. Kristoffersen, Tanja Dragojevic, Joseph P. Culver, and Turgut Durduran. "Speckle contrast optical spectroscopy, a noninvasive, diffuse optical method for measuring microvascular blood flow in tissue." In: Biomed. Opt. Express 5.8 (Aug. 2014), pp. 2769-2784. DOI: 10.1364/BOE. 5 . 002769 .

[71] Matthijs Draijer, Erwin Hondebrink, Ton van Leeuwen, and Wiendelt Steenbergen. "Review of laser speckle contrast techniques for visualizing tissue perfusion." In: Lasers in medical science 24.4 (July 2009), pp. 639-651. ISSN: 1435-604X. DOI: 10.1007/s10103-008-0626-3.

[72] Donald D. Duncan, Sean J. Kirkpatrick, and James C. Gladish. "What is the proper statistical model for laser speckle flowmetry?" In: Complex Dynamics and Fluctuations in Biomedical Photonics V. Ed. by Valery V. Tuchin and Lihong V. Wang. Vol. 6855. International Society for Optics and Photonics. SPIE, 2008, pp. 9-15. DOI: $10.1117 / 12.760515$.

[73] Erf. URL: https://mathworld.wolfram.com/Erf.html. 
[74] G.J. Richards and J.D. Briers. "Laser speckle contrast analysis (LASCA): a technique for measuring capillary blood flow using the first order statistics of laser speckle patterns." In: IEE Colloquium on Biomedical Applications of Photonics (Digest No. 1997/124). 1997, pp. 11/1-11/6. DOI: 10.1049/ic: 19970687.

[75] Julio C Ramirez-San-Juan, Ruben Ramos-Garcia, Ileana Guizar-Iturbide, Gabriel Martinez-Niconoff, and Bernard Choi. "Impact of velocity distribution assumption on simplified laser speckle imaging equation." In: Opt. Express 16.5 (Mar. 2008), pp. 3197-3203. DOI: 10.1364/OE.16.003197.

[76] Shuai Yuan, Anna Devor, David A. Boas, and Andrew K. Dunn. "Determination of optimal exposure time for imaging of blood flow changes with laser speckle contrast imaging." In: Appl. Opt. 44.10 (Apr. 2005), pp. 1823-1830. DOI: 10.1364/A0.44 . 001823.

[77] Pedro G Vaz, Anne Humeau-Heurtier, Edite Figueiras, Carlos Correia, and João Cardoso. "Effect of static scatterers in laser speckle contrast imaging: an experimental study on correlation and contrast." In: Physics in Medicine 8 Biology 63.1 (Dec. 2017), p. 015024. DOI: 10.1088/1361-6560/aa9f3a.

[78] KENNETH LEVENBERG. "A METHOD FOR THE SOLUTION OF CERTAIN NON-LINEAR PROBLEMS IN LEAST SQUARES." In: Quarterly of Applied Mathematics 2.2 (1944), pp. 164-168. ISSN: 0033569X, 15524485. URL: http:// www.jstor.org/stable/43633451.

[79] Donald W. Marquardt. "An Algorithm for Least-Squares Estimation of Nonlinear Parameters." In: Journal of the Society for Industrial and Applied Mathematics 11.2 (1963), pp. 431-441. ISSN: 03684245. URL: http://www.jstor.org/stable/2098941.

[80] David Briers, Donald D. Duncan, Evan R. Hirst, Sean J. Kirkpatrick, Marcus Larsson, Wiendelt Steenbergen, Tomas Stromberg, and Oliver B. Thompson. "Laser speckle contrast imaging: theoretical and practical limitations." In: Journal of Biomedical Optics 18.6 (2013), pp. 1-10. DOI: 10.1117/1.JBO.18.6.066018.

[81] Mitchell A. Davis, S. M. Shams Kazmi, and Andrew K. Dunn. "Imaging depth and multiple scattering in laser speckle contrast imaging." In: Journal of biomedical optics 19.8 (Aug. 2014), pp. 086001-086001. ISSN: 1560-2281. DOI: 10.1117/1. JBO. 19.8 .086001 .

[82] Ingemar Fredriksson. "Quantitative Laser Doppler Flowmetry." PhD thesis. Linköping University, 2009. URL: http : / / urn . kb. se / resolve? urn=urn : nbn : se:liu:diva-19947.

[83] Ingemar Fredriksson, Oleg Burdakov, Marcus Larsson, and Tomas Strömberg. "Inverse Monte Carlo in a multilayered tissue model: merging diffuse reflectance spectroscopy and laser Doppler flowmetry." In: Journal of Biomedical Optics 18.12 (2013), pp. 1-15. DOI: 10.1117/1.JBO.18.12.127004.

[84] Ingemar Fredriksson, Marcus Larsson, and Tomas Strömberg. "Machine learning for direct oxygen saturation and hemoglobin concentration assessment using diffuse reflectance spectroscopy." In: Journal of Biomedical Optics 25.11 (2020), pp. 1-16. DOI: $10.1117 / 1 . J B 0.25 .11 .112905$.

[85] Ingemar Fredriksson, Marcus Larsson, and Tomas Strömberg. "Inverse Monte Carlo method in a multilayered tissue model for diffuse reflectance spectroscopy." In: Journal of Biomedical Optics 17.4 (2012), pp. 1-13. DOI: 10.1117/1. JBO.17.4. 047004

[86] Ingemar Fredriksson, Marcus Larsson, and Tomas Strömberg. "Optical microcirculatory skin model: assessed by Monte Carlo simulations paired with in vivo laser Doppler flowmetry." In: Journal of Biomedical Optics 13.1 (2008), pp. 1-12. DOI: $10.1117 / 1.2854691$. 
[87] Ingemar Fredriksson, Marcus Larsson, and Tomas Strömberg. "Model-based quantitative laser Doppler flowmetry in skin." In: Journal of Biomedical Optics 15.5 (2010), pp. 1-12. DOI: 10.1117/1.3484746.

[88] Ingemar Fredriksson and Marcus Larsson. "Vessel packaging effect in laser speckle contrast imaging and laser Doppler imaging." In: Journal of Biomedical Optics 22.10 (2017), pp. 1-7. DOI: 10.1117/1. JBO.22.10.106005.

[89] G. Bergström, G. Berglund, A. Blomberg, J. Brandberg, G. Engström, J. Engvall, M. Eriksson, U. de Faire, A. Flinck, M. G. Hansson, B. Hedblad, O. Hjelmgren, C. Janson, T. Jernberg, Å. Johnsson, L. Johansson, L. Lind, C.-G. Löfdahl, O. Melander, C. J. Östgren, A. Persson, M. Persson, A. Sandström, C. Schmidt, S. Söderberg, J. Sundström, K. Toren, A. Waldenström, H. Wedel, J. Vikgren, B. Fagerberg, and A. Rosengren. "The Swedish CArdioPulmonary BioImage Study: objectives and design." In: Journal of Internal Medicine 278.6 (2015), pp. 645-659. DOI: https://doi.org/10.1111/joim.12384.

[90] Hanna Jonasson, Sara Bergstrand, Ingemar Fredriksson, Marcus Larsson, Carl Johan Östgren, and Tomas Strömberg. "Normative data and the influence of age and sex on microcirculatory function in a middle-aged cohort: results from the SCAPIS study." In: American journal of physiology. Heart and circulatory physiology 318.4 (Apr. 2020), H908-H915. ISSN: 1522-1539. DOI: 10.1152/ajpheart.00668.2019.

[91] Hanna Jonasson, Ingemar Fredriksson, Marcus Larsson, and Tomas Strömberg. "Validation of speed-resolved laser Doppler perfusion in a multimodal optical system using a blood-flow phantom." In: Journal of Biomedical Optics 24.9 (2019), pp. 1-8. DOI: $10.1117 / 1$.JBO 24.9.095002.

[92] Evgeny Zherebtsov, Viktor Dremin, Alexey Popov, Alexander Doronin, Daria Kurakina, Mikhail Kirillin, Igor Meglinski, and Alexander Bykov. "Hyperspectral imaging of human skin aided by artificial neural networks." In: Biomed. Opt. Express 10.7 (July 2019), pp. 3545-3559. DOI: 10.1364/BOE.10.003545.

[93] Tony F. Chan, Gene H. Golub, and Randall J. LeVeque. "Algorithms for Computing the Sample Variance: Analysis and Recommendations." In: The American Statistician 37.3 (1983), pp. 242-247. ISSN: 00031305. URL: http://www.jstor.org/ stable/2683386.

[94] Maria Ewerlöf, Göran E. Salerud, Tomas Strömberg, and Marcus Larsson. "Estimation of skin microcirculatory hemoglobin oxygen saturation and red blood cell tissue fraction using a multispectral snapshot imaging system: a validation study." In: Journal of Biomedical Optics 26.2 (2021), pp. 1-15. DOI: 10.1117/1. JBO.26.2. 026002. URL: https://doi.org/10.1117/1.JBO.26.2.026002.

[95] Chang Liu, Kivilcim Kiliç, Sefik Evren Erdener, David A. Boas, and Dmitry D. Postnov. "Choosing a model for laser speckle contrast imaging." In: Biomed. Opt. Express 12.6 (June 2021), pp. 3571-3583. DOI: 10.1364/BOE. 426521.

[96] Ingemar Fredriksson, Marcus Larsson, Fredrik H. Nyström, Toste Länne, Carl J. Östgren, and Tomas Strömberg. "Reduced Arteriovenous Shunting Capacity After Local Heating and Redistribution of Baseline Skin Blood Flow in Type 2 Diabetes Assessed With Velocity-Resolved Quantitative Laser Doppler Flowmetry." In: Diabetes 59.7 (2010), pp. 1578-1584. ISSN: 0012-1797. DOI: 10.2337/db10-0080.

[97] S Wojtkiewicz, E Wojcik-Sosnowska, M Jasik, R Maniewski, W Karnafel, and A Liebert. "Assessment of speed distribution of red blood cells in the microvascular network in healthy volunteers and type 1 diabetes using laser Doppler spectra decomposition." In: 35.2 (Jan. 2014), pp. 283-295. DOI: 10.1088/0967-3334/35/2/ 283. 
[98] Hanna Jonasson, Sara Bergstrand, Fredrik H Nystrom, Toste Länne, Carl Johan Östgren, Niclas Bjarnegård, Ingemar Fredriksson, Marcus Larsson, and Tomas Strömberg. "Skin microvascular endothelial dysfunction is associated with type 2 diabetes independently of microalbuminuria and arterial stiffness." In: Diabetes and Vascular Disease Research 14.4 (2017), pp. 363-371. DOI: 10.1177 / 1479164117707706.

[99] Marc G. Jeschke, Margriet E. van Baar, Mashkoor A. Choudhry, Kevin K. Chung, Nicole S. Gibran, and Sarvesh Logsetty. "Burn injury." In: Nature Reviews Disease Primers 6.1 (Feb. 2020), p. 11. ISSN: 2056-676X. DOI: 10.1038/s41572-020-0145-5.

[100] Marco Domenico Cirillo, Robin Mirdell, Folke Sjöberg, and Tuan D Pham. "TimeIndependent Prediction of Burn Depth Using Deep Convolutional Neural Networks." In: Journal of Burn Care ES Research 40.6 (June 2019), pp. 857-863. ISSN: 1559-047X. DOI: 10.1093/jbcr/irz103.

[101] Chong Jiao, Kehua Su, Weiguo Xie, and Ziqing Ye. "Burn image segmentation based on Mask Regions with Convolutional Neural Network deep learning framework: more accurate and more convenient." In: Burns \& trauma 7 (Feb. 2019), pp. 6-6. ISSN: 2321-3868. DOI: 10.1186/s41038-018-0137-9.

[102] Marco Domenico Cirillo, Robin Mirdell, Folke Sjöberg, and Tuan D. Pham. "Improving burn depth assessment for pediatric scalds by AI based on semantic segmentation of polarized light photography images." In: Burns 47.7 (2021), pp. 15861593. ISSN: 0305-4179. DOI: 10.1016/j.burns.2021.01.011.

[103] A. Stefanovska, M. Bracic, and H.D. Kvernmo. "Wavelet analysis of oscillations in the peripheral blood circulation measured by laser Doppler technique." In: IEEE Transactions on Biomedical Engineering 46.10 (1999), pp. 1230-1239. DOI: 10 . 1109/10.790500.

[104] Per Kvandal, Svein Aslak Landsverk, Alan Bernjak, Aneta Stefanovska, Hebe Désirée Kvernmo, and Knut Arvid Kirkebøen. "Low-frequency oscillations of the laser Doppler perfusion signal in human skin." In: Microvascular Research 72.3 (2006), pp. 120-127. ISSN: 0026-2862. DOI: 10.1016/j.mvr.2006.05.006.

[105] Anne Humeau, Audrey Koïtka, Pierre Abraham, Jean-Louis Saumet, and JeanPierre L'Huillier. "Time-frequency analysis of laser Doppler flowmetry signals recorded in response to a progressive pressure applied locally on anaesthetized healthy rats." In: 49.5 (Feb. 2004), pp. 843-857. DOI: 10.1088/0031-9155/49/5/014.

[106] Anne Humeau, Audrey Koïtka, Pierre Abraham, Jean-Louis Saumet, and JeanPierre L'Huillier. "Spectral components of laser Doppler flowmetry signals recorded in healthy and type 1 diabetic subjects at rest and during a local and progressive cutaneous pressure application: scalogram analyses." In: 49.17 (Aug. 2004), pp. 39573970. DOI: $10.1088 / 0031-9155 / 49 / 17 / 009$.

[107] Ingemar Fredriksson, Marcus Larsson, Tomas Strömberg, and Fredrik Iredahl. "Vasomotion analysis of speed resolved perfusion, oxygen saturation, red blood cell tissue fraction, and vessel diameter: Novel microvascular perspectives." In: Skin Research and Technology n/a.n/a (Nov. 2021). DOI: 10.1111/srt.13106.

[108] Lipei Song and Daniel S. Elson. "Effect of signal intensity and camera quantization on laser speckle contrast analysis." In: Biomedical optics express 4.1 (Jan. 2013), pp. 89-104. ISSN: 2156-7085. DOI: 10.1364/BOE.4.000089.

[109] Jianjun Qiu, Yangyang Li, Qin Huang, Yang Wang, and Pengcheng Li. "Correcting speckle contrast at small speckle size to enhance signal to noise ratio for laser speckle contrast imaging." In: Opt. Express 21.23 (Nov. 2013), pp. 28902-28913. DOI: 10.1364/OE.21.028902. 
[110] Oliver Thompson, Michael Andrews, and Evan Hirst. "Correction for spatial averaging in laser speckle contrast analysis." In: Biomed. Opt. Express 2.4 (May 2011), pp. 1021-1029. DOI: 10.1364/BOE.2.001021.

[111] W. James Tom, Adrien Ponticorvo, and Andrew K. Dunn. "Efficient Processing of Laser Speckle Contrast Images." In: IEEE Transactions on Medical Imaging 27.12 (2008), pp. 1728-1738. DOI: 10.1109/TMI.2008.925081.

[112] Md. Zahangir Alom, Tarek M. Taha, Christopher Yakopcic, Stefan Westberg, Paheding Sidike, Mst Shamima Nasrin, Brian C. Van Essen, Abdul A. S. Awwal, and Vijayan K. Asari. "The History Began from AlexNet: A Comprehensive Survey on Deep Learning Approaches." In: CoRR abs/1803.01164 (2018). URL: http: //arxiv.org/abs/1803.01164.

[113] Alex Krizhevsky, Ilya Sutskever, and Geoffrey E Hinton. "ImageNet Classification with Deep Convolutional Neural Networks." In: Advances in Neural Information Processing Systems. Ed. by F. Pereira, C. J. C. Burges, L. Bottou, and K. Q. Weinberger. Vol. 25. Curran Associates, Inc., 2012. URL: https : //proceedings . neurips.cc/paper/2012/file/c399862d3b9d6b76c8436e924a68c45b-Paper.pdf.

[114] Kaiming He, Xiangyu Zhang, Shaoqing Ren, and Jian Sun. "Deep Residual Learning for Image Recognition." In: CoRR abs/1512.03385 (2015). URL: http://arxiv . org/abs/1512.03385.

[115] Tom B. Brown, Benjamin Mann, Nick Ryder, Melanie Subbiah, Jared Kaplan, Prafulla Dhariwal, Arvind Neelakantan, Pranav Shyam, Girish Sastry, Amanda Askell, Sandhini Agarwal, Ariel Herbert-Voss, Gretchen Krueger, Tom Henighan, Rewon Child, Aditya Ramesh, Daniel M. Ziegler, Jeffrey Wu, Clemens Winter, Christopher Hesse, 3k Chen, Eric Sigler, Mateusz Litwin, Scott Gray, Benjamin Chess, Jack Clark, Christopher Berner, Sam McCandlish, Alec Radford, Ilya Sutskever, and Dario Amodei. "Language Models are Few-Shot Learners." In: CoRR abs/2005.14165 (2020). URL: https://arxiv.org/abs/2005.14165.

[116] Cynthia Rudin. "Stop explaining black box machine learning models for high stakes 12isions and use interpretable models instead." In: Nature Machine Intelligence 1.5 (May 2019), pp. 206-215. ISSN: 2522-5839. DOI: 10.1038/s42256-019-0048-x.

[117] S. M. Shams Kazmi, Satyajit Balial, and Andrew K. Dunn. "Optimization of camera exposure durations for multi-exposure speckle imaging of the microcirculation." In: Biomedical optics express 5.7 (June 2014), pp. 2157-2171. ISSN: 2156-7085. DOI: 10.1364/BOE.5.002157.

[118] Ferenc Domoki, Dániel Zölei, Orsolya Oláh, Valéria Tóth-Szűki, Béla Hopp, Ferenc Bari, and Tomi Smausz. "Evaluation of laser-speckle contrast image analysis techniques in the cortical microcirculation of piglets." In: Microvascular Research 83.3 (2012), pp. 311-317. ISSN: 0026-2862. DOI: 10.1016/j.mvr.2012.01.003.

[119] Syed Mohammad Shams Kazmi, Ashwin B. Parthasarthy, Nelly E. Song, Theresa A. Jones, and Andrew K. Dunn. "Chronic imaging of cortical blood flow using MultiExposure Speckle Imaging." In: Journal of cerebral blood flow and metabolism : official journal of the International Society of Cerebral Blood Flow and Metabolism 33.6 (June 2013), pp. 798-808. ISSN: 1559-7016. DOI: 10.1038/jcbfm.2013.57.

[120] Ashwin B. Parthasarathy, Weon Gyu Shin, X. J. Zhang, and A. K. Dunn. "Laser speckle contrast imaging of flow in a microfluidic device." In: Biomedical Applications of Light Scattering. Ed. by Adam Wax and Vadim Backman. Vol. 6446. International Society for Optics and Photonics. SPIE, 2007, pp. 19-29. DOI: 10 . $1117 / 12.700572$

[121] Zhe Chen, Mengyang Liu, Michael Minneman, Laurin Ginner, Erich Hoover, Harald Sattmann, Marco Bonesi, Wolfgang Drexler, and Rainer A. Leitgeb. "Phase-stable swept source OCT angiography in human skin using an akinetic source." In: Biomed. Opt. Express 7.8 (Aug. 2016), pp. 3032-3048. DOI: 10.1364/BOE.7.003032. 
[122] Tyler B. Rice, Soren D. Konecky, Amaan Mazhar, David J. Cuccia, Anthony J. Durkin, Bernard Choi, and Bruce J. Tromberg. "Quantitative determination of dynamical properties using coherent spatial frequency domain imaging." In: J. Opt. Soc. Am. A 28.10 (Oct. 2011), pp. 2108-2114. DOI: 10.1364/JOSAA.28.002108.

[123] Tyler B. Rice, Elliott Kwan, Carole K. Hayakawa, Anthony J. Durkin, Bernard Choi, and Bruce J. Tromberg. "Quantitative, depth-resolved determination of particle motion using multi-exposure, spatial frequency domain laser speckle imaging." In: Biomedical optics express 4.12 (Nov. 2013), pp. 2880-2892. ISSN: 2156-7085. DOI: 10.1364/BOE.4.002880.

[124] Michael T. Ghijsen, Griffin R. Lentsch, Sylvain Gioux, Matthew Brenner M.D., Anthony J. Durkin, Bernard Choi, and Bruce J. Tromberg. "Quantitative real-time optical imaging of the tissue metabolic rate of oxygen consumption." In: Journal of Biomedical Optics 23.3 (2018), pp. 1-12. DOI: 10.1117/1.JBO.23.3.036013. 



\section{Papers}

The papers associated with this thesis have been removed for copyright reasons. For more details about these see:

https://doi.org/10.3384/9789179291068 



\section{FACULTY OF SCIENCE AND ENGINEERING}

Linköping Studies in Science and Technology, Dissertation No. 2187, 2021 Department of Biomedical Engineering

Linköping University

SE-58183 Linköping, Sweden

www.liu.se 